\title{
Parametric Studies on UAV Flying Qualities
}

ANA LESIARIO

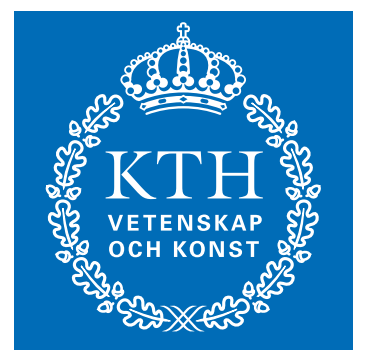

KTH Electrical Engineering

Masters' Degree Project

Stockholm, Sweden Oct 2009 


\title{
Academia da Força Aérea Royal Institute of Technology
}

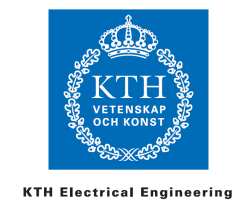

\section{Parametric Studies on UAV Flying Qualities}

\author{
Ana Sofia Andrês dos Reis Lesiário
}

Master's Thesis in Aeronautical Engineering

Examiner: Karl Henrik Johansson (Professor)

Supervisor: Martin Hagström (Project Manager)

October 2009 


\section{Abstract}

When developing an aircraft, one of several important aspects is to predict and properly design the dynamic behaviour of the aircraft. This holds for manned aircraft as well as for UAVs. The optimal dynamic behaviour for an aircraft depends on the mission or purpose: for a certain use an aircraft should be agile, other may require a more stable one. In aeronautics, the properties that describe the aircraft efficacy with respect to some task are known as flying qualities, and our goal is to study their dependence on some design parameters. As a test model we use an existing UAV. After deriving its 6-DOF dynamic model and assessing its baseline characteristics, we perform parametric studies. The strategy followed is divided in two steps: the first consists on analyzing flying qualities sensitivity to changes in model parameters. The second step studies how specific design changes affect model parameters. Because the first step only depends on the dynamic model form, we verify, by testing two other different aircrafts, that conclusions drawn from this step are valid to other configurations. Finally we show how results from parametric studies can be used to improve the UAV flying qualities regarding a certain mission, through the introduction of slight modifications on baseline design.

Key-Words: UAV, Flying Qualities, Design Parameters, Dynamic Model 


\section{Acknowledgements}

First, I would like to express my sincere gratitude to my supervisor, Dr. Martin Hagström, from FOI, for all his support and many working hours spent with this project. Despite his busy life, Martin has always been available to help me solve any arising question or problem, even on weekends. Although he says that I am very independent doing my work, I would never be able to go through with it without his assistance and advice.

I would also like to thank Dr. Olivier Amoignon, from FOI, for doing all CFD simulations and for the discussions we have had on this subject, from which I learned a lot. CFD is not the focus of this project but it is an essential part of it, which is very time demanding. Dr. Olivier's help has been crucial.

My thanks also go to Dr. Fredrik Berefelt, for his revision and advice.

From the Air Force Academy, I would like to thank Lt. Colonel José Morgado and Major Maria Madruga and for inviting me for PITVANT project, on UAV development, and for giving me the amazing opportunity to work in Stockholm, in such prestigious institutions, KTH and FOI, and to learn from the best. Lt Luís Félix and Lt João Caetano also have played important roles in this project and I would like to show my appreciation for their help.

Dr. João Tasso, from Porto University, also deserves my gratitude for the time spent clarifying some questions and for his valuable suggestions.

Last but most important, I would like to thank my family and Hugo Jorge for their unconditional love, patience and support, which helped me carry out this challenging task away from home, in a new home, and for making me the person I am. 


\section{Contents}

1 Introduction $\quad 1$

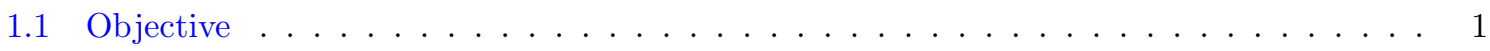

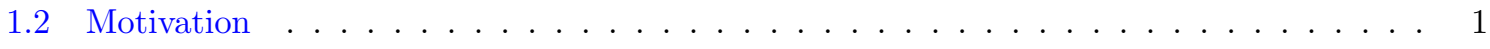

1.3 Problem Statement . . . . . . . . . . . . . . . . . . . . 2

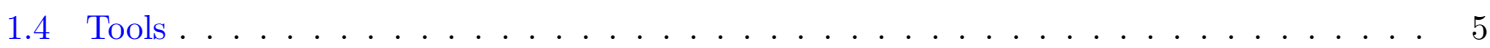

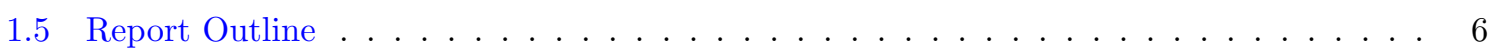

2 Dynamic Model of an Aircraft $\quad 9$

2.1 Dynamic Model Derivation . . . . . . . . . . . . . . . . . . . . . 9

2.1.1 The Equations Collected . . . . . . . . . . . . . . . . 13

2.1.2 Forces and Moments . . . . . . . . . . . . . . . . . . . . 14

2.1.2.1 Gravitational Force ...................... 14

2.1.2.2 Thrust Force and Moment . . . . . . . . . . . . . . . . . 15

2.1.3 Aerodynamic Model . . . . . . . . . . . . . . . . . . . . 15

2.1.3.1 Aerodynamic Model Derivation . . . . . . . . . . . . 16

2.1.3.2 Data Gathering ...................... 18

2.2 Atmospheric Model . . . . . . . . . . . . . . . . . . . . . . . 19

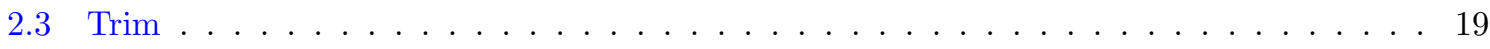

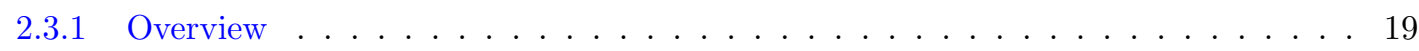

2.3.2 Trim Conditions .......................... 20

2.3.3 Trim Algorithm . . . . . . . . . . . . . . . . . . . . 21

2.4 Linearisation . . . . . . . . . . . . . . . . . . . . . . 21

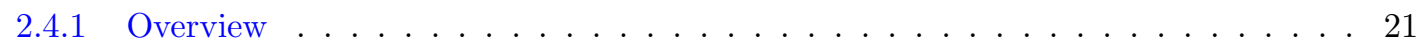

2.4.2 Small Disturbances Equations . . . . . . . . . . . . . . . 23

2.4.2.1 Longitudinal Equations of Motion . . . . . . . . . . . . . . . . 24

2.4.2.2 Lateral Equations of Motion . . . . . . . . . . . . . . 25

2.4 .3 Linearisation Algorithm . . . . . . . . . . . . . . . . . 26 
3 Flying Qualities $r$

3.1 General Overview . . . . . . . . . . . . . . . . . . . . . 29

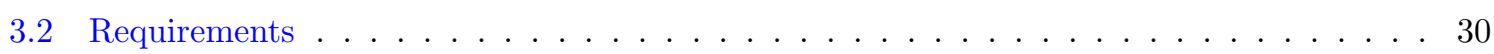

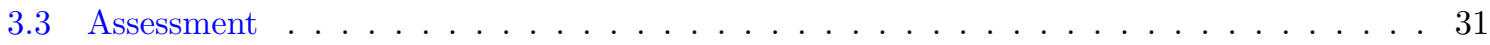

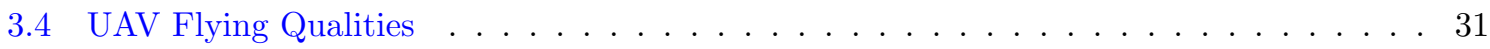

3.5 Choice of Flying Qualities for Analysis . . . . . . . . . . . . . . . . . . 32

3.5.1 Longitudinal Flying Qualities ....................... 33

3.5.2 Lateral Flying Qualities . . . . . . . . . . . . . . . . . . 35

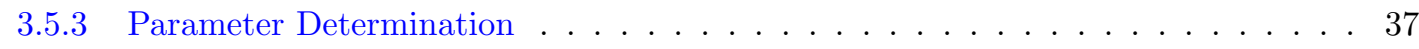

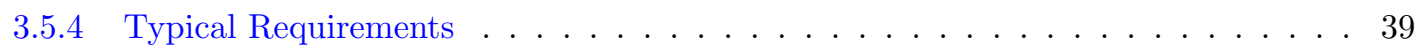

4 ANTEX-M X03 Flying Qualities $\quad 41$

4.1 Dynamic Model . . . . . . . . . . . . . . . . . . . . . . . . . 41

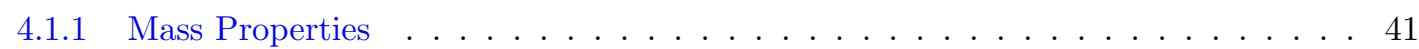

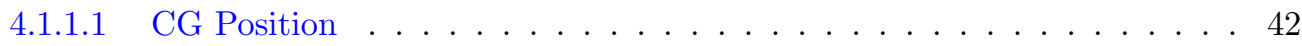

4.1 .1 .2 Inertia Matrix . . . . . . . . . . . . . . . 43

4.1.2 Aerodynamic Model . . . . . . . . . . . . . . . . . . 45

4.1.2.1 Final Aerodynamic Model . . . . . . . . . . . . . . . 47

4.1.2.2 Some Important Results . . . . . . . . . . . . . . . . . . . . 48

4.1 .3 Propulsion System . . . . . . . . . . . . . . . . . . . . . . 49

4.2 Flying Qualities Assessment . . . . . . . . . . . . . . . . . . . . . . 49

4.2.1 Static Characteristics . . . . . . . . . . . . . . . . 49

4.2 .2 Dynamic Characteristics . . . . . . . . . . . . . . . 51

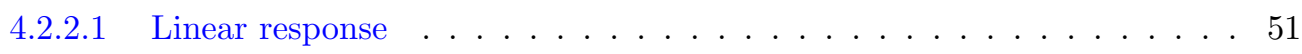

4.2 .3 Nonlinear Response .......................... 59

4.2.4 Dynamic Flying Qualities Collected . . . . . . . . . . . . . . . . 65

4.3 Qualitative Analysis of Flying Qualities . . . . . . . . . . . . . . . . 66

4.4 Effect of Spinning Rotors . . . . . . . . . . . . . . . . . . . . . . 67

5 Parametric Studies $\quad 69$

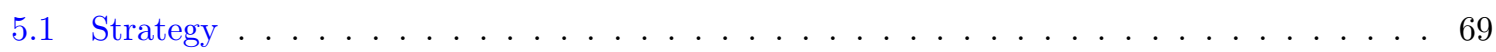

5.2 Choice of Design Parameters . . . . . . . . . . . . . . . . . . . . 70

5.3 Sensitivity Analysis . . . . . . . . . . . . . . . . . . . . 70

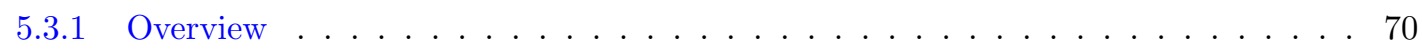

5.3.2 Flying Qualities Sensitivity to Model Parameters . . . . . . . . . . . . . . . 71

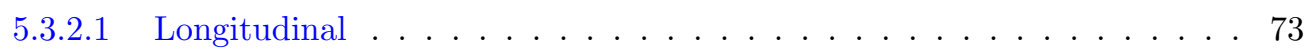

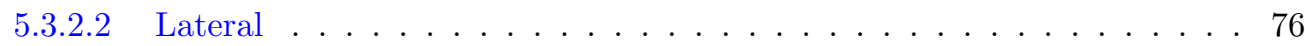

5.3.3 Mass Properties Sensitivity to Design Parameters . . . . . . . . . . . . . 77 
5.3.4 Aerodynamic Characteristics Sensitivity to Design Parameters . . . . . . . . . 80

5.4 Effect of Design Parameters on Flying Qualities . . . . . . . . . . . . . . . 83

5.5 Applicability to Other Aircraft . . . . . . . . . . . . . . . . . . 86

6 Improving ANTEX-M X03 Design 91

6.1 Studying a New Design _ . . . . . . . . . . . . . . . . . . . . . . 91

6.2 Problem Definition . . . . . . . . . . . . . . . . . . . . . . . . . 92

6.2 .1 Mission . . . . . . . . . . . . . . . . . . . . . 92

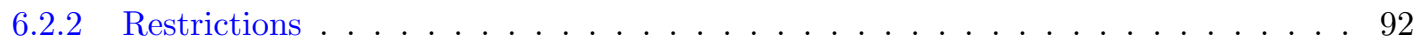

6.3 Analysis of a Design Option $\ldots \ldots \ldots \ldots \ldots \ldots \ldots \ldots \ldots \ldots$

6.3.1 Design Modifications . . . . . . . . . . . . . . . . . . . . . . . 93

6.3.2 Results . . . . . . . . . . . . . . . . . . . . . . . 93

7 Conclusions and Future Work $\quad 97$

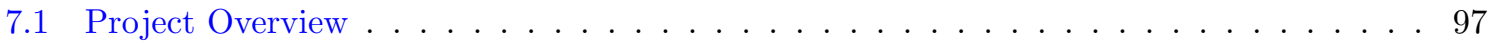

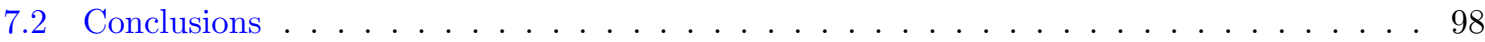

7.3 Future Work . . . . . . . . . . . . . . . . . . . . . . . . . . . . . 99

A ANTEX-X03 Existing Data 101

A.1 Flight Envelope . . . . . . . . . . . . . . . . . . . . . . . . . . . . 101

A.2 Aerodynamic Characteristics . . . . . . . . . . . . . . . . . . . . . . . . . 102

A.2.1 Assumptions . . . . . . . . . . . . . . . . . . . . . . . . . . . . . 102

A.2.2 Summary of Relevant Flight Conditions . . . . . . . . . . . . . . . . . . 103

A.2.3 Tests Performed . . . . . . . . . . . . . . . . . . . . . . . . 103

A.2.4 Additional Remarks . . . . . . . . . . . . . . . . . . . . . . . . . . . . 104

A.2.4.1 Wind Tunnel and CFD Results Comparison . . . . . . . . . . . . . . 104

A.2.4.2 Stability Derivatives . . . . . . . . . . . . . . . 105

A.3 Center of Gravity (CG) Position an Inertia Tensor . . . . . . . . . . . . . . . 105

A.4 Open Loop Stability . . . . . . . . . . . . . . . . . . . . . . . . . . . 105

A.5 Existing Files . . . . . . . . . . . . . . . . . . . . . . . 106

$\begin{array}{ll}\text { Appendices } & 101\end{array}$

B Methods for Estimating Aerodynamic Derivatives $\quad 107$

B.1 Sign Convention . . . . . . . . . . . . . . . . . . . . . . . . 107

B.2 Aircraft Controls . . . . . . . . . . . . . . . . . . . . . . . . . . . . . . . 108

B.3 Geometric Parameters Definition . . . . . . . . . . . . . . . . . . . . . . . . 108

B.4 Longitudinal Derivatives . . . . . . . . . . . . . . . . . . . . . . . . . 110

B.5 Lateral Derivatives . . . . . . . . . . . . . . . . . . . . . . . . . . . . . . 112 
B.5.1 Control Derivatives . . . . . . . . . . . . . . . . . . 113

B.6 Dimensional Derivatives . . . . . . . . . . . . . . . . . . . . . . 114

$\begin{array}{lr}C \text { First and Second Order Systems } & 117\end{array}$

C.1 First Order Systems . . . . . . . . . . . . . . . . . . . . 117

C.2 Second Order System _. . . . . . . . . . . . . . . . . . . . 117

D Moments and Products of Inertia of Regular Volumes 121

E Sensitivity Analysis for Different Aircraft $\quad 125$

E.1 Longitudinal Flying Qualities . . . . . . . . . . . . . . . . . . . . . . . 125

E.2 Lateral Flying Qualities . . . . . . . . . . . . . . . . . . . . . . 125

$\begin{array}{lrr}\text { F Effect of Design on Model Parameters } & 129\end{array}$

Bibliography 133 


\section{List of Figures}

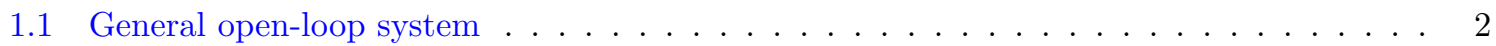

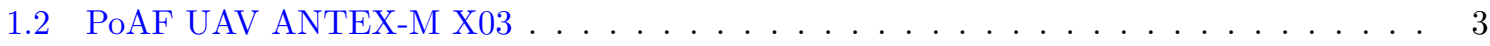

1.3 Main components of an aircraft 6 -DOF dynamic model . . . . . . . . . . . . . 4

1.4 Strategy for parametric studies $\ldots \ldots \ldots \ldots \ldots \ldots \ldots \ldots$

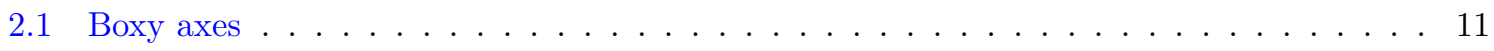

2.2 Longitudinal poles for a conventional aircraft $\ldots \ldots \ldots \ldots \ldots \ldots \ldots \ldots$

2.3 Lateral poles for a conventional aircraft . . . . . . . . . . . . . . . . . . . 26

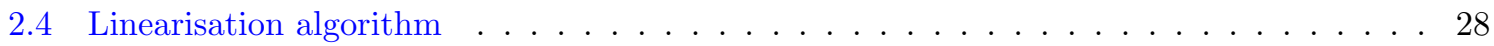

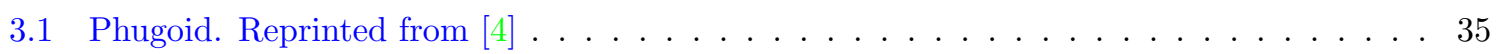

3.2 Short Period Reprinted from $[4] \ldots \ldots \ldots \ldots \ldots \ldots \ldots$

3.3 Roll. Reprinted from $[4] \ldots \ldots \ldots \ldots \ldots \ldots \ldots \ldots$

3.4 Spiral. Reprinted from $[4] \ldots \ldots \ldots \ldots \ldots \ldots \ldots$

3.5 Dutch Roll. Reprinted from $[4] \ldots \ldots \ldots \ldots$. . . . . . . . . . . . . . . . .

4.1 SolidWorks model used for inertia matrix computation . . . . . . . . . . . . . . . 44

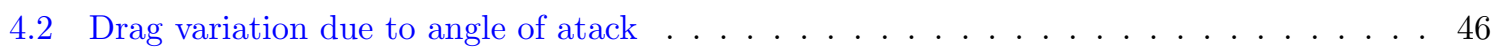

4.3 Drag variation due to elevator deflection . . . . . . . . . . . . . . . 46

4.4 Longitudinal static characteristics in straight level flight . . . . . . . . . . . . . . . 50

4.5 Longitudinal static characteristics in pull-up/push-over . . . . . . . . . . . . 51

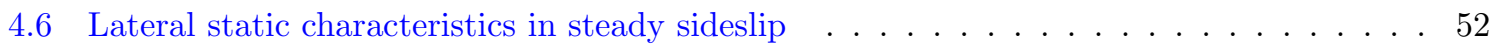

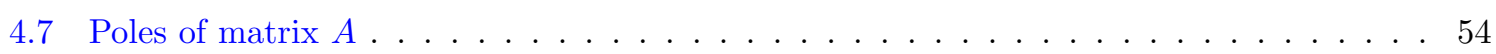

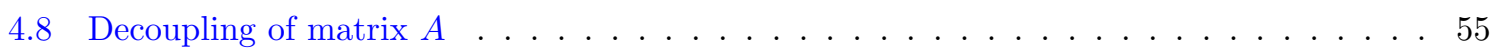

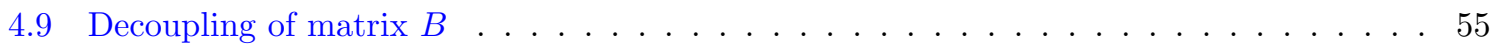

4.10 Spiral excitation with three different rudder pulses $\ldots \ldots \ldots \ldots \ldots$

5.1 Design Parameters . . . . . . . . . . . . . . . . . . . . . 71

5.2 Phugoid sensitivity to model parameters $\ldots \ldots \ldots \ldots \ldots \ldots \ldots$

5.3 Short period sensitivity to model parameters $\ldots \ldots \ldots \ldots \ldots \ldots$ 
5.4 Effect of $C_{L_{q}}$ on pull-up/push-over static characteristics $\ldots \ldots \ldots \ldots \ldots \ldots$

5.5 Roll performace sensitivity to model parameters $\ldots \ldots \ldots \ldots \ldots$

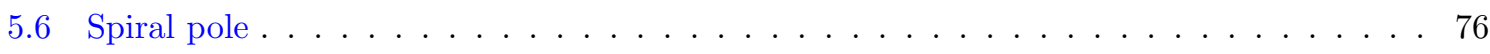

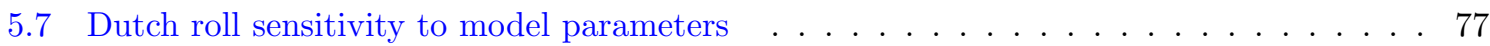

5.8 Lateral static characteristics sensitivity to $C_{n_{\delta_{a}}} \ldots \ldots \ldots \ldots \ldots \ldots$

5.9 Lateral static characteristics sensitivity to $C_{l_{\delta_{a}}} \ldots \ldots \ldots \ldots \ldots \ldots \ldots$

5.10 Lateral static characteristics sensitivity to $C_{l_{\delta_{r}}} \ldots \ldots \ldots \ldots \ldots$

5.11 Lateral static characteristics sensitivity to $C_{l_{\beta}} \ldots \ldots \ldots \ldots \ldots \ldots$

5.12 Effect of a $20 \%$ change in design parameters on static characteristics . . . . . . . . 88

5.13 Cessna 182. Reprinted from http://www.flugzeuinfo.net . . . . . . . . . . . . . . . 89

5.14 Beech 99. Reprinted from http://gallery.photo.net . . . . . . . . . . . . . . 89

6.1 Effect of design changes on elevator position stability . . . . . . . . . . . . . . . . . 94

6.2 Effect of design changes on rudder position directional stability $\ldots \ldots \ldots \ldots \ldots$

B.1 Sign convention for aerodynamic forces and angles . . . . . . . . . . . . 108

B.2 Control Surfaces . . . . . . . . . . . . . . . . . . . . . . . . . . . . 108

B.3 Geometric Parameters . . . . . . . . . . . . . . . . . . . . . . . . . . . 109

C.1 Geometric Parameters . . . . . . . . . . . . . . . . . . . . . . . . . . 118

C.2 Geometric Parameters . . . . . . . . . . . . . . . . . . . . . . . . 118

D.1 ANTEX model . . . . . . . . . . . . . . . . . . . . . . . . . . 121

D.2 Approximation of aircraft parts as standard geometric figures . . . . . . . . . . 123

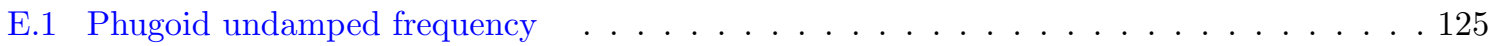

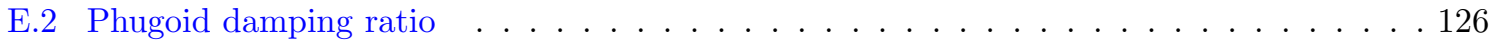

E.3 Short period undamped frequency . . . . . . . . . . . . . . . . . 126

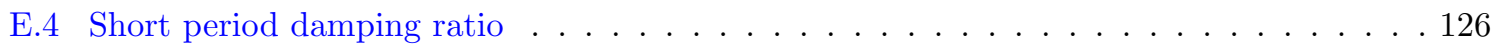

E.5 Roll pole . . . . . . . . . . . . . . . . . . . . . . . . . . . . . . . 127

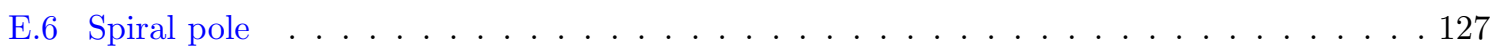

E.7 Dutch roll undamped frequency _ . . . . . . . . . . . . . . . . . . . . 127

E.8 Dutch roll damping ratio f . . . . . . . . . . . . . . . . . . . . 128 


\section{List of Tables}

2.1 Assumptions and validity of the dynamic model . . . . . . . . . . . . . . 11

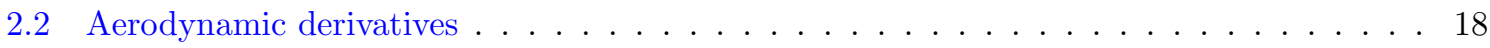

2.3 Steady-state flight conditions and characteristics . . . . . . . . . . . . . 22

3.1 Static flying qualities parameters $\ldots \ldots \ldots \ldots \ldots \ldots \ldots \ldots \ldots$

3.2 Dynamic flying qualities parameters $\ldots \ldots \ldots \ldots \ldots \ldots \ldots \ldots$

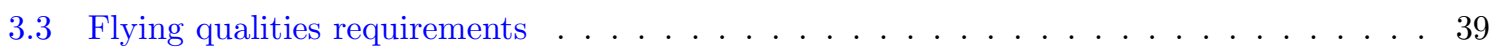

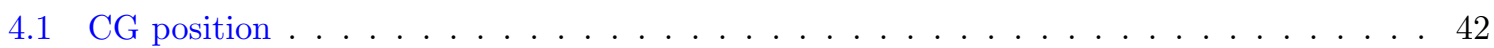

4.2 Longitudinal aerodynamic derivatives . . . . . . . . . . . . . . . . . 45

4.3 Lateral stability derivatives $\ldots \ldots \ldots \ldots \ldots \ldots \ldots \ldots$

4.4 Requirements for static stability $\ldots \ldots \ldots \ldots \ldots \ldots \ldots \ldots$

4.5 Maximum disturbances for linearisation validity . . . . . . . . . . . . . 53

4.6 Undamped frequency and damping ratio of the different modes . . . . . . . . . . . . 54

4.7 Characteristics of dynamic modes as predicted by the linearised decoupled model . . . . 56

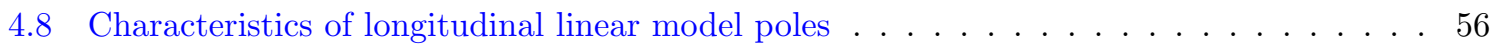

4.9 Characteristics of longitudinal submatrix poles, without engine dynamics . . . . . . 57

4.10 Characteristics of lateral linear model poles . . . . . . . . . . . . . . . 57

4.11 Time to double for spiral mode $\ldots \ldots \ldots \ldots \ldots \ldots$

4.12 Roll performance characteristics . . . . . . . . . . . . . . . . . . . . . . 59

4.13 Comparison between linearised model and nonlinear model results . . . . . . . . . . 65

4.14 Comparison between between linear decoupled model and linearised model results . . . 66

4.15 Flying qualities requirements for Class I, category B . . . . . . . . . . . 67

4.16 Comparison between results with and without spinning rotors effect $\ldots \ldots \ldots$. . . 68

5.1 Longitudinal flying qualities sensitivity to model parameters . . . . . . . . . . . 75

5.2 Lateral flying qualities sensitivity to model parameters . . . . . . . . . . . . . 78

5.3 Effect on mass properties . . . . . . . . . . . . . . . . . . . . . . 81

5.4 Longitudinal elements affected by design parameters $\ldots \ldots \ldots \ldots \ldots$

5.5 Lateral elements affected by design parameters . . . . . . . . . . . . . . . 83 
5.6 Effect of design changes on longitudinal derivatives $\ldots \ldots \ldots \ldots \ldots$

5.7 Effect of design changes on lateral derivatives . . . . . . . . . . . . . . . 85

5.8 Effects of a $\pm 20 \%$ change in design parameters on longitudinal flying qualites . . . . . 86

5.9 Effects of a $\pm 20 \%$ change in design parameters on lateral flying qualities $\ldots \ldots$. . . 87

5.10 Effects of a $20 \%$ change in design parameters on phugoid and spiral, using the linearised and nonlinear models . . . . . . . . . . . . . . . . . . . . . . 87

5.11 Effects of a $20 \%$ change in design parameters on drag $\ldots \ldots \ldots$. . . . . . . 87 


\section{List of Symbols and Abbreviations}

$\alpha \quad$ Angle of attack

$\bar{q} \quad$ dynamic pressure

$\beta \quad$ Sideslip angle

$\delta_{a} \quad$ Aileron deflection

$\delta_{e} \quad$ Elevator deflection

$\delta_{r} \quad$ Rudder deflection

$\delta_{t} \quad$ Throttle command

$\gamma \quad$ Flight path angle

$\lambda \quad$ Heading angle

$\omega_{n} \quad$ Undamped frequency

$\phi \quad$ Roll angle

$\psi \quad$ Yaw angle

$\rho \quad$ Air density

$\tau \quad$ Time constant

$\theta \quad$ Pitch angle

$\xi \quad$ Damping ratio

c Wing chord

$C_{D} \quad$ Drag coefficient

$C_{L} \quad$ Lift coefficient

$C_{l} \quad$ Rolling moment coefficient 
$C_{m} \quad$ Pitching moment coefficient

$C_{n} \quad$ Yawing moment coefficient

$C_{Y} \quad$ Side force coefficient

$d r \quad$ Dutch Roll

$p \quad$ Angular velocity about body $x$ axis

ph Phugoid

$q \quad$ Angular velocity about body $y$ axis

$r \quad$ Angular velocity about body $z$ axis

S Wing area

sp Short period

$T_{2} \quad$ Time to double amplitude

$u \quad$ Longitudinal speed

$V \quad$ Airspeed or just speed

$v \quad$ Lateral speed

$w \quad$ Vertical speed

CAD Computer-Aided Design

CFD Computational Fluid Dynamics

CG Center of gravity

DOF Degrees of freedom

FQ Flying Qualities

ISA International Standard Atmosphere

PoAF Portuguese Air Force

UAV Unmanned Air Vehicle 


\section{Chapter 1}

\section{Introduction}

\subsection{Objective}

Unmanned Air Vehicles (UAVs) have been gaining more and more adepts, both civil and military, due to their versatility, low cost (comparing to their larger counterparts) and the fact that they represent no risk for their operators. They have already proven to be adequate to different types of mission, and improvement of their performance and efficiency is an important research topic of the future. But this is a rich area, including subjects from system engineering, which is not necessarily concerned with the platform itself, to aerodynamics. Many different directions can be taken to contribute to UAV development.

Here, we focus on the platform, that is to say, on the aircraft design. The purpose is to parameterise how slight modifications on a baseline configuration can improve/deteriorate the response of the aircraft when accomplishing a certain task. And this is what flying qualities is about: the ease and effectiveness with which an aircraft responds to command inputs aiming at a certain mission.

\subsection{Motivation}

The idea for this project results from a common interest between the Portuguese Air Force (PoAF) and the Swedish Defense Research Agency (FOI).

The Portuguese Air Force Academy has, since 1999 (but gaining more relevance since last year), a large project in hands on UAV development, PITVANT, which has caught the attention of a few national and international entities, willing to cooperate. So far, some platforms have been developed and successfully tested in flight, both in RP (remotely piloted) and autonomous modes. These platforms have a common configuration/design that came out from studies and experience-based knowledge, regarding requisites like:

- Low cost 
- Fixed wing

- Simple geometry

- Optimised wing

- Endurance

- Ability to carry a certain amount of payload

The intention was to build a test platform, not a mission oriented UAV.

An important part of current research is related to the project of new vehicles for different missions, particularly surveillance-oriented.

It is mainly the mission that dictates the requirements an aircraft should meet. Because of its complex dynamics, the design that suits one task, or even one requirement, most probably will not suit another as well, and therefore trade-offs are constantly present in aircraft development. A possible and natural solution is to start from an existing configuration, with known dynamics, and to study how its response might be modified by the introduction of some changes. The result is a set of design directions to follow or avoid, regarding specific requirements.

FOI is currently working on a larger scheme, CESAR Project, where certain design parameters of a manned aircraft are to be varied in the design phase of the development. The objective is to reduce the costly testing and adaption which usually is necessary to meet the certification requirements. Parametric studies represent a valuable tool in this process.

Although the required flying qualities may differ between UAVs and manned aircraft, the procedure of analysing them over a parameterised set of designs is equally valuable in both cases.

\subsection{Problem Statement}

Consider the system of Figure 1.1.

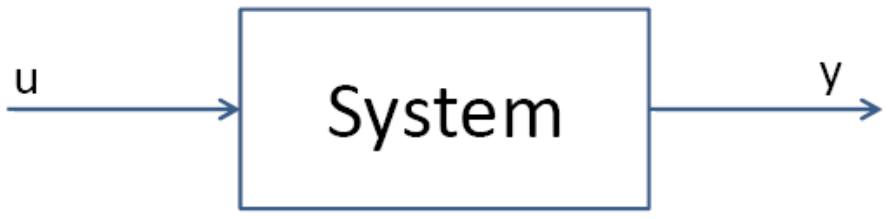

Figure 1.1: General open-loop system

By looking at the system output $y$, following a certain input $u$, it is possible to identify some of the characteristics that describe the dynamic behaviour of the system. 
Consider now that the system exists for a purpose/mission. Then, it is desirable that it reacts in a specific way to an input, whether it is deliberate or not. Based on the response, which can be analysed through $y$, but that only depends on its dynamics, the system can be said be adequate or not to the mission it was designed for.

The goal is to understand how some changes in the system can improve or deteriorate its dynamic behaviour.

The system we study is an aircraft, a PoAF UAV called ANTEX-M X03 (see Figure 1.2), and flying qualities are used as a measure of how well it performs a certain task.

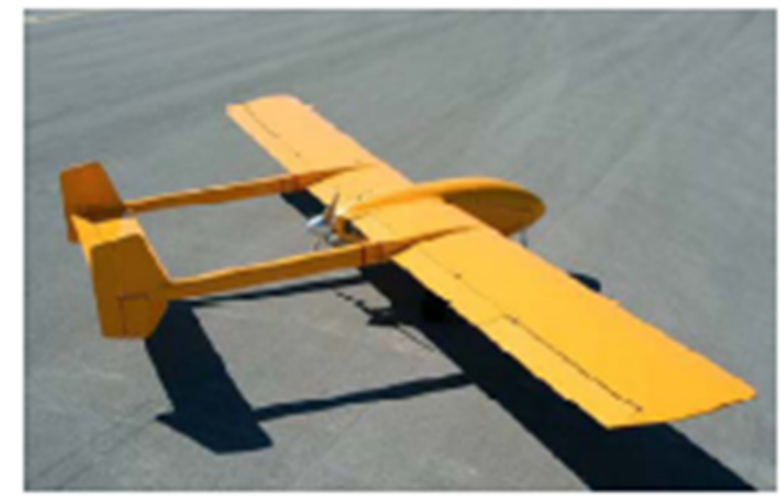

Figure 1.2: PoAF UAV ANTEX-M X03

By what was said above, two things are important when assessing flying qualities: the system itself and the mission. While mission defines the rating scale of flying qualities, the parameters that are actually rated depend mainly on the system.

This work is mainly focused on flying qualities parameters and not on requirements or ratings, because:

1. No specific requirements for UAVs exist so far

2. The same parameters are used irrespective of the mission

3. Changes to be made on the system reflect themselves on parameters, not requirements

Therefore, in order to meet the objective as stated in Section 1.1, it is important to get familiar with the system and its dependence on aircraft design. This study is based on a mathematical model, which can be very complex if a high level of detail is required. Usually, for the type of information we need, a 6-DOF nonlinear model with a simplified description of the aerodynamics is adequate. Its general form is independent of aircraft configuration and this property will soon reveal to be extremely useful for this project. Figure 1.3 shows the main components of this type of model.

The way design data is introduced in the model is mainly through the aerodynamic characteristics, which are usually cast into an aerodynamic model, and mass properties. 


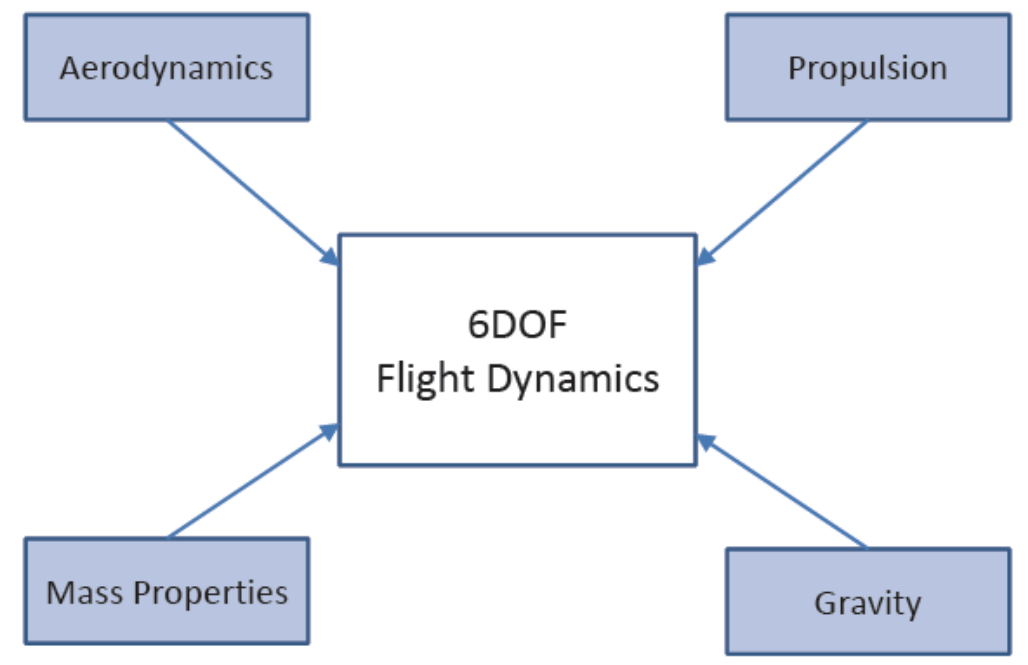

Figure 1.3: Main components of an aircraft 6-DOF dynamic model

Although analysis of how design changes affect flying qualities could be done in one step, this would lead to conclusions too dependent on initial design/configuration, which difficults results extrapolation to other aircraft types. Taking advantage of the fact that the dynamic model structure is independent of initial design, we split the analysis in two steps, according to Figure 1.4. Once the design and flying qualities parameters to study are chosen, the approach is as follows:

1. Analysis of flying qualities dependence on aerodynamic characteristics and mass properties - not dependent on configuration

2. Analysis of aerodynamic characteristics and mass properties dependence on design parameters dependent on configuration

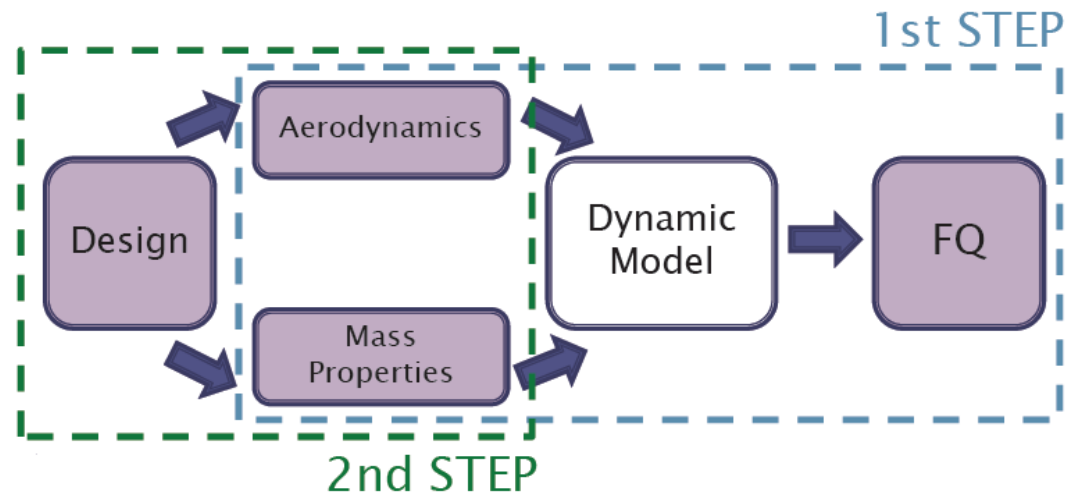

Figure 1.4: Strategy for parametric studies

Then, by putting together information got from both steps, it is possible to parameterise the influence of a set of design parameters on flying qualities, with the additional advantage that conclusions 
drawn from step one might be used for other aircraft configurations.

\subsection{Tools}

Development of any project requires the use of tools. Usually, they can be divided in two groups: references and specific software or equipment. The former is used for background support and to understand what has been done on the subject so far, to avoid duplication. The latter involves some instruments without which it would be difficult, or even not possible, to go forward with the project.

The following list shows the main tools we used for each phase of the project. The intention is to help the reader understand how the work was conducted and why it was conducted in a certain way, and also to show where to find further information on the different subjects.

\section{- Starting point:}

Some work has already been done on ANTEX-M X03 to determine its aerodynamic characteristics, mass properties and dynamic behaviour [9,6]. A summary of the available data on these subject can be found on Appendix A. There has been some difficulty measuring some parameters, which has led to unexpected results. Those parameters are identified in the same document and require validation

\section{- Dynamic Model:}

Section 1.3 called the reader's attention to the importance of understanding the dynamic model of an aircraft. References $([16,7,18])$ provide a detailed derivation of the equations of motion for an aircraft, as a rigid body, and are used as background for this subject.

\section{- Aerodynamic Model:}

The aerodynamic model of an aircraft can itself be as complex as the dynamic model. Although it has been determined by Félix and Saraiva [9] , there are some parameters missing and others to validate. Some theory on the usual approach to this topic can be found in references [21, 16]. We used two methods to determine and validate the model parameters: one based in computational simulation and other on existing empiric expressions, both widely used today, but in different stages of aircraft development. There is a number of CFD tools available in the market, but, as to the empiric expressions, DATCOM [10] is unanimously known to be the reference in the area. Therefore, aerodynamic model determination is based both on a CFD code developed at FOI (EDGE), and DATCOM.

\section{- Mass Properties:}

Mass properties include both aircraft center of gravity position and inertia matrix (related to mass distribution). Inertia matrix determination is where most difficulties were met: attempts to determine it experimentally were unsuccessful [9], probably because of the considerable dimension of the model; Coutinho ([6]) used estimated values based on existing aircraft/UAVs 
but uncertainty is quite significant. A different approach is followed here: by adaptation of an existing CAD 3D model of the aircraft, we use SolidWorks (3D CAD design software) to compute inertia matrix.

\section{- Flying Qualities:}

One of the most cited books on this topic is Hodgkinson, [12], but essentially from a piloted aircraft point of view. He identifies the parameters that most influence pilots' opinion and shows how to extract them from the dynamic model. Research on UAV flying qualities is very recent, but some $([3,5,13,11])$ have addressed the importance of creating specific requirements for unmanned aircraft. Therefore current assessment of UAV flying qualities is still based on manned requirements, which can be found on $([15,14])$.

- Model Analysis and Parametric Studies: The simulation environments used for this purpose are MATLAB and Simulink. Although not presented in this report, several MATLAB programs were written to perform the different analysis; others, for dynamic model implementation, were adapted from existing ones, developed by Coutinho[6] for his project.

\subsection{Report Outline}

The report is structured as follows:

Chapter 2. Dynamic Model: The general equations of motion for a rigid aircraft are derived, under certain assumptions. From all the forces acting on the body, special attention is given to the aerodynamic ones. The result is a 6-DOF nonlinear model, which is then linearised around a specific flight/trim condition, because a linear model can provide relevant information and is not as complex to deal with. The validity of the linear model, however, is confined to a small envelope around the trim condition. Although the general form for an aircraft linear model is available in literature, algorithms to trim the model, linearise it around the trim point and define linearisation validity limits are also presented.

Chapter 3. Flying Qualities: A brief overview on this topic is presented: definition, origin, relevance and typical requirements for piloted aircraft, which depend on specific parameters describing the aircraft dynamic behaviour. Then, some of these parameters are selected for analysis of UAV flying qualities, according to their importance and applicability to unmanned aircraft. Finally, it is explained how to extract them from the dynamic model.

Chapter 4. ANTEX-M X03 Flying Qualities: The first part of this chapter is dedicated to determining/validating data for dynamic model derivation. Mass properties are determined computationally and aerodynamic characteristics through both CFD and empiric expressions. Results are 
then compared with existing ones, and, based on the conclusions drawn, the ones that seem more reasonable are used to build the dynamic model. Afterwards, flying qualities parameters from Chapter 3 are determined using the nonlinear and linearised models, as well as the general linear model. The objective is to compare the results and check if the last one, which is simpler to obtain and gives more physical insight to aircraft behaviour, can be used for further analysis.

Chapter 5. Parametric Studies: After choosing some design parameters, we study their influence on flying qualities. The analysis is divided in two steps, as explained on Section 1.3. Then, the influence of chosen design variables on flying qualities is parameterised. The model used for the analysis is the general linear model.

Chapter 6. Improving ANTEX-M X03 Design: Based on results from previous chapter, a design option is suggested in order to improve ANTEX-M X03 flying qualities, regarding a specific mission, with specific requirements, and considering a set of restrictions. Then, the consequences for the different flying qualities parameters are analysed.

Chapter 7. Conclusions and Future Work: The last chapter is a summary of the work done and conclusions drawn throughout the project. And because this is such a vast area, to which this project gives its contribution, some suggestions for future work are presented. 


\section{Chapter 2}

\section{Dynamic Model of an Aircraft}

Flight testing is still the most accurate way of studying the dynamics of motion of an aircraft, but not the most efficient: not only can it become extremely expensive (depending on the aircraft) but also quite limited if the purpose is to study the effects of introducing some modifications. Computational simulations appear, then, as an important tool: cheaper and at the same time more versatile.

To run these simulations, though, a mathematical model of the aircraft dynamics is required. And a model, as good as it can get, will always be an approximation. Depending on the detail or precision needed (or wanted), the dynamic model of an aircraft can be very complex. Nevertheless, according to the type of analysis (features to study) to perform, it is possible to introduce some simplifications and still get a rather good approximation.

\subsection{Dynamic Model Derivation}

This section presents a brief description of how this model can be derived. During the process, some assumptions are made which greatly simplify it. These are frequently found in flight mechanics/dynamics books and studies similar to the ones carried out in this project, which lead to simpler models that have proven to be very useful in practice, see e.g. Etkin [7].

The first assumption is that the aircraft moves as a rigid body, in six degrees of freedom. As a consequence, and according to classic mechanics, on the basis of its dynamics is Newton's Second Law, which states:

$$
\begin{aligned}
M & =\dot{h} \\
F & =\dot{p}
\end{aligned}
$$


where $M$ is the sum of external moments acting on the body, relative to its centre of mass/gravity $(\mathrm{CG})^{1}, F$ the sum of external forces, $h$ and $p$ the angular momentum and momentum of the body, respectively, given by:

$$
\begin{gathered}
h=I \omega \\
F=m V
\end{gathered}
$$

where $I=\left[\begin{array}{rrr}I_{x x} & -I_{x y} & -I_{x z} \\ -I_{x y} & I_{y y} & -I_{y z} \\ -I_{x z} & -I_{y z} & I_{z z}\end{array}\right]$ is the inertia matrix of the body, $\omega$ its angular velocity, $m$ its mass and $V$ linear velocity, or just velocity.

This law is only valid on an inertial reference frame (unaccelerated). However, it is more convenient to use a frame moving with the body (aircraft) where the origin is coincident with its CG. The advantages of using such frame are:

- Physical insight

- Constant inertia tensor, if changes in mass are neglected

- Translational and angular motions independent

If equations (2.1) are to be used in a body-fixed frame, an adjustment is required.

Lemma 1 Let $A$ an inertial and frame of reference and $B$ another frame fixed in a body moving relative to $A$. If $z$ is a vector defined on frame $A$, then

$$
\dot{z}_{A}=\dot{z}_{B}+\omega_{B / A} \times z
$$

where the subscript $A$ indicates derivation on frame $A, B$ derivation on frame $B$, and $\omega_{B / A}$ is the angular velocity of $B$ relative to $A$.

Proof of Lemma 1 can be found in [18].

At this time, two reference frames will be used: an inertial one, so Newton's Second Law is valid, and another fixed in the aircraft, for convenience. Before moving on with the model derivation, some additional assumptions are made:

- The Earth is flat and stationary

- The mass of the aircraft is constant

- Longitudinal symmetry 


\begin{tabular}{|c|c|c|}
\hline Assumption & Validity & Consequences \\
\hline Flat Earth & \multirow[t]{2}{*}{$\begin{array}{l}\text { Low Speed, low altitude, } \\
\text { small range }\end{array}$} & $\begin{array}{l}\text { Effects due to Earth shape ne- } \\
\text { glected }\end{array}$ \\
\hline Stationary Earth & & Inertial Frame \\
\hline Constant Mass & $\begin{array}{l}\text { Simulation time is small } \\
\text { enough to neglect mass varia- } \\
\text { tion; no payload drop }\end{array}$ & $\begin{array}{l}\text { CG position and Inertia Tensor } \\
\text { are Constant }\end{array}$ \\
\hline Longitudinal Symmetry & Typical of conventional aircraft & $I_{x y}$ and $I_{y z}$ are null \\
\hline
\end{tabular}

Table 2.1: Assumptions and validity of the dynamic model

Table 2.1 shows why the above statements can be considered valid for the UAV under study, and the resultant consequences that make the model simpler. For more information on this subject, including associated limitations of the model, the interested reader is referred to [18].

If the Earth is considered flat and stationary, we can use it as an inertial frame. This frame is defined by having its origin on the surface, $x$ pointing North, $y$ pointing East and $z$ pointing Down, and that is why it is also know as NED. For the body-fixed frame, the convention used is presented in Figure 2.1.

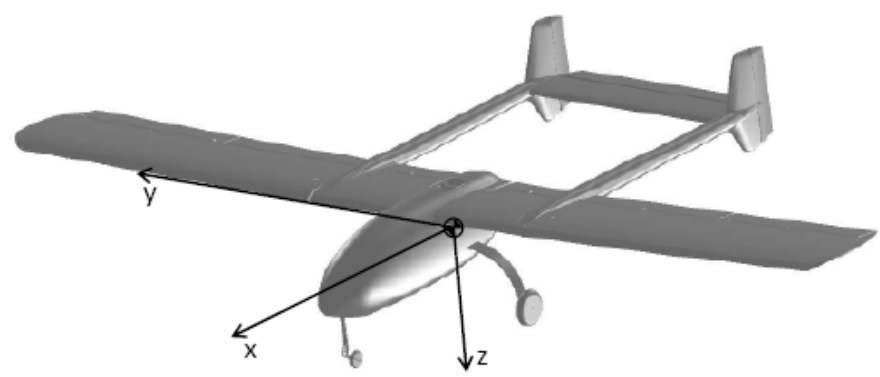

Figure 2.1: Boxy axes

Considering previous assumptions and using Lemma 1, equations (2.1) become:

$$
\begin{aligned}
M_{b} & =I_{b} \dot{\omega}+\omega \times\left(I_{b} \omega\right) \\
F_{b} & =m\left(\dot{V}_{b}+\omega \times V_{b}\right)
\end{aligned}
$$

Equations (2.4) are the dynamic equations and they completely describe the aircraft motion, on its reference frame, identified by subscript $b$. To describe its motion relative to the Earth, though, kinematic equations are also required.

\footnotetext{
${ }^{1}$ Under the assumption of uniform gravity, center of mass and center of gravity are coincident.
} 
The first step is to orient the aircraft relative to the Earth-fixed frame.

Lemma 2 Let $A$ and $B$ be two different coordinate systems. By three successive rotations of $A$ about its axes, it is always possible to orient it parallel to B. The rotation angles are known as Euler angles and define the orientation of $B$ with respect to $A$.

Proof of Lemma 2 can be found in [18].

In the aeronautical field these rotations are performed as follows:

1. Right-handed rotation about the z-axis (positive psi)

2. Right-handed rotation about the new y-axis (positive $\theta$ )

3. Right-handed rotation about the new x-axis (positive $\phi$ )

The angles $\psi, \theta, \phi$ are known as yaw, pitch and roll angles, respectively. Usually the pitch angle variation is restricted to $\pm \frac{\pi}{2}$, so that a given orientation is described by a single combination of the Euler angles.

The aircraft angular velocity relative to the Earth is given by:

$$
\omega=C_{b / e}\left\{\begin{array}{c}
\dot{\phi} \\
\dot{\theta} \\
\dot{\phi}
\end{array}\right\}
$$

where

$$
C_{b / e}=\left[\begin{array}{ccc}
1 & 0 & -s \theta \\
0 & c \phi & s \phi c \theta \\
0 & -s \phi & c \phi c \theta
\end{array}\right]
$$

The inverse relation is

$$
\left\{\begin{array}{c}
\dot{\phi} \\
\dot{\theta} \\
\dot{\phi}
\end{array}\right\}=C_{b / e}^{-1} \omega
$$

where

$$
C_{b / e}^{-1}=C_{e / b}=\left[\begin{array}{ccc}
1 & t \theta s \phi & t \theta c \phi \\
0 & c \phi & -s \phi \\
0 & -s \phi / c \theta & c \phi / c \theta
\end{array}\right]
$$

On matrices (2.6) and (2.8), $c, s$ and $t$ represent cosine, sine and tangent, respectively.

From equation 2.7 it can be seen that, when $\theta$ reaches $\frac{\pi}{2}$, a singularity arises which can lead to simulation problems. To avoid this, the quaternion method can be used. Although its formulation 
is numerically preferable, the simulated flight envelope on this project is not very wide, at least in terms of pitch angle, and so Euler simulation is enough. Moreover, it gives more physical insight to the aircraft attitude.

More information about the quaternion method can be found in e.g. [18].

By knowledge of aircraft orientation, its flight path can be tracked through another set of three equations:

$$
\left\{\begin{array}{l}
\dot{x}_{e} \\
\dot{y}_{e} \\
\dot{z}_{e}
\end{array}\right\}=\left[\begin{array}{ccc}
c \theta c \psi & s \phi s \theta c \psi-c \phi s \psi & c \phi s \theta c \psi+s \phi s \psi \\
c \theta s \psi & s \phi s \theta s \psi+c \phi c \psi & c \phi s \theta s \psi-s \phi c \psi \\
-s \theta & s \phi c \theta & c \phi c \theta
\end{array}\right] V_{b}
$$

The equations derived previously did not take into consideration the possible existence of wind, that is to say, a nonzero wind velocity vector, which would require an update to the equation, through the velocity vector (see e.g. Etkin [7]). But for the purpose of this work, no wind will be considered.

\section{Effect of Spinning Rotors}

Usually the propulsion systems that equip aircraft, either propeller or turbine engines, are composed by spinning rotors which create gyroscopic moments. In the case of counter-rotating rotors, this effect can be neglected. However, this is not the case of the our UAV, which has only one propeller. To account for this, a single term $M_{b}^{\prime}$ must be added to the moment equation (2.4a), due to the angular moment $h^{\prime}$ of the spinning rotors.

Lemma 3 Let $I_{r}$ be the moment of inertia of the rotor about its axis and $\omega$ its spinning velocity.

$$
h^{\prime}=I_{r} \omega
$$

Expanding the new term according to Lemma 1, and assuming a constant $h^{\prime}$

$$
M_{b}^{\prime}=\left\{\begin{array}{c}
q h_{z}^{\prime}-r h_{y}^{\prime} \\
r h_{x}^{\prime}-p h_{z}^{\prime} \\
p h_{y}^{\prime}-q h_{x}^{\prime}
\end{array}\right\}
$$

For a propeller engine with its spinning axis aligned with the longitudinal axis of the aircraft

$$
h^{\prime}=\left[\begin{array}{lll}
h_{x}^{\prime} & 0 & 0
\end{array}\right]^{T}
$$

These effects will not be considered, but the consequences of doing so are analysed on Chapter 4 .

\subsubsection{The Equations Collected}

For more simplicity and insight, the equations are now cast in their scalar form. From now on, only the body frame is considered and we will drop subscript $b$. 
The following notation is used: $F=\left\{\begin{array}{c}X \\ Y \\ Z\end{array}\right\}, M=\left\{\begin{array}{c}L \\ M \\ N\end{array}\right\}, V=\left\{\begin{array}{c}u \\ v \\ w\end{array}\right\}$ and $\omega=\left\{\begin{array}{l}p \\ q \\ r\end{array}\right\}$.

Dynamic Equations

$$
\begin{aligned}
X & =m(\dot{u}+q w-r v) \\
Y & =m(\dot{v}+r u-p w) \\
Z & =m(\dot{w}+p v-q u) \\
L & =I_{x} \dot{p}-I_{z x} \dot{r}+q r\left(I_{z}-I_{y}-I_{x z}\right) p q \\
M & =I_{y} \dot{q}+r p\left(I_{x}-I_{z}\right)+I_{x z}\left(p^{2}-r^{2}\right) \\
N & =I_{z} \dot{r}-I_{x z} \dot{p}+p q\left(I_{y}-I_{x}\right)+I_{x z} q r
\end{aligned}
$$

Kinematic Equations

$$
\begin{aligned}
\dot{\phi} & =p+(q \sin \phi+r \cos \phi) \tan \theta \\
\dot{\theta} & =q \cos \phi-r \sin \phi \\
\dot{\psi} & =(q \sin \phi+r \cos \phi) \sec \theta
\end{aligned}
$$

\section{Navigation Equations}

$$
\begin{aligned}
\dot{x}_{e}= & u \cos \theta \cos \phi+v(\sin \phi \sin \theta \cos \psi-\cos \phi \sin \phi) \\
& +w(\cos \phi \sin \theta \cos \psi+\sin \phi \sin \psi) \\
\dot{y}_{e}= & u \cos \theta \sin \psi+v(\sin \phi \sin \theta \sin \psi+\cos \phi \cos \psi) \\
& +w(\cos \phi \sin \theta \sin \psi-\sin \phi \cos \psi) \\
\dot{z}_{e}= & -u \sin \theta+v \sin \phi \cos \theta+w \cos \phi \cos \theta
\end{aligned}
$$

\subsubsection{Forces and Moments}

To finish model derivation, the forces and moments appearing in equations (2.13) need to be modeled themselves. They result from the sum of gravitational, aerodynamic and thrust forces and moments.

Aerodynamic forces and moments are presented in a separate section, due to their complexity.

\subsubsection{Gravitational Force}

The gravitational force vector, usually known as weight, is given by the following relation: 


$$
W=m g\left\{\begin{array}{c}
\sin \theta \\
\cos \theta \sin \phi \\
\cos \theta \cos \phi
\end{array}\right\}
$$

For proof, see Etkin [7, pp. 101].

Because it is applied on body $\mathrm{CG}$, there are no resulting moments.

\subsubsection{Thrust Force and Moment}

They depend on the dynamics of the propulsion system. On reference [6] the reader can find a model for a propeller engine similar to the one that equips the UAV. The author does not find it essential to be present it here, though it is important to transmit the idea that the thrust force and moment transmitted by the engine depend on the outside pressure and throttle command. For a single propeller engine with its spinning axis aligned with the longitudinal axis of the aircraft, the thrust force is

$$
F_{T}=\left[\begin{array}{lll}
T & 0 & 0
\end{array}\right]^{T}
$$

Propeller rotation causes a reaction rolling moment on the aircraft, also known as propeller torque and so:

$$
M_{T}=\left[\begin{array}{lll}
-Q & 0 & 0
\end{array}\right]^{T}
$$

where $Q$ is the moment transmitted by the engine.

\subsubsection{Aerodynamic Model}

Finding a suitable model for aerodynamic forces and moments is probably one the most difficult steps for studying flight dynamics, because they are unknown functions of Reynolds and Mach numbers, aircraft geometry and attitude relative to the flow, and depend on the history of these parameters during the flight.

A rigorous model can then be very complex and difficult to handle mathematically, and there will always be a tradeoff between accuracy and complexity. Fortunately, it is possible to come up with approximations with an acceptable level of accuracy, which not only make the model simpler but also measurements, though they are only valid for a limited flight envelope. The minimum accuracy required depends on the kind of studies to perform.

Usually, aerodynamic forces and moments (see Appendix B) are studied in their dimensionless form, the so called aerodynamic coefficients, which are obtained dividing forces by the product of dynamic pressure $\bar{q}$ and wing surface area $S$, and moments by the product of dynamic pressure, wing surface 
area and a characteristic lenght: wing chord $c$ for $M$, and wing span $b$ for $L$ and $N$. Dynamic pressure is given by:

$$
\bar{q}=\frac{1}{2} \rho V^{2}
$$

where $\rho$ is the air density and $V$ is the aircraft speed.

The two most commonly used methods for modelling these coefficients are presented below:

\section{- Lookup-Tables:}

They gather discrete values of the coefficients for numerous flight conditions. Data for an arbitrary flight condition is obtained by interpolation. By reducing the interval by which the different parameters (that define those flight conditions) vary, it is possible to get a very rigorous model. Nevertheless, the complex dependence on a lot of variables would lead to multidimensional tables, whose interpolation would be difficult, and to the need of a large number of tests. Besides, this method gives poor physical insight because it is difficult to identify the contribution of the different aircraft components.

\section{- Component Buildup:}

Coefficients are obtained by summing the contribution from different components, promoting a better understanding of the physics behind aircraft aerodynamics. Each contribution can be modelled through functions or lookup-tables. This approach has less dimensions and can be linearised. An additional advantage is that it is straightforward to add/remove the effect of one component, in the case of an aircraft modification.

\subsubsection{Aerodynamic Model Derivation}

The main objective of this project is to perform parametric studies. Having in mind the advantages and disadvantages of both the lookup-table and component buildup, the latter seems more adequate. This was also the approach followed by Félix [9] and Coutinho [6], whose results can be used, although some need validation. The component buildup resulted from a linearisation around a reference flight condition, that is to say a Taylor series expansion, keeping only the first order terms. We use the same method will be used.

It should be noted that linearisation is only valid for small disturbances from the reference state. In spite of this limitation, this approximation is widely used for studies where the simulation envelope is not too wide (not involving large changes in state variables - equation (2.21), like the one presented in Appendix A.

As a result of linearisation, each coefficient takes the following form:

$$
C\left(x_{0}+\delta x, u_{0}+\delta u\right)=C\left(x_{0}, u_{0}\right)+\left.\frac{\partial C}{\partial x}\right|_{x_{0}} \delta x+\left.\frac{\partial C}{\partial u}\right|_{u_{0}} \delta u
$$


where $x$ is the state vector given by

$$
x=\{u v w p q r \dot{u} \dot{v} \dot{w}\}^{T}
$$

$u$ the vector of controls (see Appendix B, Figure B.2):

$$
u=\{\delta e \delta a \delta r\}^{T}
$$

and $x_{0}$ and $u_{0}$ are the state and control vectors defining the reference flight condition, respectively.

The terms $\frac{\partial C}{\partial x}$ are usually known as stability derivatives and $\frac{\partial C}{\partial u}$ as control derivatives.

Usually, the aerodynamic angles $\alpha$ (angle of attack) and $\beta$ (sideslip angle) are used instead of $v$ and $w$, respectively (see Appendix B, Figure B.1 for aerodynamic angles definition). They can be determined through the following expressions:

$$
\begin{aligned}
& \alpha=\arctan \frac{w}{u} \\
& \beta=\arcsin \frac{v}{V}
\end{aligned}
$$

Although this is a simple model, including all first order terms means determining 78 derivatives. However, because of the mirror symmetry of our UAV (as in typical aircraft), important simplifications appear: using the Principle of Material Indifference, Zipfel[21] proved that, in the presence of mirror symmetry, some of the derivatives vanish, in a way that coupling between longitudinal and lateral modes ceases to exist (this is valid for first order terms only). This means that, in a first order approach, longitudinal (lateral) forces/moments are only affected by perturbations of the longitudinal (lateral) parameters.

As far as the lateral mode is concerned, this is an intuitive conclusion. Consider a symmetrical flight, where lateral forces and moments are null. If symmetry exists with respect to the longitudinal plane, then perturbations on that plane will produce identical and opposite reactions on both sides which sum up to zero, not affecting the initial value of the lateral forces and moments. The same does not happen with the longitudinal mode, although it can be assumed it does, with acceptable level of accuracy, at least for small disturbances.

Decoupling is a big simplification, but, like already mentioned, this is only valid for the first order terms. Coupling is actually present in higher order. On the other hand, the existence of a propeller, as is the case of ANTEX-M X03, breaks the symmetry, thus introducing coupling. This effect will not be considered.

Aerodynamic model derivation will then be based on the following assumptions:

- Linearisation

- Decoupling

\section{Stability Derivatives}




\begin{tabular}{|l|l|l|l|}
\hline \multicolumn{2}{|c|}{ Longitudinal Derivatives } & \multicolumn{2}{c|}{ Lateral Derivatives } \\
\hline \hline & \multicolumn{1}{|c|}{${ }^{2}$} & \\
Lift & $C_{L_{\alpha}}, C_{L_{q}}, C_{L_{\dot{\alpha}}}, C_{L_{\delta_{e}}}$ & Side Force & $C_{Y_{\beta}}, C_{Y_{p}}, C_{Y_{r}}, C_{Y_{\delta_{a}}}, C_{Y_{\delta_{r}}}$ \\
Drag & $C_{D_{\alpha}}, C_{D_{\delta_{e}}}$ & Rolling Mom. & $C_{l_{\beta}}, C_{l_{p}}, C_{l_{r}}, C_{l_{\delta_{a}}}, C_{l_{\delta_{r}}}$ \\
Pitching Mom. & $C_{m_{\alpha}}, C_{m_{q}}, C_{m_{\dot{\alpha}}}, C_{m_{\delta_{e}}}$ & Yawing Mom. & $C_{n_{\beta}}, C_{n_{p}}, C_{n_{r}}, C_{n_{\delta_{a}}}, C_{n_{\delta_{r}}}$ \\
\hline
\end{tabular}

Table 2.2: Aerodynamic derivatives

Taking into consideration the relative importance between the derivatives, additional simplification of the model is possible if the less significative are neglected. Usually, $\dot{u}$ and $\dot{\beta}$ derivatives, $C_{D_{\alpha}}$ and $C_{D_{q}}$ are not considered. Moreover, for low subsonic regime, which is the case of ANTEX-M X03 (see Appendix A), $u$ derivatives can also be neglected.

Consequently, the stability derivatives reduce to the ones on Table 2.2.

Derivatives relative to $p, q$ and $r$ have units of $s / \mathrm{rad}$. In order to nondimensionalise them, it is necessary to divide them by the following terms:

- $q$ :

$$
\frac{2 V}{c}
$$

- $p$ and $r$ :

$$
\frac{2 V}{b}
$$

Derivatives from Table 2.2 depend on the aerodynamic angles ( $\alpha$ and $\beta$ ), Mach number and Reynolds number. For the envelope of interest (see Appendix A), this variation is not significative and will not be accounted for.

\subsubsection{Data Gathering}

The aerodynamic characteristics, which, in this case, take the form of several derivatives, can be determined through the following methods:

- Flight Tests

- Wind Tunnel

- $\mathrm{CFD}$

- Empiric Expressions

The first one is the most expensive, but the most accurate. The second may imply the construction of a reduced-scale model. Simulations through CFD software usually take less time than the previous 
ones and can be quite precise. The last one is the less accurate, but provides quick and good estimation of different components contribution. The choice of the method depends on the available means, required accuracy and time to dispend.

\subsection{Atmospheric Model}

Air density and pressure $p_{a t m}$ decrease with altitude ant this affects both engine performance and dynamic pressure. Because UAVs usually fly at low altitudes, these effects will not be considered. Instead, it will be assumed that density and pressure are constant and equal to sea level values for a standard atmosphere, also known as ISA:

$$
\begin{aligned}
\rho & =1.225 \mathrm{~kg} / \mathrm{m}^{3} \\
p_{a t m} & =1013.2 \mathrm{hPa}
\end{aligned}
$$

\subsection{Trim}

\subsubsection{Overview}

The starting point for stability and performance analysis of an aircraft is a certain flight/trim condition. There is particular interest in steady-state/equilibrium flight conditions, where all motion variables, linear and angular velocities, are constant or zero.

The dynamic model of an aircraft is defined by a set of equations that can be written like

$$
\dot{x}=f(x, u)
$$

where $x=\{u v w p q \operatorname{pr} \theta \psi\}^{T}$ is the state vector and $u$ the control vector, as in equation 2.22.

The equilibrium points, as defined above, can then be found by making:

$$
\dot{x}=0
$$

Under the assumptions of flat Earth and negligible density variation with altitude, there is no coupling between navigation and dynamic equations, and the same happens with variable $\psi$. Therefore, no restrictions (like the one from equation (2.25)) need to be imposed on variables $x_{e}, y_{e}, z_{e}$ and $\psi$.

When trying to solve equation (2.25), not all the states are unknown, otherwise multiple solutions would come out, and not all would be feasible or interesting. So, to determine a steady-state, some states of the state vector are imposed, like airspeed, altitude and flight path angle, which define a specific trajectory of interest. The same can be done with some entries of the control vector. As to the unknown states and controls, it is possible to divide them in two groups:

- Derived parameters: consequence of trajectory restrictions 
- Control parameters: independent variables that are solution to the system of equations 2.25

By introducing some constraint equations, it is possible to reduce the set of independent variables, simplifying the system to be solved. An example of constraint equation is the relation between Euler and aerodynamic angles, which will be looked into right away. Let $V$ be the velocity vector in the wind frame ${ }^{2}$ and $V\left\{\begin{array}{c}* \\ \cos \gamma \cos \lambda \\ -\sin \gamma\end{array}\right\}$ the velocity in the Earth-fixed frame ${ }^{3}$. The two are related by:

$$
V\left\{\begin{array}{c}
* \\
\cos \gamma \cos \lambda \\
-\sin \gamma
\end{array}\right\}=C_{e / b} C_{b / w}\left\{\begin{array}{l}
V \\
0 \\
0
\end{array}\right\}
$$

where $\lambda$ is the heading angle, $\gamma$ the flight path angle, $C_{e / b}$ is as defined in equation (2.8) and

$$
C_{b / w}=\left[\begin{array}{ccc}
\cos \alpha \cos \beta & -\cos \alpha \sin \beta & -\sin \alpha \\
\sin \beta & \cos \beta & 0 \\
\sin \alpha \cos \beta & -\sin \alpha \sin \beta & \cos \alpha
\end{array}\right]
$$

Solving equation 2.26 leads to:

$$
\begin{aligned}
\sin \gamma & =\cos \alpha \cos \beta \sin \theta-(\sin \phi \sin \beta+\cos \phi \sin \alpha \cos \beta) \cos \theta \\
\cos \gamma \cos \lambda & =\cos \theta \cos \psi \cos \alpha \cos \beta \\
& +(\sin \phi \sin \theta \cos \psi+\cos \phi \sin \psi) \sin \beta \\
& +(\cos \phi \sin \theta \cos \psi+\sin \phi \sin \psi) \sin \alpha \cos \beta
\end{aligned}
$$

\subsubsection{Trim Conditions}

It was mentioned previously the existence of some trajectories of interest. In fact, for stability and performance analysis, there are specific steady-state flight conditions that cannot be disregarded, as they represent the basic flight modes of an aircraft. Apart from take-off and landing, which are beyond the scope of this work, the flight conditions of relevance are:

- Straight Level Flight: flight along rectilinear trajectory, with or without constant altitude. For a longitudinal symmetric aircraft, where flow pass it is also symmetric, it is possible to find a purely longitudinal solution, meaning lateral parameters equal to zero. Airspeed, altitude and flight path angle are set a priori.

- Steady-Sideslip: airspeed, altitude and sideslip are set a priori. We consider the case where yaw angle $\psi$ is held constant. This trajectory is also known as steady heading sideslip.

\footnotetext{
${ }^{2}$ The wind frame has its $x$ axis aligned with the velocity vector; $y$ and $z$ axes directions are not relevant for this work.

${ }^{3}$ The $*$ means that the corresponding component is not relevant.
} 
- Pull-up/push-over: though it is not a steady state, because $\dot{\theta}$ isn't zero, this is a maneuver worth studying. It is defined by a constant $q$. As in the case of straight level flight, a purely longitudinal solution is also possible. Airspeed, altitude, flight path angle and load factor $n^{4}$ are set a priori. It is the load factor that determines whether it is a pull-up or push-over: $n>1$ for pull-up and $n<1$ for push-over.

- Coordinated Turn:the aircraft follows a circular trajectory, with or without constant altitude, and null lateral forces. The case of zero sideslip is considered. Airspeed, altitude, flight path angle and load factor are set a priori.

In Table 2.3 there is a mathematical formulation for each steady-state flight condition, which includes initial conditions, derived and control parameters, and constraint equations.

\subsubsection{Trim Algorithm}

Because the aircraft model in nonlinear, finding a trim condition usually implies the use of numerical methods. The algorithm chosen to find the solution is based on the minimization of a nonnegative scalar cost function;it is an optimisation problem. A good candidate function (see e.g. [18]) is one that results from a linear combination of the square of state derivatives:

$$
C=\dot{x}^{T} G \dot{x}
$$

where $G$ is a weighting matrix to compensate for the possible order of magnitude difference between the various parameters. This is a direct search method because it uses optimisation techniques without derivatives. The order of magnitude of the cost function reflects the proximity between the numerical and real solution(it approaches zero as the numerical solution approaches the real one). MATLAB has as internal function for minima finding, fminsearch, based on Nelder and Mead Simplex Algorithm ${ }^{5}$. This function requires an initial guess. To find the trim condition, we developed a Matlab program which uses fminsearch. Once the program returns a solution, it is important to verify whether it is feasible, that is to say if it is inside the flight envelope and control surfaces limits.

We will use $C<0.001$ as convergence criterion.

\subsection{Linearisation}

\subsubsection{Overview}

We have presented a 6-DOF nonlinear model to describe flight dynamics of an aircraft. When running simulations for evaluation of aircraft performance, the nonlinear model is generally used [18,

\footnotetext{
${ }^{4}$ Load factor is the ratio of lift to weight

${ }^{5}$ More information on this algorithm can be found on MATLAB help menu.
} 


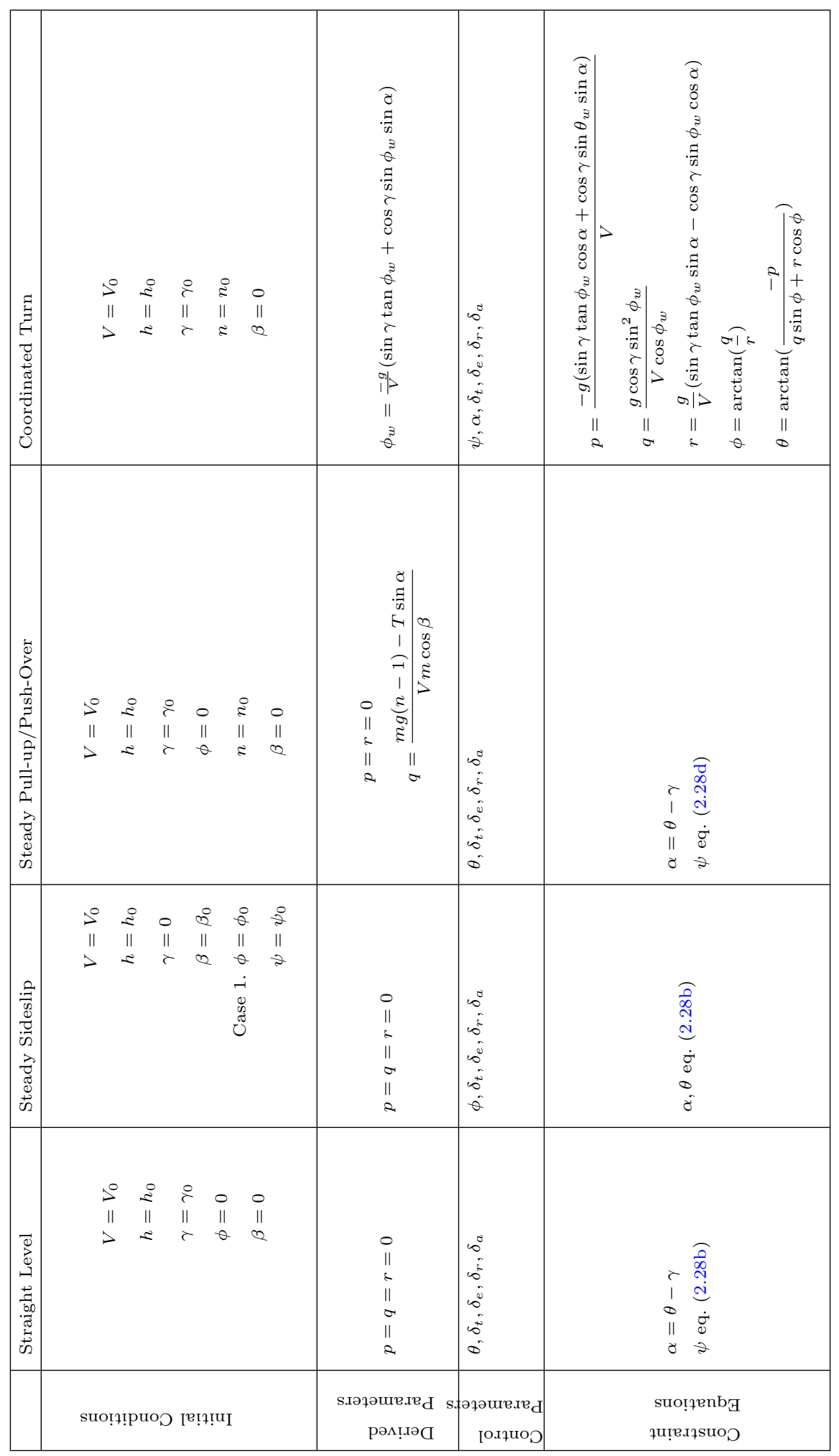

Table 2.3: Steady-state flight conditions and characteristics 
pp. 116]. Conversely, control system design techniques, which is outside the scope of this work, and stability studies are based on linear theory.

Although the nonlinear model is more accurate, it is usually difficult to work with analytically and there is a need to simplify it somehow, but still controlling the consequent inaccuracies. This can be done using small disturbance equations, which result in a linear system, much easier to deal with. However, this model is only valid for small disturbances around a trim condition/steady-state flight condition. Nevertheless, these conditions make up most of the flight time of an aircraft [7] and there is no interest in having large perturbations. Even fighter and attack airplanes operate mostly under conditions where small perturbations are desired.

As to the inaccuracies, they are reduced if simulations do not exceed small disturbances limits. In fact, small disturbances equations have proven, in many cases, to acceptably represent the dynamic response of an aircraft [17]. Additionally, a linear approach is very useful, as it provides more insight to the essential physical phenomena, allowing the identification of the relevant parameters.

In this work, the mathematical model of the UAV will be used primarily to perform two different analysis: stability and open-loop response. For a stability study, it is enough to use a linear model. On the contrary, when evaluating open-loop response, where significative disturbances are likely to occur, neglecting nonlinear effect might lead to significant errors. We will address to this problem later on Chapter 4 .

\subsubsection{Small Disturbances Equations}

Small disturbance equations are linear equations that follow from rewriting all variables of the original model as a sum of a reference value and a perturbation around it,

$$
x=x_{0}+\Delta x
$$

and neglecting second order perturbations.

Applying the above to dynamic and kinematic equations ${ }^{6}((2.13)$ and $(2.13))$, and assuming

$$
\begin{aligned}
& \cos \varepsilon \simeq 1 \\
& \sin \varepsilon \simeq \varepsilon
\end{aligned}
$$

where $\varepsilon$ is any small angle, the result, already simplifyed, is:

\footnotetext{
${ }^{6}$ Navigation equations (2.15) do not affect the aircraft dynamics and therefore are not considered, but the same logic applies
} 


\section{Dynamic Equations}

$$
\begin{aligned}
\Delta X & =m\left(\dot{u}-v_{0} \Delta r-r_{0} \Delta v+w_{0} \Delta q+q_{0} \Delta w\right) \\
\Delta Y & =m\left(\dot{v}+u_{0} \Delta r+r_{0} \Delta u-w_{0} \Delta p-p_{0} \Delta w\right) \\
\Delta Z & =m\left(\dot{w}-u_{0} \Delta q-q_{0} \Delta u+v_{0} \Delta p+p_{0} \Delta v\right) \\
\Delta L & =I_{x x} \dot{p}-I_{z x} \dot{r}+\left(I_{z z}-I_{y y}\right)\left(r_{0} \Delta q+q_{0} \Delta r\right)-I_{z x}\left(p_{0} \Delta q+q_{0} \Delta p\right) \\
\Delta M & =I_{y y} \dot{q}+\left(I_{x x}-I_{z z}\right)\left(p_{0} \Delta r+r_{0} \Delta p\right)+I_{z x}\left(2 p_{0} \Delta p-2 r_{0} \Delta r\right) \\
\Delta N & =I_{z z} \dot{r}-I_{z x} \dot{p}+\left(I_{y y}-I_{x x}\right)\left(p_{0} \Delta q+q_{0} \Delta p\right)+I_{z x}\left(q_{0} \Delta r+r_{0} \Delta q\right)
\end{aligned}
$$

\section{Kinematic Equations}

$$
\begin{aligned}
\dot{\phi} & =\Delta p+\Delta r \tan \theta_{0} \\
\dot{\theta} & =\Delta q \\
\dot{\psi} & =\Delta r \sec \theta_{0}
\end{aligned}
$$

For simplicity, we will drop the $\Delta$ - the different forces, moments and motion variables represent perturbations around their trim values, not the absolute value.

In order to avoid confusion with the subscript 0 used in the aerodynamic model, we will adopt the subscript trim for trim points.

The linear system described by equations (2.31) and (2.32) can be described in a more compact way, using state space notation:

$$
\dot{x}=A x+B u
$$

where $A$ is a square matrix known as dynamic matrix and $B$ the control matrix. Vectors $x$ and $u$ have been defined before.

There is an interesting result about the small perturbation equations, which is decoupling of longitudinal and lateral/directional motions for purely longitudinal flight conditions like straight level flight and pull-ups/push-overs. This makes the model even simpler as it separates into two lower order systems that can be studied independently. We study the case of straight level flight, which is the usual flight condition for a conventional aircraft. We will refer to the resulting model as Linear Decoupled Model.

\subsubsection{Longitudinal Equations of Motion}

Using the same formulation as in equation (2.33), longitudinal motion is described by the time history of the state vector $x=[u, w, q, \theta]$, given by equation (2.34). It is assumed that engine throttle 
is held constant and so only elevator control is considered.

$$
\begin{aligned}
\dot{x}= & {\left[\begin{array}{cccc}
\frac{X_{u}}{m} & \frac{X_{w}}{m} & -\alpha_{\text {trim }} u_{\text {trim }} & -g \cos \left(\theta_{\text {trim }}\right) \\
\frac{Z_{u}}{m-Z_{\dot{w}}} & \frac{Z_{w}}{m-Z_{\dot{w}}} & \frac{Z_{q}+m u_{\text {trim }}}{m-Z_{\dot{w}}} & \frac{-m g \sin \left(\theta_{\text {trim }}\right.}{m-Z_{\text {trim }}} \\
\frac{1}{I_{y y}}\left(M_{u}+\frac{M_{\dot{w}} Z_{u}}{m-Z_{\dot{w}}}\right. & \frac{1}{I_{y y}}\left(M_{u}+\frac{M_{\dot{w}} Z_{u}}{m-Z_{\dot{w}}}\right. & \frac{1}{I_{y y}}\left(M_{q}+\frac{M_{\dot{w}} Z_{q}+m u_{\text {trim }}}{m-Z_{\dot{w}}}\right. & \frac{-M_{\dot{w}} m g \sin \left(\theta_{\text {trim }}\right.}{I_{y y}\left(m-Z_{\dot{w}}\right)} \\
0 & 0 & 1 & 0
\end{array}\right] x } \\
& +\left[\begin{array}{c}
\frac{X_{\delta_{e}}}{m} \\
\frac{Z_{\delta_{e}}}{m-Z_{\dot{w}}} \\
\frac{M_{\delta_{e}}}{I_{y y}}+\frac{M_{\dot{w}}}{I_{y y}} \frac{Z_{\delta_{e}}}{m-Z_{\dot{w}}} \\
0
\end{array}\right] \delta_{e}
\end{aligned}
$$

Expressions for the different entries on matrices above can be found in Appendix B.

A conventional aircraft has two pairs of complex poles, corresponding to two different dynamic modes known as short period and phugoid (see Figure 2.2). Usually, they are both dynamically stable (poles on the left side of complex plane) but the case of unstable phugoid is also common.

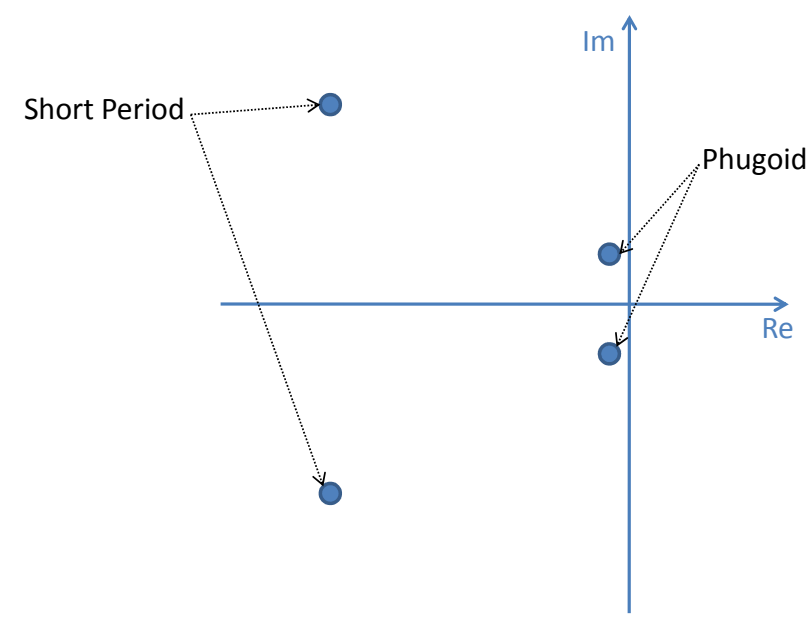

Figure 2.2: Longitudinal poles for a conventional aircraft

\subsubsection{Lateral Equations of Motion}

Using state-space notation, lateral/directional motion is described by the time history of the state vector $x=[v, p, r, \phi]$, given by: 


$$
\begin{aligned}
& \dot{x}= {\left[\begin{array}{cccc}
\frac{Y_{v}}{m} & \frac{Y_{p}}{m} & \frac{Y_{r}}{m}-u_{\text {trim }} & g \cos \left(\theta_{\text {trim }}\right) \\
\frac{L_{v}}{I_{x x}^{\prime}}+I_{z x}^{\prime} N_{v} & \frac{L_{p}}{I_{x x}^{\prime}}+I_{z x}^{\prime} N_{p} & \frac{L_{r}}{I_{x x}^{\prime}}+I_{z x}^{\prime} N_{r} & 0 \\
\frac{N_{v}}{I_{z z}^{\prime}}+I_{z x}^{\prime} L_{v} & \frac{N_{p}}{I_{z z}^{\prime}}+I_{z x}^{\prime} L_{p} & \frac{N_{r}}{I_{z z}^{\prime}}+I_{z x}^{\prime} L_{r} & 0 \\
0 & 1 & \tan \left(\theta_{\text {trim }}\right) & 0
\end{array}\right] x } \\
&+\left[\begin{array}{cc}
\frac{Y_{\delta_{a}}}{m} & \frac{Y_{\delta_{r}}}{m} \\
\frac{L_{\delta_{a}}}{I_{x x}^{\prime}}+I_{z x}^{\prime} N_{\delta_{a}} & \frac{L_{\delta_{r}}}{I_{x x}^{\prime}}+I_{z x}^{\prime} N_{\delta_{r}} \\
\frac{N_{\delta_{a}}}{I_{z z}^{\prime}}+I_{z x}^{\prime} L_{\delta_{a}} & \frac{N_{\delta_{r}}}{I_{z z}^{\prime}}+I_{z x}^{\prime} L_{\delta_{r}} \\
0 & 0
\end{array}\right]\left[\begin{array}{c}
\delta_{a} \\
\delta_{r}
\end{array}\right]
\end{aligned}
$$

A conventional aircraft has a pair of complex poles, corresponding to dutch roll, an two other real poles which associated to roll and spiral dynamic modes (see Figure 2.3). Except for spiral, which is likely to be unstable, the other two modes are usually dinamically stable.

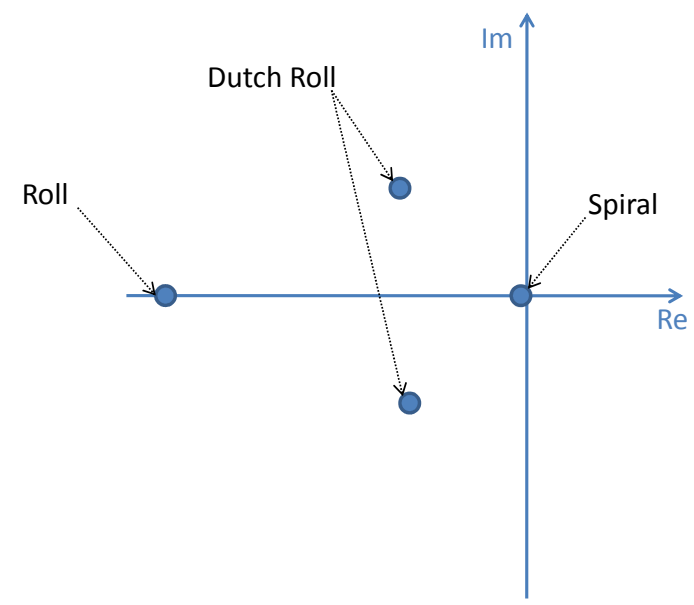

Figure 2.3: Lateral poles for a conventional aircraft

We will address to the different longitudinal and lateral dynamic modes in more detail on Chapter 3.

\subsubsection{Linearisation Algorithm}

The linearisation method of the previous section does not give any information on the magnitude of the perturbation that makes the linear approximation valid, only that it must be small. But the validity limits cannot be disregarded if we want to use the results obtained from the linear model to predict the aircraft dynamics with some acceptable level of accuracy.

Therefore, and taking advantage of today's computers, the linearisation of the model can be easily done numerically. The algorithm we follow for this work is presented below.

Consider the nonlinear system 


$$
\dot{x}=f(x, u)
$$

Making a Taylor series expansion around a point $\left(x_{e}, u_{e}\right)$ gives

$$
\dot{x}+\delta \dot{x}=f\left(x_{e}, u_{e}\right)+\frac{\partial f}{\partial x} \delta x+\frac{\partial f}{\partial u} \delta u+\text { h.o.t. }
$$

where h.o.t. stands for higher order terms.

If $\left(x_{e}, u_{e}\right)$ is an equilibrium point, then

$$
\dot{x}=f\left(x_{e}, u_{e}\right)=0
$$

Stevens and Lewis [18] present a simple method of estimating the first partial derivatives $\frac{\partial f}{\partial x}$ and $\frac{\partial f}{\partial u}$. The example given and herein reproduced uses a function with only one variable:

Consider the function $z=g(v)$. A left and a right expansion in Taylor series around a point $v=v_{e}$ gives

$$
\begin{gathered}
z_{1} \equiv g\left(v_{e}+h\right)=g\left(v_{e}\right)+h \frac{\partial g}{\partial v}\left(v_{e}\right)+h . o . t . \\
z_{-1} \equiv g\left(v_{e}-h\right)=g\left(v_{e}\right)-h \frac{\partial g}{\partial v}\left(v_{e}\right)+\text { h.o.t. }
\end{gathered}
$$

Neglecting higher order terms leads to:

$$
\left.\frac{\partial g}{\partial v}\right|_{v=v_{e}}=\frac{z_{1}-z_{-1}}{2 h}
$$

which is nothing more than the local centred derivative of the function at $v=v_{e}$, if $h$ is taken to zero.

The algorithm followed can be found in [18, pp. 202]. It starts by defining a general perturbation size for each independent variable and iteratively and individually updates it until a convergence criterion is met (see Figure 2.4.3. The outputs are the resulting $A$ and $B$ matrices, as well as linearisation validity limits. 


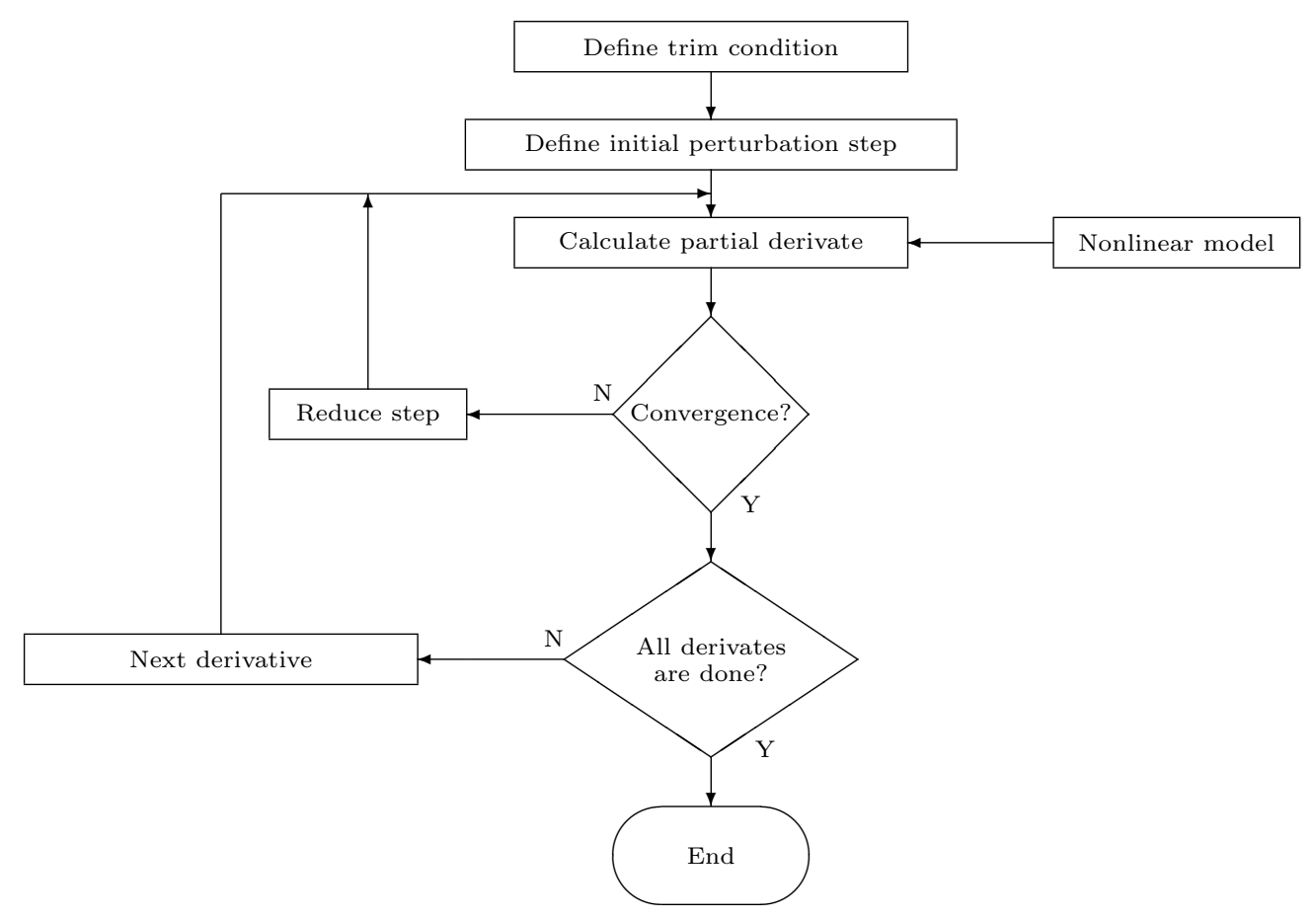

Figure 2.4: Linearisation algorithm 


\section{Chapter 3}

\section{Flying Qualities}

Flying qualities (FQ) are a set of properties that describe the ease and effectiveness with which an aircraft responds to command inputs aiming at a certain mission [4].

It is common to find in literature both flying and handling qualities, used with no distinction. Cook [4], though, distinguishes both terms. He understands flying qualities as those describing how well an aircraft performs a certain task and handling qualities as those related to the "adequacy of short term response to controls in the execution of the flight task", both from a pilot's point of view. Along this work, only the term flying qualities will be used, for simplification. We chose flying instead of handling because UAVs are the primary focus of the project and the latter might be wrongly connoted to the inevitable presence of a pilot on board. But this is only a personal interpretation; nothing similar has been found in the references consulted.

\subsection{General Overview}

Flying qualities started by being formulated in terms of pilot opinion and so they qualitatively described the aircraft dynamic behavior. In fact, pilot perception never ceased to be the main focus of flying qualities assessment, but it was found that his opinion could be substantiated by quantitative parameters describing the aircraft response. Thus, an important part of aircraft flying qualities research has been dedicated to determination of those parameters that influence pilot acceptance, [7].

The need for a numerical formulation arises from the fact that, because they play such an important role in describing the adequacy of an aircraft to the mission it was developed for, they can be used for engineering design and deficiencies identification. Quantification of flying qualities is a complex task, though.

As referred by Cook [4] flying qualities are closely related to stability and control characteristics of 
an aircraft, and therefore parameters that quantify/describe them can be used as a measure.

Stability and control elements behind flying qualities evaluation not only are related to the dynamics of the aircraft itself, but also to the interaction between pilot and aircraft, that is to say, the relation between pilot inputs (stick forces, deflections) for a certain response and the effective response of the aircraft. It "must be such that the pilot can achieve the mission objectives with reasonable physical and mental efforts" [17].

Here, we are interested in the open-loop system characteristics only. Before moving on to a flight control system, it is important to assure that we have a good starting point, meaning good open-loop dynamics. Therefore, it is possible to reduce the requirements/robustness on the closed-loop, and, consequently, the task of designing a flight control system is not as costly nor time/effort consuming.

\subsection{Requirements}

Once the importance of having good flying qualities was recognised, its demonstration became an important part of aircraft certification, without which an aircraft cannot be released into the market, either civil or military. Therefore, based on extensive, and still continuing, flight research, different requirements were created and collected in official documents emanated by airworthiness authorities, which also present an intensive test program for their demonstration. Civil and military requirements are different in nature, since the first is more safety oriented and the latter mission oriented, but without disregarding safety.

Civil requirements can be found in JARs ans FARs (Joint and Federal Aviation Regulations) or in Europe CS (Certification Specifications), like FAR23, FAR25, JARVLA, CS23 ([8]) and CS25. They are essentially qualitative and therefore rather subjective. On the other hand, military are more complete and objective, as they are established by quantitative criteria. Military requirements are laid down in military standards like Mil-F-8785C [15] and Mil-Std-1797A [14].

Flying qualities requirements, as presented by the above documents and suggested by Etkin[7], can be divided in the following different areas:

1. Control Power: it is related to the efficacy of a control in producing a range of steady equilibrium or maneuvering states.

2. Control Forces: requirements specify limits on the control forces that must be exerted by the pilot in order to effect specific changes from a given trimmed condition, or to maintain the trim speed following a sudden change in configuration or throttle setting.

3. Static Stability: it is related to the instantaneous ability of the aircraft to return to an original trim condition following a perturbation.

4. Dynamic Stability: requirement on dynamic stability are typically expressed in terms of the damping and frequency of a dynamic/natural mode. 
5. Stalling and Spinning: most requirements specify that the airplane's behavior following a stall or in a spin shall not include any dangerous characteristics, and that the controls must retain enough effectiveness to ensure a safe recovery to normal flight.

\subsection{Assessment}

Flying qualities assessment is usually made through both pilots' opinion or quantitative parameters. The first is used on flight tests and the latter on computational simulatiosn, still in the design phase, using a mathematical model describing the aircraft dynamics.

When based on pilot perception, it can be rather subjective. Therefore, a formal and clearly defined method (at least as clear as it can get) was developed to collect and interpret pilots' opinions. On flight test programs, the pilot knows the exact parameters he has to analyze and evaluate according to a set of different rating possibilities, and so reducing ambiguity. The most known and used rating scale is Cooper-Harper scale, see e.g. [4, 18, 17], which rates the results between 1 and 10, from excelent flying qualities to the existence of important deficiencies, respectively.

If using quantifiable parameters, the evaluation of flying qualities rates the results in three levels:

- Level 1: flying qualities clearly adequate for the mission flight phase

- Level 2: flying qualities adequate to accomplish the mission flight phase, but with an increase in pilot workload and, or, degradation in mission effectiveness

- Level 3: degraded flying qualities, but such that the aircraft can be controlled, inadequate mission effectiveness and high or limiting pilot workload

The boundaries between levels were set according to pilots' opinion and depend on the type/class of aircraft and flight phase/category. They are tighter on phases where more precision is required, like take-off and landing.

Mil-F-8785C [15] and Mil-Std-1797A [14] identify four classes, from I for small light airplanes, to IV for high maneuverability airplanes, and three categories, from A for non-terminal flight phases requiring rapid maneuvering, to $\mathrm{C}$ for terminal flight phases. In this report we do not go into further detail on the different phases and categories. The interested reader is referred to the previous documents.

There is a connection between both rating scales, Cooper-Harper and the three levels, which allows pilots' opinion to be supported by an analytical, and thus objective, domain. It can be found in Cook [4, pp. 257].

\subsection{UAV Flying Qualities}

UAVs are different in nature from larger/conventional aircrafts. Not considering the complex and sophisticated systems (like control systems) that usually equip UAVs, the most visible and, probably, 
important difference is the fact that there is no pilot on board. Then, there is size: today's UAVs are smaller than their piloted counterparts.

If, as mentioned in previous sections, current flying qualities requirements are founded on piloted aircraft and pilot's perception, are they still applicable to UAVs? Research on this area is quite recent and so far there are no requirements/standards laid down specifically for UAVs. There is common agreement, however, in that this is an area to invest, as recent projects and papers on this area show (see e.g. $[3,5,13]$ ).

The problem of existent requirements adequacy to UAVs is two-sided:

- FQ parameters

- FQ rating

Pilots' perception of flying qualities depends on the efforts they put into flying/controlling the aircraft, and this information is used to evaluate whether flying qualities are good or bad, that is to say, to rate flying qualities, to define the admissible limits. But for UAVs, FQ are only important if they affect mission performance and safe mission completion

Some of the parameters used to sustent pilot's opinion actually influence the aircraft behaviour in performing some task, irrespective of the fact that there is a pilot commanding the inputs and reacting to the aircraft response, improving or deteriorating it. Technically, they could be used for UAV FQ assessment. Others probably exist, though, that only make sense for piloted aircrafts, like the ones related to control force and to cue pilots.

On the other hand, it might be important, for the case of unmaned vehicles, to study parameters that had not been considered before.

As far as FQ parameters are concerned, existing studies [3, 5, 13]) focus mainly on the static and dynamic stability characteristics of the aircraf. As to FQ rating, so far it is not possible to guarantee that compliance with existing requirements will lead to good performance of the UAV, in terms of accomplishing some task/mission.

As a last note on this subject, it is important to mark that the fact that no specific requirements for UAV flying qualities have been created yet does not mean that development and assessment of operational performance of these platforms cannot be/continue to be made. Up to this point some piloted flying qualities parameters/requirements have been used.

\subsection{Choice of Flying Qualities for Analysis}

The test model for this project is a UAV and, based on what is writen above, care must be taken when choosing the parameters. Not only must they be coherent with the fact there is no pilot on board, but also have to be representative of its dynamic behaviour, since time limits our study to few 
parameters. On the other hand, it would be interesting if the results of this work, or some of them, could be useful for other aircraft types, even piloted ones, like those on the scope of CESAR Project.

But selection also has to take into account the type of information that can be taken from dynamic model of Chapter 2. Considering this first, and looking to the different flying qualities areas from Section 3.2, only 1,3 and 4 comply. These are actually the first to be addressed to in flying qualities studies, even the ones on UAVs, and most commonly found in flight mechanics/dynamics books.

Static stability, dynamic stability and control power are, then, rather important flying qualities indicators, both for piloted and nonpiloted aircrafts, and so this project will focus on them. Static stability, as defined by area 3, can be directly investigated from the sign of some stability derivatives. For a conventional aircraft, static stability requirements are usually met and therefore won't be addressed in so much detail. Instead, we will study areas 1 and 5 more deeply. Their relevance to aircraft performance is as follows:

- Control Power: it comprises static characteristics which determine the set of steady state flight conditions (already listed on Section 2.3) attainable by the aircraft, that is to say, part of the flight envelope, which shall be as wide as possible.

- Dynamic Stability: it is important to look at two different situations:

- Response after a perturbation: dynamic characteristics determine whether the aircraft tends to return to an original trim conditon or not (long term speaking, do not confuse with static stability, which refers to the instantaneous response), and also describe its response.

- Response after a control input: dynamic characteristics determine how the aircraft will respond (both in short and long term) to an intentional input aiming at a certain task, in terms of the easiness or difficulty to fulfill that task, which is directly related to the natural modes.

It is now time to look into static and dynamic characteristics in more detail, not to all the parameters they comprise, but to the most relevant ones, which we will study. Each area distinguishes between longitudinal and lateral/directional criteria and the same strategy is followed here.

\subsubsection{Longitudinal Flying Qualities}

\section{Static Characteristics}

$\delta_{e}$ vs $V$ : this term is usually known as elevator position stability or longitudinal control position stability. It gives information about the required elevator deflection to mantain straight level flight at a certain speed,also known as trim speed. The gradient of this curve is directly related to sability, if throttle command is fixed: if the gradient is positive, the aircraft is stable (for proof see Etkin [7, pp. 39]). It also boxes the trimmable speeds in terms of elevator deflection limits, but speed limits 
shouldn't be set by it. It can be estimated from equation (3.1) (expression for $\alpha$ vs $V$ is also presented, because it will be used later).

$$
\begin{gathered}
\delta_{e}=-\frac{C_{m_{0}} C_{L_{\alpha}}+C_{m_{\alpha}} C_{W}}{C_{L_{\alpha}} C_{m_{\delta_{e}}}-C_{L_{\delta_{e}}} C_{m_{\alpha}}} \\
\alpha=\frac{C_{m_{0}} C_{L_{\delta_{e}}}+C_{m_{\delta_{e}}} C_{W}}{C_{L_{\alpha}} C_{m_{\delta_{e}}}-C_{L_{\delta_{e}}} C_{m_{\alpha}}}+\alpha_{0}
\end{gathered}
$$

where $C_{W}=\frac{m g}{\bar{q} S}$ and $\alpha_{0}$ is the angle of attack for zero lift.

$\delta_{e}$ vs $g$ or $\delta_{e}$ vs $n$ : the elevator position per $g / n$ is also known as elevator position maneuvering longitudinal stability. It gives information about the extra elevator deflection to initiate (from straight level flight) and hold a steady pull-up/puch over with a load factor of $n=1+g$, at constant speed. It should have a negative gradient for maneuver stability. This curve is usually measured at constant speed (this parameter could be also studied for steady-turns). It can be estimated from the following expression [7]:

$$
\Delta \delta_{e}=-(n-1) C_{W} \frac{\left(C_{m_{\alpha}}-\frac{1}{2 \mu}\left(C_{L_{q}} C_{m_{\alpha}}-C_{L_{\alpha}} C_{m_{q}}\right)\right.}{C_{L_{\alpha}} C_{m_{\delta_{e}}}-C_{L_{\delta_{e}}} C_{m_{\alpha}}}
$$

where $\mu=\frac{2 m}{\rho S c}$.

$n$ vs $\alpha$ or $n_{\alpha}$ : the normal load factor per unit angle of attack, also known as gust sensivity or load factor sensivity, is an important parameter for short term response analysis, which measures the maneuverability of the aircraft.It can be estimated from the following expression [7], which gives the inverse relation:

$$
\Delta \alpha=\frac{n-1}{C_{L_{\alpha}}}\left(C_{W}-\frac{C_{L_{q}} C_{W}}{2 \mu}-C_{L_{\delta_{e}}} \frac{\Delta \delta_{e}}{n-1}\right)
$$

\section{Dynamic Characteristics}

Phugoid: usually, this second order-like mode (Figure 2.2 is not of much concern due to its long period nature, although an increase in damping is generally desirable. It defines the long term response after a perturbation or longitudinal control input, which consists of a motion at constant total energy, during which changes between kinetic and potencial energy occur. Therefore, as visible in Figure 3.1, the most relevant changes are in speed and altitude. Phugoid characteristics like undamped frequency $\omega_{n}$ and damping ratio $\xi$ can be estimated directly from the respective pair of poles location, as described in Appendix C. 


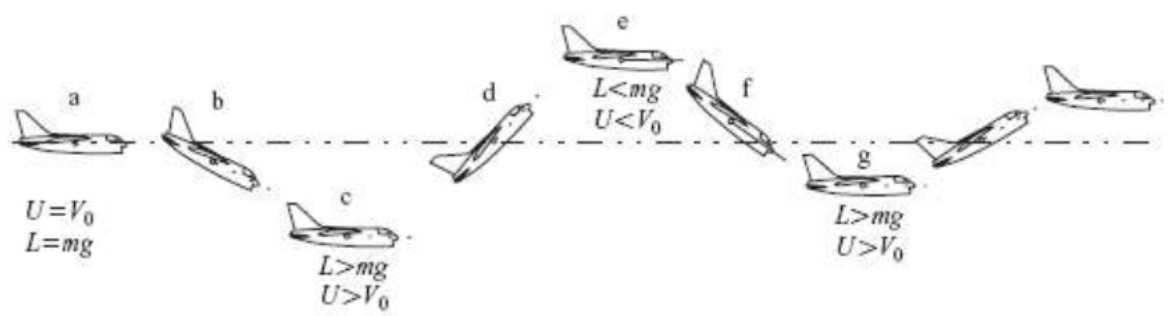

Figure 3.1: Phugoid. Reprinted from [4]

Short Period: as Figure 2.2 shows, this is a second order-like mode, usually stable and heavily damped, subsiding rapidly. It is most critical for maneuvers, because it governs the short term response. The most affected variables are angle of attack and pitch, while speed and altitude remain approximately constant, as it can be seen from Figure 3.2. Short period characteristics like undamped frequency and damping ratio can be estimated directly from the respective pair of poles location, as described in Appendix C.

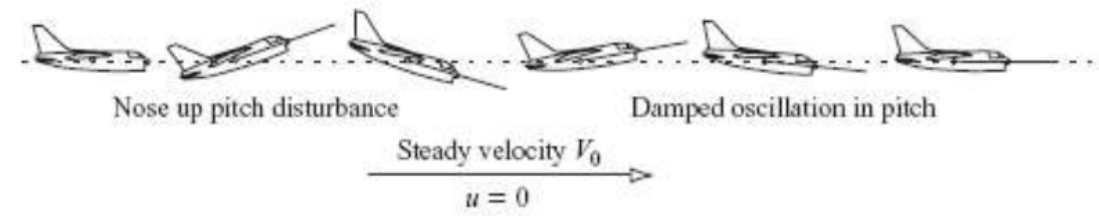

Figure 3.2: Short Period Reprinted from [4]

\subsubsection{Lateral Flying Qualities}

\section{Static Characteristics}

$\delta_{r}$ vs $\beta$ : this parameter is usually known as rudder position directional stability or directional stability, rudder-fixed. It determines the required rudder deflection to mantain steady sideslip at a certain sideslip angle $\beta$, and also boxes the trimmable sideslips in terms of rudder deflection limits $\beta$. For stability, a positive gradient is required. It can be estimated from the following expression [19]:

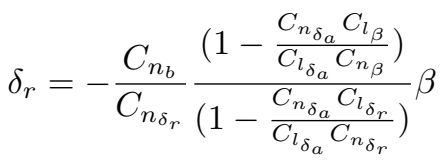

$\delta_{a}$ vs $\beta$ : this parameter is usually known as control-fixed dihedral effect. It determines the required aileron deflection to mantain steady sideslip at a certain sideslip angle. Pilot's opinion regardind this parameter indicate a negative gradient as a desirable characteristic. It can be estimated from the following expression [19]: 


$$
\delta_{a}=-\frac{C_{l_{b}}}{C_{l_{\delta_{a}}}} \frac{\left(1-\frac{C_{l_{\delta_{r}}} C_{n_{\beta}}}{C_{n_{\delta_{r}}} C_{l_{\beta}}}\right)}{\left(1-\frac{C_{n_{\delta_{a}}} C_{l_{\delta_{r}}}}{C_{l_{\delta_{a}} C_{n_{\delta_{r}}}}}\right)} \beta
$$

$\phi$ vs $\beta$ : this parameter is usually known as sideforce characteristic.It represents the variation of roll angle with sideslip, in a steady sideslip conditon. It is specially important for piloted aircrafts, since it cues the pilot on a nonzero sideslip. Though it might not be so relevant for UAVs, it is interesting to see how much bank (roll angle) is required to maintain steady sideslip. Usually, a positive gradient is desirable. It can be estimated from the following expression [19]:

$$
\phi=-\frac{1}{C_{L}}\left(C_{Y_{\beta}} \beta+C_{Y_{\delta_{r}}} \delta_{r}\right)
$$

\section{Dynamic Characteristics}

Roll: Figure 2.3 shows that this is usually a first order-like, very quick response. It is deliberately excited, through an aileron input, to make changes in roll angle, required for turns, for example. The important parameters describing this mode are are time constant $\tau$ and time to reach a certain roll angle (we will consider time to reach $\phi=60^{\circ},\left.t\right|_{\phi=60^{\circ}}$ ), which can be determined as described in Appendix C. The aircraft motion associated to roll mode is represented in Figure 3.3.

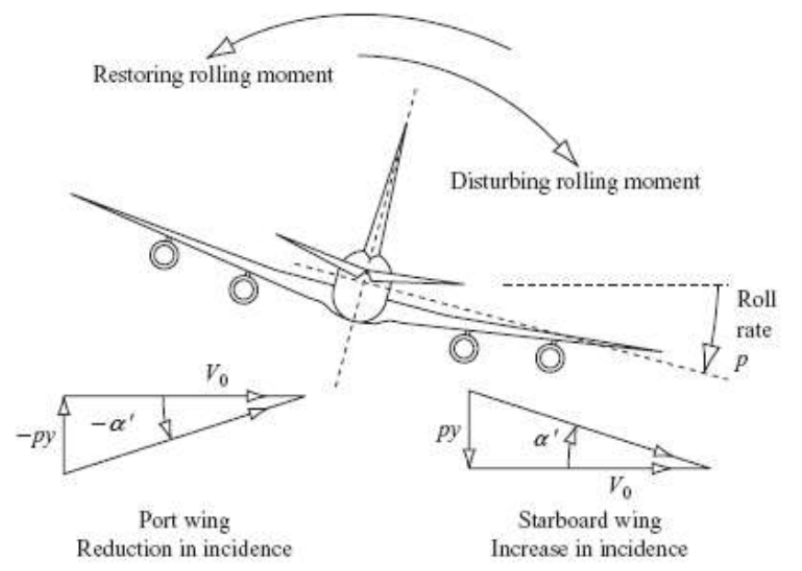

Figure 3.3: Roll. Reprinted from [4]

Spiral: Figure 2.3 shows that this is usually a first order-like mode, with a quite large time constant, as it can be seen from the close proximity of the pole to the origin. It is usually excited following a disturbance in roll angle, which is also the most affected variable. In conventional aircraft, spiral can be either convergent or divergent, or even neutrally stable. Because it takes so much time to develop, it might be difficult to perceive it. The unstable case is the one requiring more attention, for logical reasons. Actually, because of its typical large time constant, unstability can be acceptable 
if the time to double amplitude $T_{2}$ is over a certain limit Appendix $\mathrm{C}$ shows how to determine this parameter. Figure 3.4 represents the aircraft complex motion associated with this mode, involving coupling between roll, yaw and sideslip.
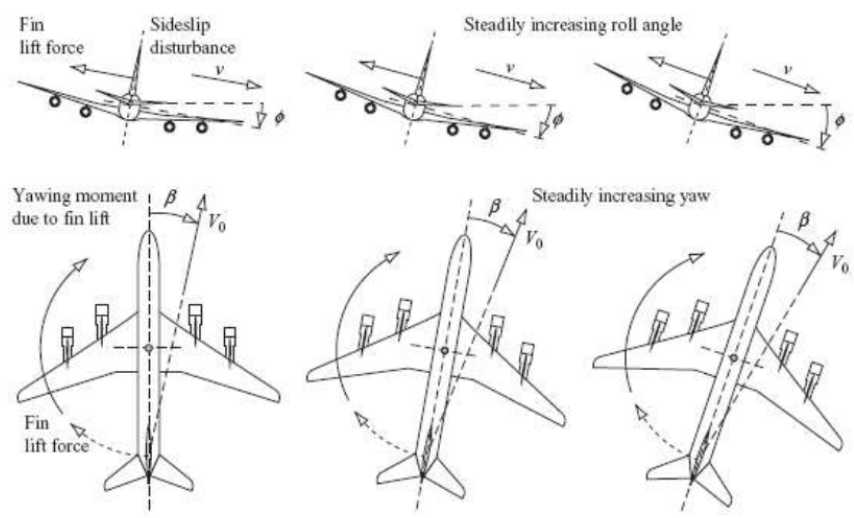

Figure 3.4: Spiral. Reprinted from [4]

Dutch Roll: this mode is not usually excited deliberately. Figure 2.3 shows that this is a secondorder-like damped mode, the lateral equivalent to short period. The resultant motion (Figure 3.5) involves yawing, rolling and sideslipping. Dutch roll characteristics like undamped frequency and damping ratio can be estimated directly from the respective pair of poles location, as described in Appendix C.

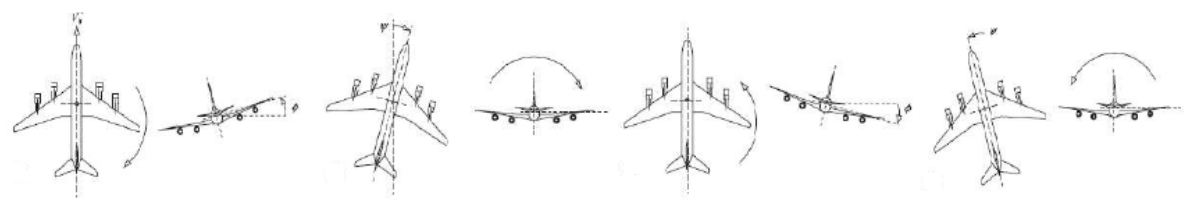

Figure 3.5: Dutch Roll. Reprinted from [4]

\subsubsection{Parameter Determination}

Military standards and civil regulations for have dedicated sections to flight test procedures for criteria demonstration of compliance. At a design stage, flight testing is not possible and FQ evaluation depends on computational simulation, that shall follow flight test practice as much as possible.

Last section indicated how the different $\mathrm{FQ}$ parameters could be determined through a linear approximation, but they can also be directly extracted from the full nonlinear model. The objective of this section is to define the simulation inputs and baseline fligh condition to do this. As far as dynamic characteristics are concerned, the ability to study each mode independently depends on how 
well separated they are from each other in terms of natural frequencies. Otherwise, coupling might occur and difficult the analysis.

Tables 3.1 and 3.2 show how to extract the different static and dynamic FQ parameters from the nonlinear model.

Usually, longitudinal modes are well separated in frequency and so they can be easily identified in the longitudinal response of the aircraft, no matter the input form used. Unlike the logitudinal case, there is a tendency for coupling between lateral modes and therefore is is not an easy task to excite them independentely.

\begin{tabular}{|l|l|l|}
\hline Static Characteristic & Trim Condition & Observations \\
\hline \hline$\delta_{e}$ vs $V$ & Straight Level & \\
\hline$\delta_{e}$ vs $g$ & Steady Pull-up & At the same speed \\
$n_{\alpha}$ & & \\
\hline$\delta_{r}$ vs $\beta$ & Steady Sideslip & Constant heading \\
$\delta_{a}$ vs $\beta$ & & \\
$\phi$ vs $\beta$ & & \\
\hline
\end{tabular}

Table 3.1: Static flying qualities parameters

\begin{tabular}{|c|c|c|c|}
\hline Mode & Mode Excitation & Trim Condition & Observations \\
\hline Phugoid & Elevator Step & \multirow{5}{*}{ Straight Level } & \\
\hline $\begin{array}{l}\text { Short } \\
\text { Period }\end{array}$ & Elevator Doublet & & $\begin{array}{l}\text { Short duration doublet } \\
\text { used to avoid phugoid } \\
\text { excitation }\end{array}$ \\
\hline Spiral & Rudder Pulse & & $\begin{array}{l}\text { After the pulse, } \phi \\
\text { disturbance should be } \\
\text { equal to } 20^{\circ}\end{array}$ \\
\hline Roll & Aileron Step & & $\begin{array}{l}\text { In flight tests, short } \\
\text { duration pulse and } \\
\text { steady turn are used } \\
\text { to avoid large excur- } \\
\text { sions in } \phi\end{array}$ \\
\hline $\begin{array}{l}\text { Dutch } \\
\text { Roll }\end{array}$ & Rudder Doublet & & $\begin{array}{l}\text { Doublet period similar } \\
\text { to mode period }\end{array}$ \\
\hline
\end{tabular}

Table 3.2: Dynamic flying qualities parameters 


\begin{tabular}{|l|l|}
\hline \multicolumn{2}{|c|}{ Static Characteristics } \\
\hline \hline \multicolumn{2}{|c|}{ Longitudinal } \\
\hline$\delta_{e}$ vs $V$ & positive gradient \\
$\delta_{e}$ vs $g$ & negative gradient \\
$n_{\alpha}$ & not specified \\
\hline \multicolumn{2}{|c|}{ Lateral } \\
\hline$\delta_{r}$ vs $\beta$ & positive gradient \\
$\delta_{a}$ vs $\beta$ & negative gradient \\
$\phi$ vs $\beta$ & positive gradient \\
\hline
\end{tabular}

\begin{tabular}{|c|c|}
\hline \multicolumn{2}{|c|}{ Dynamic Characteristics } \\
\hline \multicolumn{2}{|c|}{ Longitudinal } \\
\hline$\omega_{n_{p h}}$ & not specified \\
\hline$\xi_{p h}$ & above a certain value \\
\hline $\begin{array}{l}\omega_{n_{s p}} \\
\xi_{s p}\end{array}$ & within certain limits \\
\hline \multicolumn{2}{|c|}{ Lateral } \\
\hline $\begin{array}{l}\omega_{n_{d r}} \\
\xi_{d r}\end{array}$ & above a certain value \\
\hline$T_{2_{\text {spiral }}}$ & above a certain value \\
\hline $\begin{array}{l}\tau_{\text {roll }} \\
\left.t\right|_{p h i=60^{\circ}}\end{array}$ & below a certain value \\
\hline
\end{tabular}

Table 3.3: Flying qualities requirements

\subsubsection{Typical Requirements}

Although some of the desired characteristics for the different flying qualities parameters have been mentioned in the last subsection, it is pertinent to list them all in a more organised way for further consult. Table 3.3 shows typical requirements, in a qualitative way. 


\section{Chapter 4}

\section{ANTEX-M X03 Flying Qualities}

All following studies use PoAF UAV ANTEX-M X03 (or simply ANTEX) as the test model and therefore we need its dynamic model.

According to deficiencies identifyied in existing data (see Appendix A), some model parameters need to be determined/recalculated. Then, following the procedure of Chapter 2, it is possible to build the 6-DOF dynamic model. Before doing parametric studies, the baseline characteristics of the UAV must be computed and that is what this chapter is about: building the dynamic model and using it for assessment of current flying qualities.

\subsection{Dynamic Model}

Chapter 2 presented the general form for an aircraft 6-DOF dynamic model. In order to apply it to a specific aircraft, in this case a UAV, its mass properties, aerodynamic characteristics and propulsion system must be known. These parameters are determined in this section, and, together with equations 2.13 and 2.14, they completely define the dynamic model. Only model parameters are presented, not the complete nonlinear system.

\subsubsection{Mass Properties}

The mass properties required for a dynamic model are the CG position and the inertia matrix. Due to the complex geometry and dimension of the aircraft, as well as the multiplicity of materials it is made of, inertia matrix determination is quite difficult and might take some time. This can be done either experimentally or computationally.

Félix and Saraiva [9] tried to determine ANTEX inertia matrix using an experimental method, which required the construction of a structure where the aircraft would be suspended as a pendu- 
lum. By making it rotate around different axes, an analysis of its periodic motion would allow the determination of the inertia moments (but not the inertia products). Nevertheless, experiments were not successful, since results validation using a beam with the same weight of the aircraft failed. The structure built for this purpose, which experienced large deformation during the tests, was pointed out as one of the most probable causes.

Another way to determine the mass properties experimentally is through flight tests. However, none of the two experimental methods would be feasible within the working time of this project.

The solution is, then, to determine the inertia matrix computationally. There is a 3D CAD model of the UAV already available.

\subsubsection{CG Position}

Before measuring the inertia matrix, the CG position must be known. Accordind to Félix and Saraiva [9] , the CG lies on the point defined in Table 4.1.

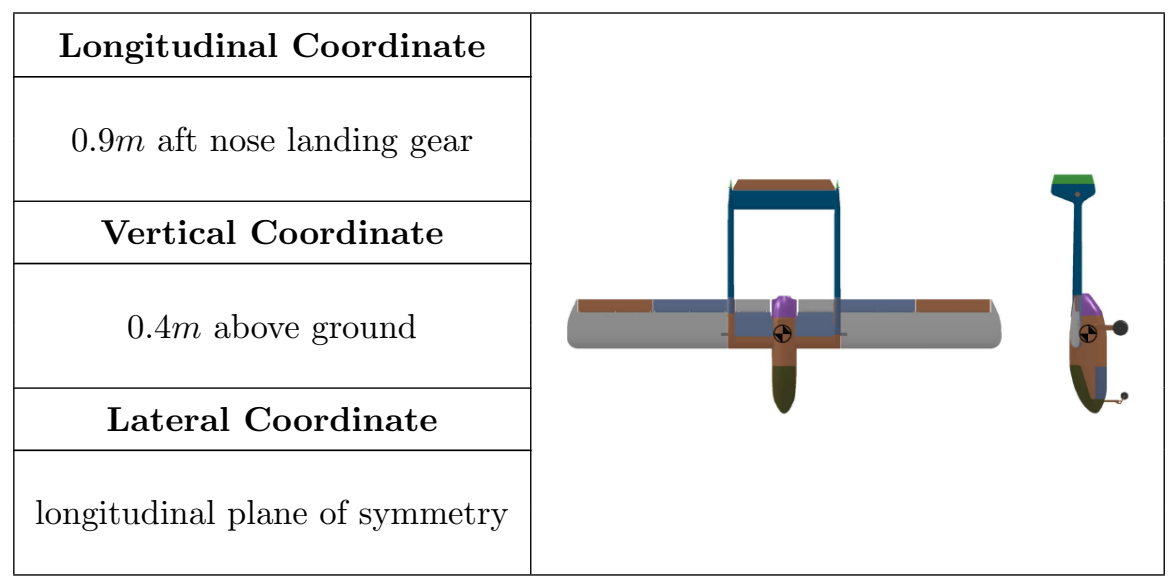

Table 4.1: CG position

Both longitudinal and vertical coordinates were determined experimentally, the first by measuring the reactions on the landing gear and performing a torque balance, and the second with the aid of a plumb ${ }^{1}$. Conversely, the lateral position is a consequence of the assumption that the airplane is longitudinally symmetric.

Although it was designed to be symmetric, it is always difficult to guarantee that no asymmetries arise during construction, especially on a handmade aircraft. In fact, by individually weighting each wing, a small difference was noticed (about $250 \mathrm{~g}$ ), and this is only one example. Nevertheless, these effects are usually small and can be neglected. These values were determined for a configuration without fuel, avionics and payload. We will assume that they can be accommodated in a way that the CG does not shift significantly away from the already calculated position.

For this work, we recalculated CG coordinates under the same conditions, and using the torque

\footnotetext{
${ }^{1}$ Because of the imprecision inherent to these experiments, only the first decimal place is presented.
} 
balance method for both longitudinal and vertical position. New values agreed with the previous ones.

\subsubsection{Inertia Matrix}

We use SolidWorks, 3D CAD design software,to determine the inertia matrix. Among other things, it allows the introduction of information on the various materials used.

The existing SolidWorks 3D model was built to a purpose other than mass properties determination. In order to compute the inertia matrix, we need to make several modifications that demand quite some time. Specification of all the materials used in the aircraft is not feasible, because there is no accurate record of it. Taking both these facts into consideration, only the main components are accounted for. As a result, the values obtained are approximated. The strategy adopted tries to assure that they are reasonable, though.

We compute the inertia matrix by summing up the different contributions of the main components. For the approximation to be good, these components must be representative of the whole aircraft and its general mass distribution. The components considered are:

- Main Structure

- Wings

- Remaining

- Payload and Avionics

- Engine and Fuel

- Propeller

- Ballast

Figure 4.1 shows the SolidWorks model used. Contribution from the last two items is determined separately for simplicity, and that is why they are not part of the model.

UAV skin is not included because it would take too much time to model computationally. It might seem like significative errors can arise from this decision, but, for inertia purposes, the structure is more important. This does not mean that its mass is neglected - in the end, the total mass of the model must be the same of the aircraft. There are certainly errors, for this is an approximation, but to make the project feasible trade-offs are required.

The results obtained are:

SolidWorks: Main structure, payload and avionics, engine and fuel

$$
I_{\text {main }}=\left[\begin{array}{ccc}
81 & 0.0145 & 2.665 \\
0.0145 & 22.74 & -0.0243 \\
2.665 & -0.0243 & 97.19
\end{array}\right]
$$




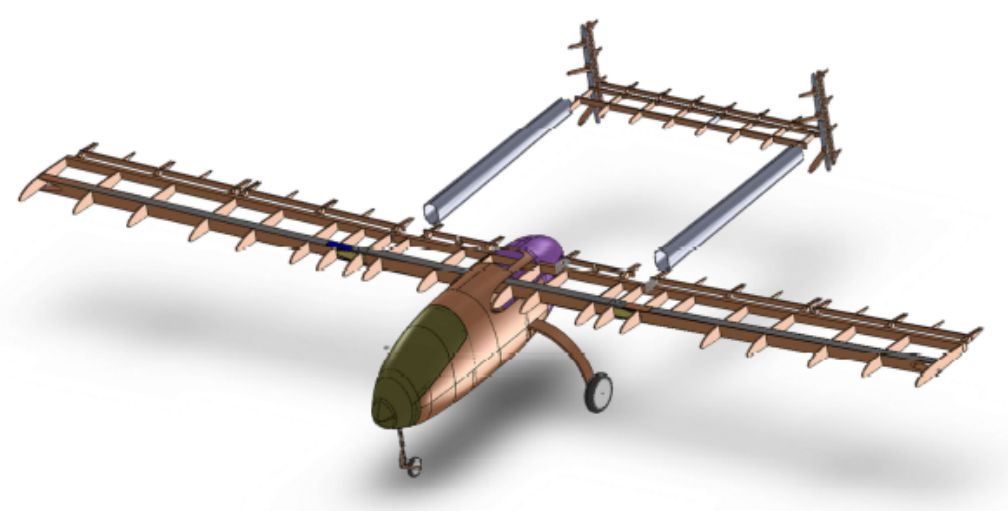

Figure 4.1: SolidWorks model used for inertia matrix computation

A first glance to the above matrix shows that $I_{x y}$ and $I_{y z}$ don't vanish, contrary to what would be expected for longitudinal symmetry. However, comparing the order of magnitude of the different entries, the inertia products relative to the lateral axis can be neglected, and it can be assumed, with a considerable level of accuracy, that the aircraft is indeed longitudinally symmetric.

\section{Propeller and Ballast:}

Because the propeller has small mass $(350 \mathrm{~g})$ and is close to the CG, its contribution can be neglected. The same is not valid for the ballast, which is heavier and located significantly far from the CG. It can be assumed that it consists of a point mass located in the nose of the UAV. Therefore, its contribution can be determined from the Parallel Axis Theorem ( information on this theorem can be found in reference [1]. If we further assume that the point mass lies on the longitudinal axis, then the resultant products of inertia are zero. Considering the above, ballast contribution to inertia matrix is:

$$
I_{\text {ball }}=\left[\begin{array}{ccc}
0 & 0 & 0 \\
0 & 15 & 0 \\
0 & 0 & 15
\end{array}\right]
$$

\section{Final Inertia Matrix:}

Summing up the different contributions leads to:

$$
I=\left[\begin{array}{ccc}
81 & 0 & 2.67 \\
& 37.74 & \\
2.67 & & 112.19
\end{array}\right]
$$

which we use as the inertia matrix for the whole aircraft. 


\subsubsection{Aerodynamic Model}

This subject has already been explored on Chapter 2. Here, we present the results obtained for ANTEX and compare them to the existing ones.

Even with some results already available, it is important to repeat the analysis, not only because there are some derivatives missing, but also for the uncertainties involving some of them, especially the ones relative to angular velocity (also known as dynamic derivatives) and lateral control surfaces (rudder and aileron), as remarked in [9].

We use CFD software EDGE for this purpose. Because a lot of simulations need to be done, some demanding considerable computational effort, and because the objective is to validate existing results, viscous effects are not accounted for, except for drag prediction and stall detection. This greatly reduces the time required for the analysis, and results are quite reasonable.

Tables 4.2 and 4.3 list both new and old results, for longitudinal and lateral derivatives, respectively. Results predicted by empiric expressions from Appendix B are also shown, as they give cues about the expected order of magnitude and sign for each of the derivatives (another pertinent form of validation that will be very useful for parametric studies). These expressions result from the sum of contributions from tail, wings and body. The last two are usually more difficult to compute and some of the results in Tables 4.2 and 4.3 only account for tail contribution. We look through empiric expressions in more detail on Chapter 5 .

\section{Longitudinal}

\begin{tabular}{|l|rr|rl|}
\hline Deriv. & EDGE & Existing & Empiric & Observations \\
\hline \hline$C_{L_{\alpha}}$ & 5.35 & 5.20 & 5.46 & \\
$C_{m_{\alpha}}$ & -0.80 & -0.58 & 1.35 & \\
\hline$C_{L_{q}}$ & 8.72 & & 2.72 & Tail contribution \\
$C_{m_{q}}$ & -10.32 & & -7.07 & Tail contribution \\
\hline$C_{L_{\dot{a}}}$ & & & -2.02 & Tail contribution \\
$C_{m_{\dot{a}}}$ & & & -7.07 & \\
\hline$C_{L_{\delta_{e}}}$ & 0.47 & 0.38 & 0.30 & \\
$C_{m_{\delta_{e}}}$ & -1.09 & -0.91 & -0.77 & \\
\hline
\end{tabular}

Table 4.2: Longitudinal aerodynamic derivatives

Table-4.2 shows good agreement between new and and existing values, with EDGE results being slightly higher (probably a consequence from not considering viscosity).

Existing values for $q$ derivatives fall outside the expected/usual interval and are not presented. As to the angle of attack rate derivatives, we didn't compute them through CFD. Therefore, $C_{L_{\dot{a}}}$ will be 
considered zero for further analysis ${ }^{2}$. The consequences of doing this will be analysed on Chapter 5 . $C_{m_{\dot{a}}}$ will take the value predicted by the respective empiric expression.

Lift and pitching moment do not vanish for zero angle of attack. Therefore, these values are also required (we use the existing ones):

$$
\begin{aligned}
C_{L_{0}} & =0.091 \\
C_{m_{0}} & =-0.037
\end{aligned}
$$

Results for drag (existing ones) are presented separately, because, contrary to the others, which are linear in the considered flight envelope, drag is highly nonlinear, as Figures 4.2 and 4.3 reveal.

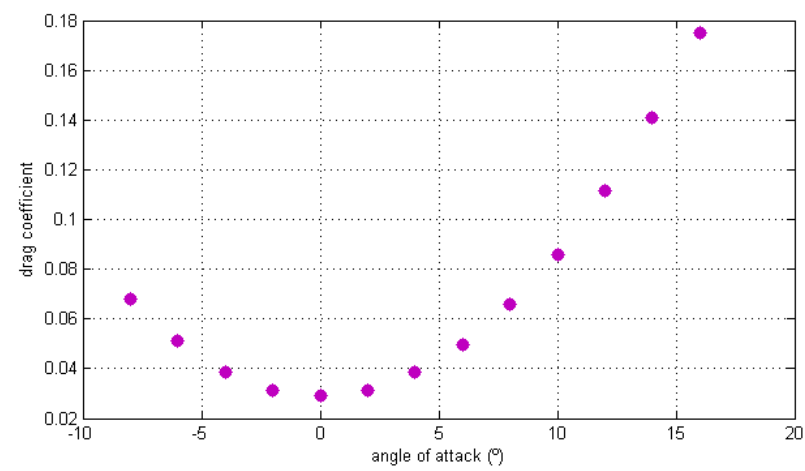

Figure 4.2: Drag variation due to angle of atack

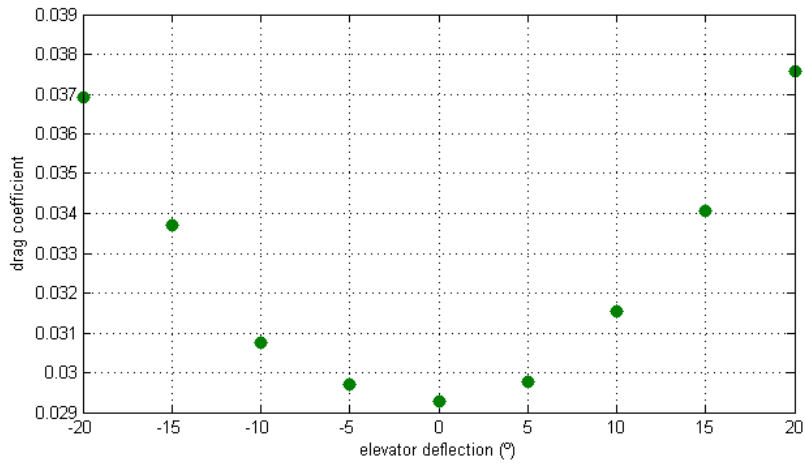

Figure 4.3: Drag variation due to elevator deflection

Both drag due to angle of attack and drag due to elevator deflection are well interpolated by parabolic curves. Total drag can then be computed from the following expression:

\footnotetext{
${ }^{2}$ We do this approximation for simplicity and because, generally, $C_{L_{\dot{a}}}$ is not very relevant for the type of results we want to compute.
} 


$$
\begin{aligned}
C_{D} & =C_{D_{0}}+f(\alpha)+g\left(\delta_{e}\right) \\
& =0.0295+0.0006 \alpha^{2}-0.0001 \alpha+0.00002 \delta_{e}^{2}+0.0000173 \delta_{e}
\end{aligned}
$$

where $\alpha$ and $\delta_{e}$ have units of degree.

Although the aerodynamic model derived in Section 2.1.3 is linear, equation 4.1, which is more accurate, will be used for total drag prediction. However, the longitudinal linear model from equation 2.34 requires drag derivatives $C_{D_{\alpha}}$ and $C_{D_{\delta_{e}}}$ and, in this case, the concept of local derivatives is applied.

\section{Lateral}

\begin{tabular}{|l|rr|rl|}
\hline Deriv. & EDGE & Existing & Empiric & Observations \\
\hline \hline$C_{Y_{\beta}}$ & -0.31 & -0.40 & -0.22 & Tail contribution \\
$C_{l_{\beta}}$ & -0.03 & -0.043 & -0.01 & Tail contribution \\
$C_{n_{\beta}}$ & 0.0439 & 0.0435 & 0.07 & Tail contribution; body's is negative \\
\hline$C_{Y_{p}}$ & -0.1015 & -0.0844 & -0.0243 & Tail contribution \\
$C_{l_{p}}$ & -0.5759 & -0.609 & 0.0023 & Tail contribution, usually negligible \\
$C_{n_{p}}$ & -0.0066 & -0.0059 & 0.0077 & Tail contribution; wing's is negative \\
\hline$C_{Y_{r}}$ & 0.1689 & 1.659 & 0.1388 & \\
$C_{l_{r}}$ & 0.0404 & 0.187 & 0.0077 & Tail contribution \\
$C_{n_{r}}$ & -0.0433 & -0.247 & -0.0438 & \\
\hline$C_{Y_{\delta_{a}}}$ & -0.0342 & 0.0246 & & Usually negligible \\
$C_{l_{\delta_{a}}}$ & -0.289 & & -0.388 & \\
$C_{n_{\delta_{a}}}$ & 0.0032 & 0.1312 & 0.006 & \\
\hline$C_{Y_{\delta_{r}}}$ & 0.163 & 0.126 & 0.1727 & \\
$C_{l_{\delta_{r}}}$ & 0.0141 & 0.0046 & 0.0095 & \\
$C_{n_{\delta_{r}}}$ & -0.0523 & 0.040 & -0.0545 & \\
\hline
\end{tabular}

Table 4.3: Lateral stability derivatives

Table 4.3 shows close proximity between the different results, for most of the derivatives. Significant discrepancies are found, however, on $C_{Y_{r}}, C_{n_{\delta_{a}}}$ and $C_{l_{\delta_{r}}}$. Comparison with empiric values indicates that the new values for these derivatives are probably more reliable.

\subsubsection{Final Aerodynamic Model}

From the above analysis, we can conclude that:

- Existing $\alpha$ and $\beta$ derivatives, as well as $\delta_{e}$ derivatives, can be considered valid 
- Comparison with empiric values indicates new $p$ and $r$ derivatives as more reliable

- For $q$ derivatives, empiric values should be used

- Comparison with empiric values indicates new rudder and aileron derivatives as more reliable

- Derivatives where uncertainty is bigger, comparing the three values, are: $C_{m_{\alpha}}, C_{L_{q}}, C_{L_{\dot{\alpha}}}, C_{m_{\dot{\alpha}}}$, $C_{l_{r}}, C_{Y_{\delta_{a}}}, C_{n_{\delta_{a}}}$

In spite of all the validation work done to reduce uncertainties in first order derivatives, they are still there, specially on the derivatives of last item. It is important to analyse how this can affect the parameters to extract from the model, and this problem will be addressed to on Chapter 5 .

Taking the above into consideration, the aerodynamic model will be built over the following derivatives:

- $\alpha, \beta$ and $\delta_{e}$ derivatives: existing values

- $C_{L_{\dot{\alpha}}}$ is considered zero

- $C_{m_{\dot{\alpha}}}$ : empiric value

- remaining: new values

\subsubsection{Some Important Results}

Some conclusions on aircraft static stability can be drawn just by looking to the aerodynamic model, in particular to stability derivatives. Stability requirements are based on the usually called stiffness derivatives and damping derivatives, which express the aircraft natural ability to resist perturbations in aerodynamic angles and angular velocity, respectively. These requirements can be found on Table 4.4. According to them and looking to previous results, we conclude that ANTEX is statically stable. Usually, for conventional aircraft this is a design requisite.

\begin{tabular}{|c|c|}
\hline Derivative & Requirement \\
\hline \hline$C_{m_{\alpha}}$ & $<0$ \\
$C_{m_{q}}$ & $<0$ \\
$C_{l_{\beta}}$ & $<0$ \\
$C_{n_{\beta}}$ & $>0$ \\
$C_{l_{p}}$ & $<0$ \\
$C_{n_{\beta}}$ & $<0$ \\
\hline
\end{tabular}

Table 4.4: Requirements for static stability

An important result that is not predicted by the aerodynamic model here derived is the stallangle, that imposes a maximum limit for angle of attack. According to EDGE simulation, $\alpha_{\max }=16^{\circ}$. 


\subsubsection{Propulsion System}

ANTEX is equipped with a propeller single-piston engine, working at constant break horse power. A general model for this kind of propulsion system can be found in Coutinho [6]. It is not relevant to reproduce it here.

\subsection{Flying Qualities Assessment}

The parameters listed on Chapter 3, Section 3.5, are now studied for ANTEX. Because the static characteristics are easier to obtain from the nonlinear model, they are presented first.

\subsubsection{Static Characteristics}

The static characteristics from Table 3.1 result from specific sets of trim conditions, all along the flight envelope. Not only do they describe the aircraft performance inside the envelope but also define some of its limits ${ }^{3}$.

Running the trim algorithm for a specific flight condition makes static characteristics determination quite straightforward, if there is a good initial guess. Otherwise, convergence can be difficult or even not possible. Therefore, after describing the steady-state flight condition of interest, results from linear model based expressions (see equations 3.1 to 3.7 ) are used as initial guesses.

Figures 4.4 to 4.6 present the different results obtained with both the nonlinear model ${ }^{4}$ and linear model based expressions.

Before looking at the static characteristics curves, it is important to check the convergence error (from the trim algorithm), since it is a measure of how reliable the nonlinear results are. In general, the error is very small, but there are three peaks in pull-up convergence error curve exceeding the convergence criterion from Section $2.3(C<0.001)$. Still, this only happens for specific and few values of $n$ and, therefore, we consider the results as acceptable ${ }^{5}$.

For all the static characteristics studied, there is close proximity between linear and nonlinear results, indicating that equations 3.1 to 3.7 can be used, with good level of accuracy, for their prediction. However,in the roll angle curve it is possible to see that, as sideslip angle increases, the two lines tend to diverge, indicating a limit for linear validity.

If the equilibrium points are stable, then, this proximity between linear and nonlinear results is expectable: for small perturbations around the trim condition, in a Taylor series expansion, neglecting second and higher order terms usually leads to quite acceptable approximations.

\footnotetext{
${ }^{3}$ Other characteristics that are beyond this study (e.g.: aeroelasticity) also have to be taken into account when determining the flight envelope.

${ }^{4}$ Nonlinear model results are obtained by repeatedly running the trim algorithm for a flight condition, varying either speed, load factor or sideslip angle.

${ }^{5}$ This error could be reduced by increasing the maximum number of iterations of fminsearch, but the computation time would also increase.
} 


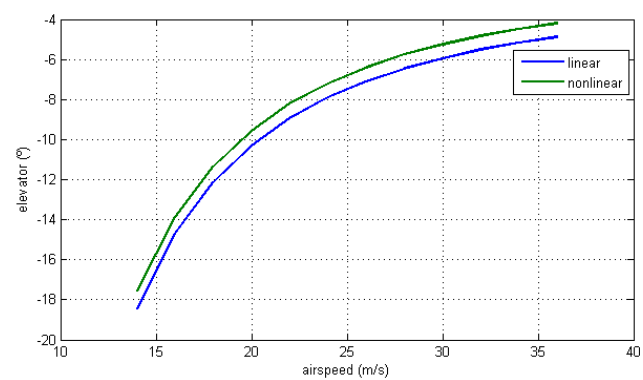

(a) Elevator versus speed

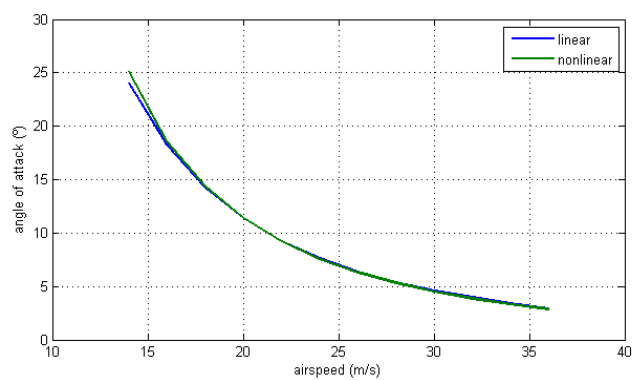

(b) Angle of attack versus speed

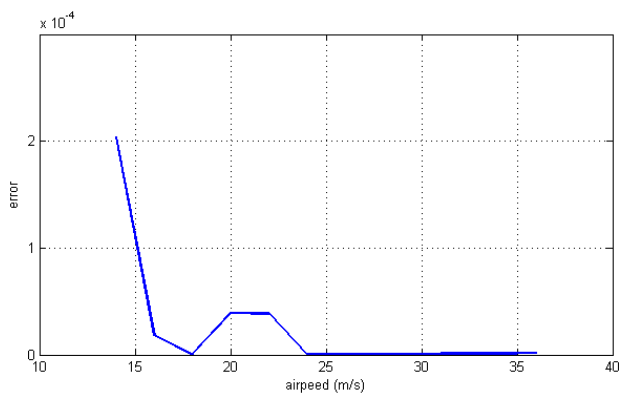

(c) Convergence error

Figure 4.4: Longitudinal static characteristics in straight level flight

Regardind now the physical meaning of the different curves, they all have the required slope for a stable aircraft, according to Table 3.3. In fact, this is an expected result: as equations 3.1 to 3.7 show, these curves depend on some of the stiffness and damping derivatives which had already shown that ANTEX-M X03 is statically stable.

But not only the slope requires attention: stall and control surfaces deflection limits must also be investigated.

By defining the maximum angle of attack, stall also sets the limit for minimum speed. CFD simulations indicated a stall angle of $16^{\circ}$ and, looking at Figure $4.4(\mathrm{~b})$, the minimum speed at which the UAV can fly is approximately $18(\mathrm{~m} / \mathrm{s})$. Following the same logic, and considering now the curve $\alpha$ vs $n$, for a speed of $30 \mathrm{~m} / \mathrm{s}$ (see Figure 4.5(b)), pull-ups with a load factor above 3 shall be avoided. To overcome this, the starting speed for the maneuver can be increased, but not above $36 \mathrm{~m} / \mathrm{s}$, which, according to Appendix A, is the maximum speed.

These limits shall never be imposed by maximum/minimum control surfaces deflection, otherwhise aircraft potencial might be underused. Considering that control surfaces can be deflected up to $\pm 15^{\circ}$, this problem does not arise, though inspection of Figure 4.4(a) shows that margin for low speed is quite narrow. This is definitely something worth improving.

The same does not happen with lateral characteristics, since, for a maximum sideslip angle of $10^{\circ}$, rudder and aileron required deflections are well inside their mechanical limits. 


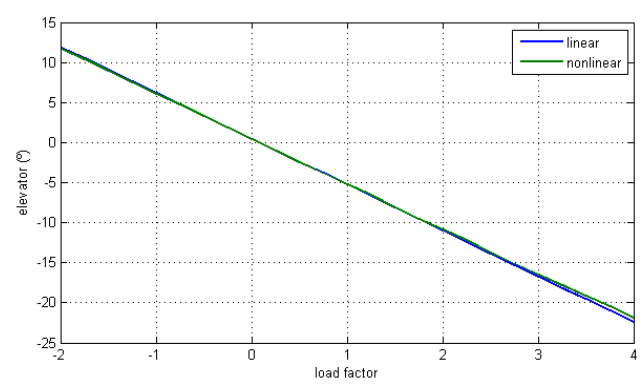

(a) Elevator versus load factor

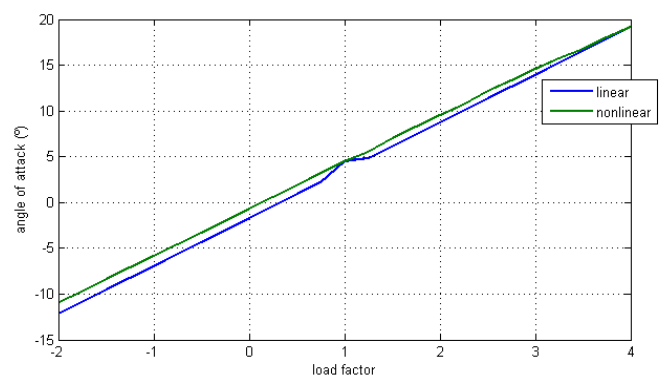

(b) Angle of attack versus load factor

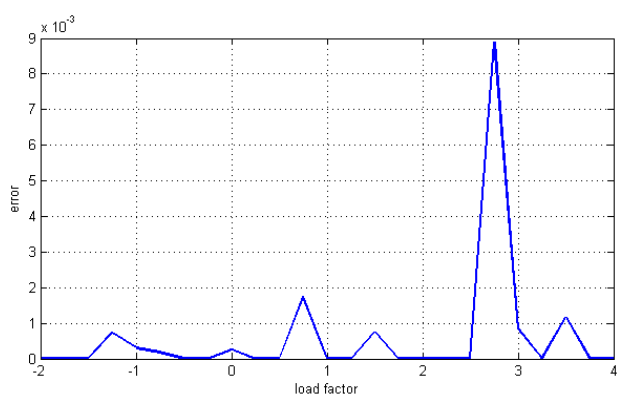

(c) Convergence error

Figure 4.5: Longitudinal static characteristics in pull-up/push-over

\subsubsection{Dynamic Characteristics}

The easiest and most common way to study the dynamic parameters from Section 3.5 is through a linear model: a lot of interesting and intuitive characteristics, which explain the nonlinear behaviour of the aircraft, giving it a physical meaning, can be determined just by looking to the poles of the system. Additionally, dynamic characteristics parameters from Table 3.3 are linear model-based parameters. Nevertheless, it is possible (and also important) to extract them from the nonlinear model.

The approach followed in this case, as well as the way results are presented, are different from the ones used for static characteristics, because dynamic behaviour is more complex.

\subsubsection{Linear response}

The baseline flight condition for the study is straight level flight at cruise speed ${ }^{6}, \simeq 30 \mathrm{~m} / \mathrm{s}$. The first thing to do, then, is to define this condition. After running the trim algorithm for straight level

\footnotetext{
${ }^{6}$ This is the most interesting flight condition to study because, technically, it is the one at which more flight time is spent.
} 


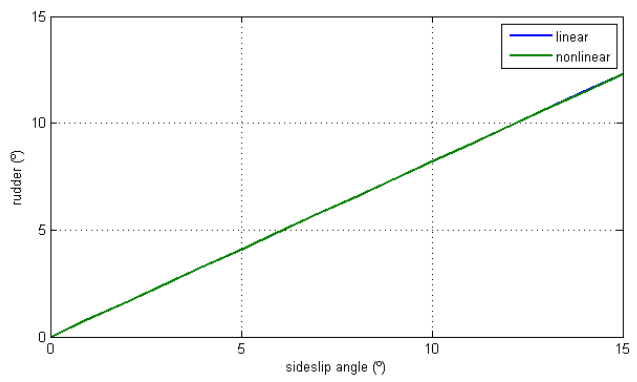

(a) Rudder versus sideslip angle

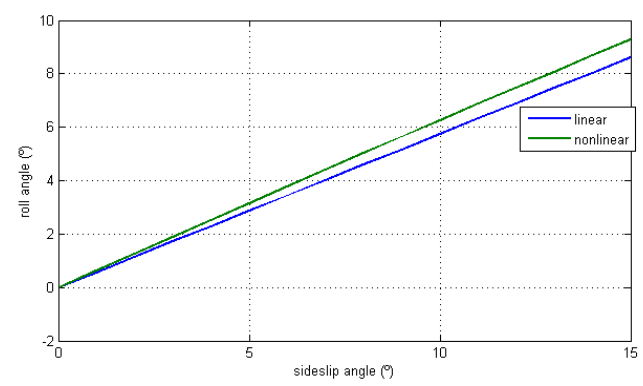

(c) Roll angle versus sideslip angle

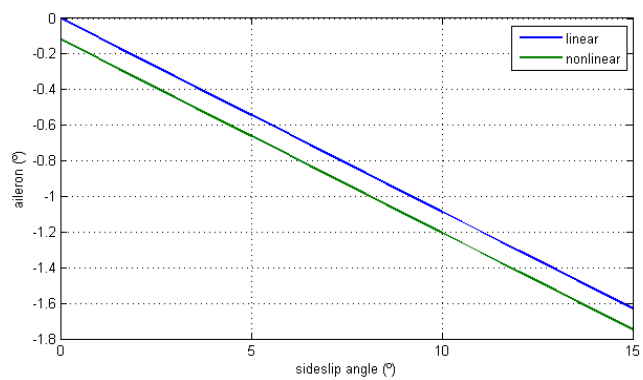

(b) Aileron versus sideslip angle

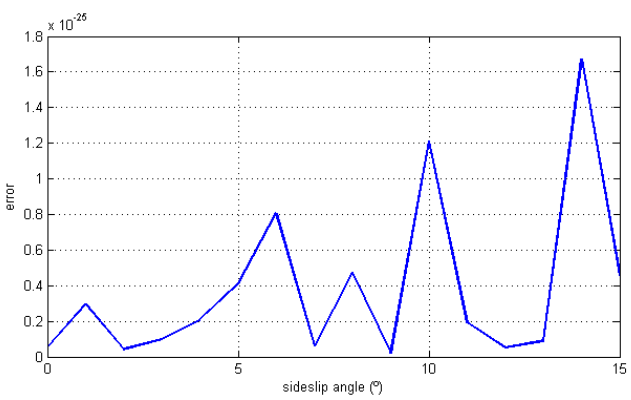

(d) Convergence error

Figure 4.6: Lateral static characteristics in steady sideslip

flight at cruise speed, the resulting trim states and controls are $^{7}$ :

$$
\begin{aligned}
x & =\left[\begin{array}{llllllll}
u & v & w & p & q & r & \phi & \theta
\end{array}\right] \\
& =\left[\begin{array}{llllllll}
29.91 & 0 & 2.35 & 0 & 0 & 0 & 0 & 4.51
\end{array}\right] \\
u & =\left[\begin{array}{llll}
\delta_{a} & \delta_{e} & \delta_{r} & \delta_{t}
\end{array}\right] \\
& =\left[\begin{array}{lllll}
0.151 & -5.25 & 0.015 & 0.841
\end{array}\right]
\end{aligned}
$$

$x$ is not the complete state vector, but the vector of the most relevant $\operatorname{states}^{8}$.

An interesting result is that, despite the fact that a purely longitudinal solution is searched for, the lateral controls are not zero, though they are quite small. This is a consequence of some, but negligible, coupling, due to propeller torque.

It is now necessary to linearise the system around this trim point. Running the linearization algorithm with a tolerance of $0.01 \%$ leads to the following dynamic and control matrices, according to equation (2.33):

\footnotetext{
${ }^{7}$ Angle unit is degree.

${ }^{8}$ The states missing are yaw angle and the one representing engine dynamics.
} 


$A=$\begin{tabular}{ccccccccccc|c}
$u$ & $w$ & $q$ & $\theta$ & $\Omega$ & $v$ & $p$ & $r$ & $\phi$ & $\psi$ \\
\hline-0.0956 & 0.3974 & -2.0713 & -9.7694 & 0.0072 & 0 & -0.0015 & -0.2225 & 0 & 0 & $u$ \\
-0.3636 & -3.6767 & 27.8489 & -0.7692 & 0 & 0 & 0.2249 & 0.0002 & 0 & 0 & $w$ \\
0.0670 & -0.6186 & -5.0577 & 0.0215 & 0 & 0 & -0.0063 & -0.3719 & 0 & 0 & $q$ \\
0 & 0 & 1 & 0 & 0 & 0 & 0 & 0.0141 & 0 & 0 & $\theta$ \\
3.3469 & 0 & 0 & 0 & -0.6241 & 0 & 0 & 0 & 0 & 0 & $\Omega$ \\
0.0001 & 0 & 0 & 0 & 0 & -0.3095 & 2.0207 & -29.5455 & 9.7793 & 0 & $v$ \\
0.0109 & 0.0006 & 0 & 0 & -0.0007 & -0.2060 & -8.0840 & 0.9658 & 0 & 0 & $p$ \\
0.0003 & 0 & 0 & 0 & 0 & 0.1494 & -0.4319 & -0.4041 & 0 & 0 & $r$ \\
0 & 0 & 0 & 0 & 0 & 0 & 1 & 0.0646 & -0.0291 & 0 & $\phi$ \\
0 & 0 & 0 & 0 & 0 & 0 & 0 & 1.0020 & -0.4509 & 0 & $\psi$
\end{tabular}

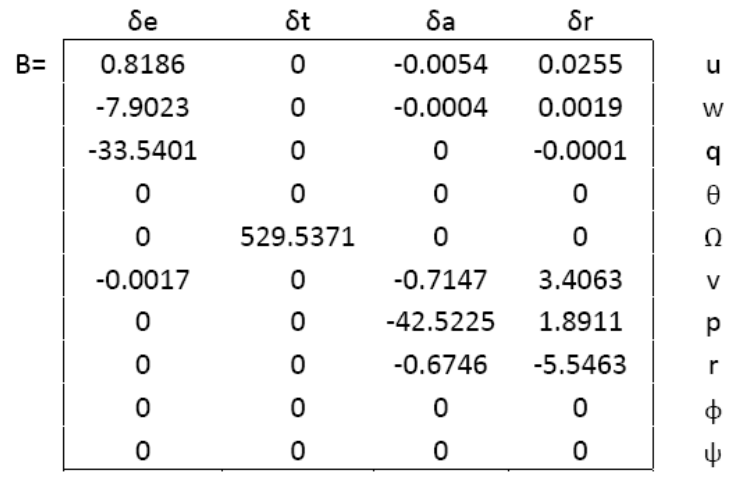

From now on the system described by the above matrices will be referred to as Linearised Model. The disturbances that define the limits of validity of this model model are:

\begin{tabular}{|ccccc|}
\hline$u(\mathrm{~m} / \mathrm{s})$ & $w(\mathrm{~m} / \mathrm{s})$ & $q\left({ }^{\circ} / \mathrm{s}\right)$ & $\theta\left(^{\circ}\right)$ & $\Omega(\mathrm{rad} / \mathrm{s})$ \\
\hline \hline $\mathbf{0 . 0 2 6 3}$ & $\mathbf{0 . 1 3 2 5}$ & 25.78 & $\mathbf{0 . 8 0 8}$ & 0.8485 \\
& & & & \\
\hline
\end{tabular}

\begin{tabular}{|ccccc|}
\hline$v(\mathrm{~m} / \mathrm{s})$ & $p\left({ }^{\circ} / \mathrm{s}\right)$ & $r\left({ }^{\circ} / \mathrm{s}\right)$ & $\phi\left(^{\circ}\right)$ & $\psi\left(^{\circ}\right)$ \\
\hline \hline $\mathbf{0 . 2 2 5}$ & 25.78 & 25.78 & $\mathbf{0 . 8 0 8}$ & 25.78 \\
& & & & \\
\hline
\end{tabular}

\begin{tabular}{|llll|}
\hline$\delta_{e}\left(^{\circ}\right)$ & $\delta_{t}\left(^{\circ}\right)$ & $\delta_{a}\left(^{\circ}\right)$ & $\delta_{r}\left(^{\circ}\right)$ \\
\hline \hline & & & \\
25.78 & 0.0007 & 25.78 & 25.78 \\
& & & \\
\hline
\end{tabular}

Table 4.5: Maximum disturbances for linearisation validity

Disturbances highlighted in bold require particular attention, because they are quite small and are easily exceeded during simulations. 
The relevant eigenvalues/poles of the system are represented and identified on the complex plane, in Figure $4.7^{9}$.

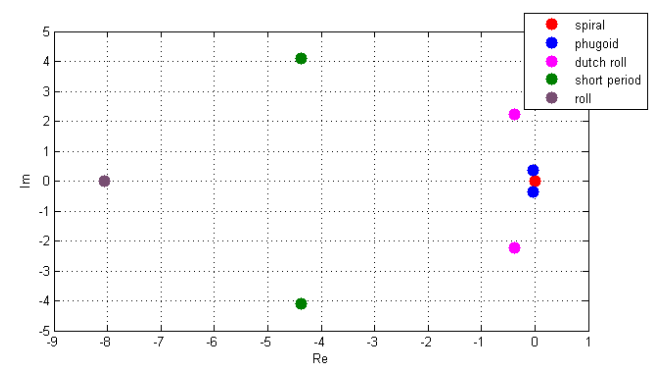

Figure 4.7: Poles of matrix $A$

The exact location, undamped frequency and damping ratio of the different poles are listed on Table 4.6.

\begin{tabular}{|l|ccc|}
\hline Mode & Pole & $\omega_{n}(\mathrm{rad} / \mathrm{s})$ & $\xi$ \\
\hline Short Period & $-4.37 \pm 4.11 i$ & 6.00 & 0.728 \\
Phugoid & $-0.031 \pm 0.347 i$ & 0.348 & 0.089 \\
Roll & -8.05 & 8.05 & 1 \\
Dutch Roll & $-0.390 \pm 2.24 i$ & 2.27 & 0.172 \\
Spiral & 0.0071 & 0.0066 & -1 \\
\hline
\end{tabular}

Table 4.6: Undamped frequency and damping ratio of the different modes

Except for spiral, all poles lie in the left half plane and therefore respective modes are dynamically stable.

The reader is now invited to look back at matrix $A$, but now on Figure 4.8. Its sparseness shows that, for small perturbations around the trim condition, the system can be decoupled: there is little dependence between lateral and longitudinal variables. Actually lateral variables depend less on longitudinal ones than the opposite (see terms circled in blue). This is as expected result, according to page 17 , about aerodynamic model decoupling.

The same can be done to matrix $B$ (see Figure 4.9), where the above conclusions also apply.

Another way to show that decoupling is a good approximation is by analysing the roots of the longitudinal and lateral submatrixes from Figure 4.8 (see Table 4.7).

There is only a slight, but negligible, difference in spiral and phugoid damping, inferior to $7 \%$. Therefore, we can conclude that decoupling is valid.

The linear decoupled equations for straight level flight were presented on Chapter 2 (equations (2.34)

\footnotetext{
${ }^{9}$ Remaining poles are related to engine dynamics $\Omega$ and yaw angle $\psi$.
} 


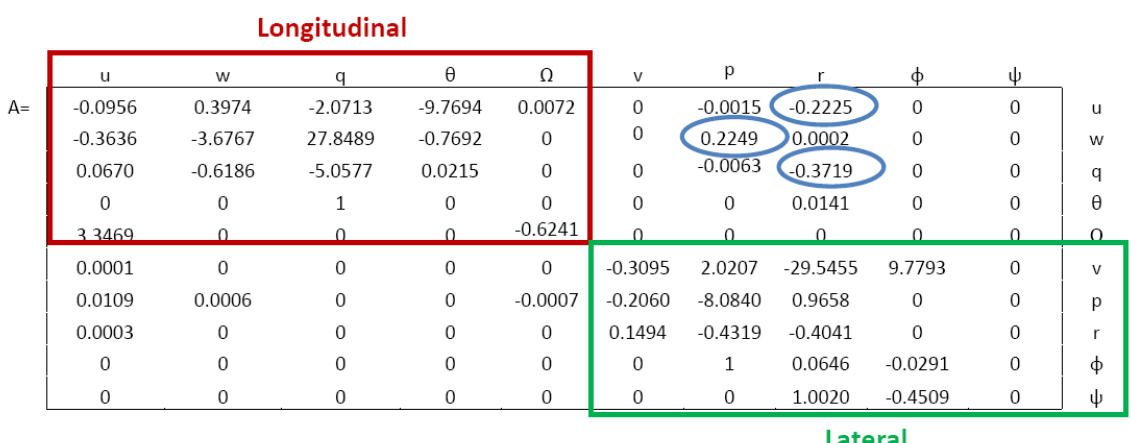

Figure 4.8: Decoupling of matrix $A$

\section{Longitudinal}

$\mathrm{B}=$\begin{tabular}{|cc|cc|c}
\hline$\delta \mathrm{e}$ & $\delta \mathrm{t}$ & $\delta \mathrm{a}$ & \multicolumn{1}{c}{$\delta \mathrm{r}$} \\
\hline 0.8186 & 0 & -0.0054 & 0.0255 & $\mathrm{u}$ \\
-7.9023 & 0 & -0.0004 & 0.0019 & $\mathrm{w}$ \\
-33.5401 & 0 & 0 & -0.0001 & $\mathrm{q}$ \\
0 & 0 & 0 & 0 & $\theta$ \\
0 & 529.5371 & 0 & 0 & $\Omega$ \\
\hline-0.0017 & 0 & -0.7147 & 3.4063 & $\mathrm{v}$ \\
0 & 0 & -42.5225 & 1.8911 & $\mathrm{p}$ \\
0 & 0 & -0.6746 & -5.5463 & $\mathrm{r}$ \\
0 & 0 & 0 & 0 & $\phi$ \\
0 & 0 & 0 & 0 & $\downarrow$ \\
\hline
\end{tabular}

Figure 4.9: Decoupling of matrix $B$

and (2.35)). Because they give physical insight to the different entries of the dynamic matrix and are easier to obtain, it is important to verify if they can be used for further analysis.

The resultant systems from equations (2.34) and (2.35) will be referred to as Longitudinal Linear Model and Lateral Linear Model from now on, respectively, or Linear Decoupled Model, if the two are considered simultaneously.

\section{Longitudinal}

Based on system 2.34, the Longitudinal Linear Model dynamic matrix is given by:

$$
A=\left[\begin{array}{cccc}
-0.1130 & 0.1135 & -2.3580 & -9.7697 \\
-0.6513 & -3.660 & 27.9597 & -0.7695 \\
0.0272 & -0.5720 & -5.4482 & 0.0321 \\
0 & 0 & 1 & 0
\end{array}\right]
$$

This matrix does not account for engine dynamics, expressed by variable $\Omega$, which is the same as considering that the engine responds automatically to the throttle command $\delta_{t}$. 


\begin{tabular}{|cc|}
\hline \multicolumn{2}{|c|}{ Longitudinal Submatrix } \\
\hline Short Period & Phugoid \\
\hline \hline & $\omega_{n}=0.348$ \\
$\omega_{n}=6.00$ & $\xi=0.088$ \\
\hline
\end{tabular}

\begin{tabular}{|ccc|}
\hline \multicolumn{3}{|c|}{ Lateral Submatrix } \\
\hline Roll & Dutch Roll & Spiral \\
\hline \hline & $\omega_{n}=2.27$ & $\omega_{n}=0.0066$ \\
$\omega_{n}=8.05$ & $\xi=0.172$ & $\xi=-1$ \\
\hline
\end{tabular}

Table 4.7: Characteristics of dynamic modes as predicted by the linearised decoupled model

Poles of the above matrix have the characteristics listed on Table 4.8.

\begin{tabular}{|cc|}
\hline \multicolumn{2}{|c|}{ Longitudinal Linear Model } \\
\hline Short Period & Phugoid \\
\hline$\omega_{n}=5.99$ & $\omega_{n}=0.357$ \\
$\xi=0.760$ & $\xi=0.158$ \\
\hline
\end{tabular}

Table 4.8: Characteristics of longitudinal linear model poles

Results are quite similar to the ones from Table 4.7, except for phugoid damping ratio (differences in other parameters are not significative). This discrepancy in phugoid damping ratio results from not considering the engine dynamics, as inspection of poles of longitudinal submatrix $A$, without the last column and line, reveals (see Table 4.9).

The main effect is on phugoid damping. This is an expected result, since the longitudinal speed variable $u$, which reflects that the phugoid mode, is very sensitive to engine dynamics. In fact, by looking at the complete matrix A (see Figure 4.8), or even at the longitudinal submatrix, it is visible that only the longitudinal variable $u$ is affected by the engine dynamics, expressed by state variable $\Omega$, and why phugoid damping ratio is smaller: when, due to phugoid mode, $u$ increases(decreases), $\Omega$ increases (decreases) leading to an increase(decrease) in $u$ (positive $A(5,1)$ ). Phugoid is stable (positive damping) because the damping effect compensates for this tendency.

Usually, a constant break horse power engine like the one equipping ANTEX, tends to improve phugoid damping ratio (see Cook [4]), but, depending on its operating point, it might induce an 


\begin{tabular}{|cc|}
\hline \multicolumn{2}{|c|}{ Longitudinal Submatrix - without engine dynamics } \\
\hline \hline Short Period & Phugoid \\
\hline & $\omega_{n}=0.356$ \\
$\omega_{n}=6.00$ & $\xi=0.132$ \\
$\xi=0.728$ & \\
\hline
\end{tabular}

Table 4.9: Characteristics of longitudinal submatrix poles, without engine dynamics

adversa effect.

\section{Lateral}

Based on equation (2.35), the Lateral Linear Model dynamic matrix is given by:

$$
A=\left[\begin{array}{cccc}
-0.2817 & 2.156 & -29.6639 & 9.7697 \\
-0.2062 & -8.0730 & 0.5521 & 0 \\
0.1496 & -0.2582 & -0.4251 & 0 \\
0 & 1 & 0.0788 & 0
\end{array}\right]
$$

This matrix does not include the lateral variable $\psi$, which, as equations 2.13 show, hasn't any effect on aircraft dynamics.

Poles of the above matrix have the characteristics listed on Table 4.10.It can be verified that results are pretty similar to the ones from Table 4.7, except for spiral, whose unstability has got worse, that is to say, the spiral spole as moved further away from the imaginary axis (to the right).

\begin{tabular}{|ccc|}
\hline \multicolumn{3}{|c|}{ Lateral Linear Model } \\
\hline Roll & Dutch Roll & Spiral \\
\hline \hline$\omega_{n}=8.06$ & $\omega_{n}=2.24$ & $\omega_{n}=0.0227$ \\
$\xi=1$ & $\xi=0.166$ & $\xi=-1$ \\
\hline
\end{tabular}

Table 4.10: Characteristics of lateral linear model poles

Comparing previous dynamic matrix with lateral submatrix from Figure 4.8, there are significant differences in some of their entries, but some tests (not reproduced here) have revealed that entry $A(2,2)$ might be an important source for this difference. This term represents the effect of $\phi$, the variable reflecting spiral effects, in $\dot{\phi}$, which, according to system 2.35 is zero. But it should be reminded that, for the Linearised Model, the maximum perturbation in $\phi$ is very small (see Table 4.5), less than $1^{\circ}$, which leads to some uncertainty on spiral results. 
To understand the real characteristics of spiral mode, it is important to look at the nonlinear system. Results from nonlinear simulations are presented later in this section.

Because some responses to inputs have to be analysed, control matrixes should also be compared. For the Lateral Linear Model we have:

$$
B=\left[\begin{array}{cc}
-0.7164 & 3.4144 \\
-42.6233 & 1.8955 \\
-0.6762 & -5.5594 \\
0 & 0
\end{array}\right]
$$

The similarity between this and the lateral control submatrix from Figure 4.8 is evident.

Lateral flying qualities not only include undamped frequency and damping ratio of the different modes, but other parameters for spiral and roll modes, whose determination follows. For their study, both the Lateral Linear Model and Linearised Model are used. The objective is, again, to compare the results and see if the decoupled model, which is simpler, can be used for further studies.

\section{Spiral}

Spiral is unstable and, therefore, the time to double amplitude must be determined. According to Appendix C, equation C.8, it is related to the spiral pole, through the following expression:

$$
T_{2}=\frac{0.6931}{n}
$$

where $n$ is the real part of spiral pole.

To prove the validity of this expression we also measure $T_{2}$ directly from $\phi$ response to a rudder input. The input is as suggested in Table 3.2: step and removal after $\phi=20^{\circ} . T_{2}$ is the time it takes for roll angle to reach $\phi=40^{\circ}$.

One smal note should be made on $\phi$ response: depending on rudder deflection, $T_{2}$ might vary a little, so the mean value for three different deflections $\left(5^{\circ}, 10^{\circ}\right.$ and $\left.12^{\circ}\right)$ is presented. This might be related to initial oscillations, probably resulting from dutch roll, which, because of its short period nature, affects the initial response (see Figure 4.10).

Table 4.11 presents the results obtained using the different methods.

\begin{tabular}{|cc|cc|}
\hline \multicolumn{2}{|c|}{ Lateral Linear Model } & \multicolumn{2}{c|}{ Linearised Model } \\
\hline Equation C.8 & $\phi$ Response & Equation C.8 & $\phi$ Response \\
\hline \hline 30.5 & 31.2 & 98.3 & 101.2 \\
& & & \\
\hline
\end{tabular}

Table 4.11: Time to double for spiral mode

As predicted, equation (C.8) gives good results and can be used for $T_{2}$ prediction. The difference in values from the two models has been addressed to before. 


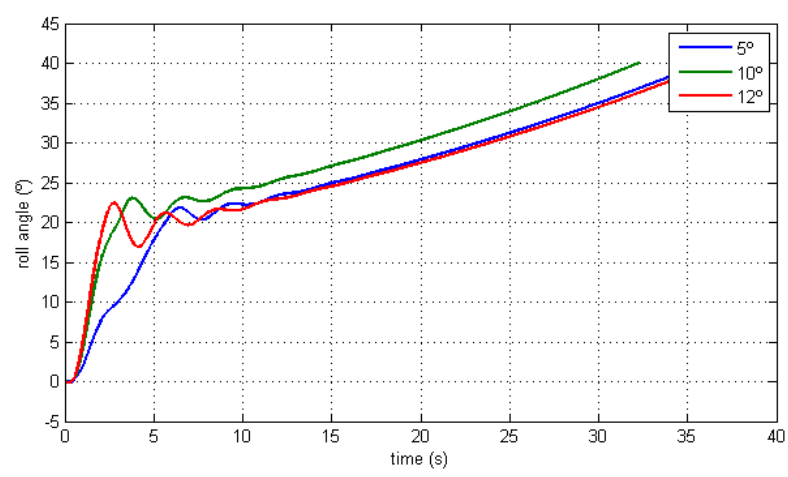

Figure 4.10: Spiral excitation with three different rudder pulses

\section{Roll Performance}

According to Appendix C, the time constant of a first order mode is given by the relation

$$
\tau=\frac{1}{a}
$$

where $a$ is the absolute value of the eigenvalue.

Determination of time to reach $\phi=60^{\circ}\left(\left.t\right|_{\phi=60^{\circ}}\right)$ is made through analysis of $\phi$ response to a step input on aileron, with magnitude equal to its maximum deflection $\left(15^{\circ}\right)$.

Results are presented on Table 4.12 .

\begin{tabular}{|cc|cc|}
\hline \multicolumn{2}{|c|}{ Lateral Linear Model } & \multicolumn{2}{|c|}{ Linearised Model } \\
\hline$\tau(s)$ & $\left.t\right|_{\phi=60^{\circ}}(s)$ & $\tau(s)$ & $\left.t\right|_{\phi=60^{\circ}}(s)$ \\
\hline \hline & & & \\
0.124 & 0.91 & 0.124 & 0.93 \\
& & & \\
\hline
\end{tabular}

Table 4.12: Roll performance characteristics

\subsubsection{Nonlinear Response}

The nonlinear system is now used for flying qualities prediction, by performing simulations as described on Table 3.2. In order to minimise possible errors, we test three different inputs for each parameter. Then, FQ parameters are measured according to Appendix C. Only the final results are presented.

Limits for linearization validity (see Table 4.5), are purposely exceeded in some cases to check if differences are significant. On the other hand, some are so small (the ones highlighted in bold) that it is difficult to avoid it.

Comparison with previous results is left for next section. 


\section{Simulation 1. Short Period}

Input: Elevator Doublet

\begin{tabular}{|c|c|c|}
\hline Input & Amplitude $\left(^{\circ}\right)$ & Total Duration $(s)$ \\
\hline \hline & & \\
1 & 5 & 0.1 \\
2 & 10 & 0.1 \\
3 & 3 & 0.2 \\
\hline
\end{tabular}

Variable of Interest: angle of attack $\alpha$

\section{Response:}

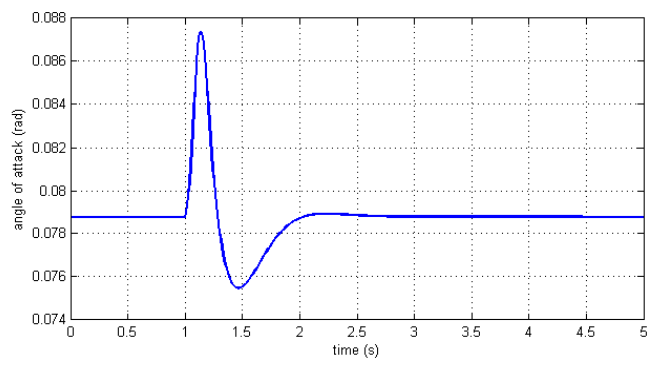

Angle of attack response to elevator doublet

\section{Results:}

\begin{tabular}{|c|cc|cc|}
\hline Input & $\xi$ & Mean $\xi$ & $\omega_{n}(\mathrm{rad} / \mathrm{s})$ & Mean $\omega_{n}(\mathrm{rad} / \mathrm{s})$ \\
\hline \hline & & & & \\
1 & 0.56 & & 6.92 & \\
2 & 0.59 & 0.57 & 7.09 & 6.99 \\
3 & 0.56 & & 0.697 & \\
\hline
\end{tabular}

\section{Comments:}

In spite of the efforts made to avoid it, there is a little coupling with phugoid mode, specially close to the second peak. This difficults correct measurement of peak values.

\section{Simulation 2. Phugoid}

Input:Elevator Pulse 


\begin{tabular}{|c|c|c|}
\hline Input & Amplitude $\left(^{\circ}\right)$ & Total Duration $(s)$ \\
\hline \hline & & \\
1 & 5 & 1 \\
2 & 3 & 0.2 \\
3 & 10 & 0.8 \\
\hline
\end{tabular}

Variable of Interest: longitudinal speed $u$ Response:

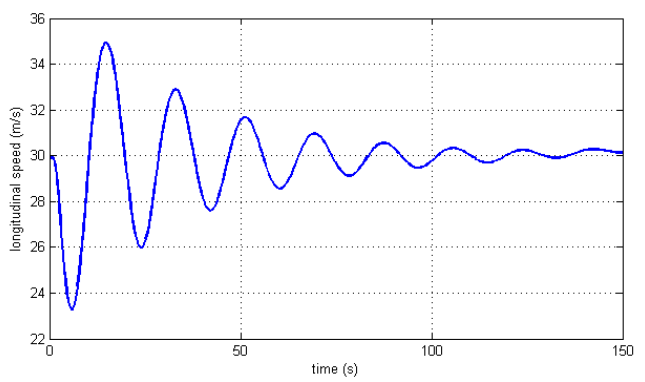

Longitudinal speed response to elevator pulse

Results:

\begin{tabular}{|c|cc|cc|}
\hline Input & $\xi$ & Mean $\xi$ & $\omega_{n}(\mathrm{rad} / \mathrm{s})$ & Mean $\omega_{n}(\mathrm{rad} / \mathrm{s})$ \\
\hline \hline & & & & \\
1 & 0.0835 & & 0.347 & \\
2 & 0.0885 & 0.0852 & 0.347 & 0.347 \\
3 & 0.0872 & & 0.347 & \\
\hline
\end{tabular}

\section{Comments:}

Calculations are based on first, third and sixth peaks.

\section{Simulation 3. Roll Performance}

Input: aileron step

\begin{tabular}{|c|c|}
\hline Input & Amplitude $\left(^{\circ}\right)$ \\
\hline \hline & \\
1 & 5 \\
2 & 10 \\
3 & 15 \\
\hline
\end{tabular}

Variable of interest: roll rate $p$ and roll angle $\phi$ 
Response:

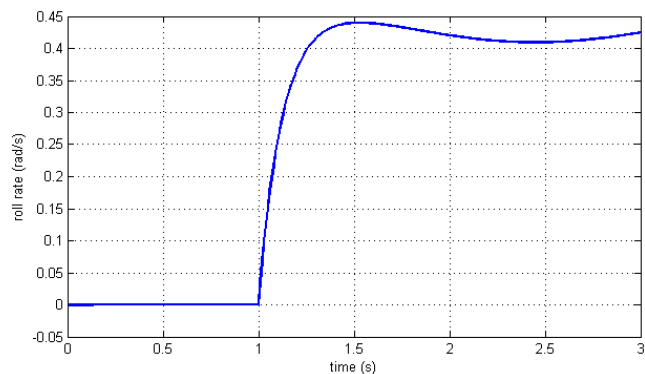

Roll rate response to aileron step

\section{Results:}

\begin{tabular}{|c|c|c|c|}
\hline Input & Time Constant $(s)$ & Mean Time Constant $(s)$ & Time to reach $\left.\phi=60^{\circ}\right)$ \\
\hline \hline & & & \\
1 & 0.109 & 0.112 & \\
2 & 0.113 & & 0.899 \\
3 & 0.114 & & \\
\hline
\end{tabular}

\section{Comments:}

There is some coupling with dutch roll mode, particularly after the quick initial increase in $p$, which difficults measurement of its apparent steady-state value, used for time constant determination.

\section{Simulation 4. Spiral Stability}

Input: Rudder Pulse Amplitude and duration were chosen so that $\phi$ would be approximately $20^{\circ}$ in the end of the pulse.

\begin{tabular}{|c|c|c|}
\hline Input & Amplitude $\left({ }^{\circ}\right.$ & Total Duration $(s)$ \\
\hline \hline & & \\
1 & 5 & 6 \\
2 & 10 & 3 \\
3 & 3 & 9 \\
\hline
\end{tabular}

Variables of interest: roll angle $\phi$ 


\section{Response:}

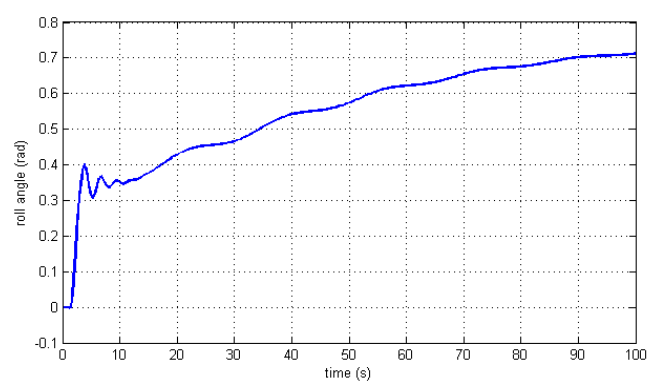

Roll angle response to rudder pulse

\section{Results:}

\begin{tabular}{|c|c|c|}
\hline Input & Time to Double $(s)$ & Mean Time to Double \\
\hline \hline & & \\
1 & 69.3 & \\
2 & 68.2 & 69.2 \\
3 & 70.1 & \\
\hline
\end{tabular}

\section{Comments:}

After the pulse is removed, some oscillations are visible, as had already happened with linear results (see Figure4.10).

\section{Simulation 5. Dutch Roll}

Input: Rudder Doublet Though a period similar to the one of the mode is suggested for flight tests, we tested others as well. Though a period similar to the one of the mode is suggested for flight tests, we tested others as well.

\begin{tabular}{|c|c|c|}
\hline Input & Amplitude $\left(^{\circ}\right.$ & Total Duration $(s)$ \\
\hline \hline & & \\
1 & 5 & 2 \\
2 & 10 & 0.5 \\
3 & 3 & 1 \\
\hline
\end{tabular}

Variables of interest: roll angle $\phi$ and sideslip angle $\beta$ 


\section{Response:}

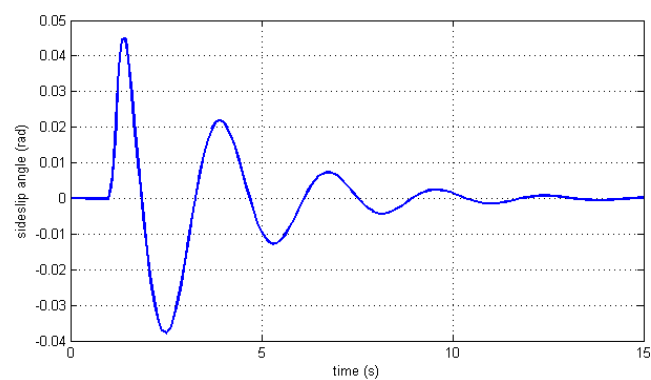

Sideslip angle response to rudder doublet

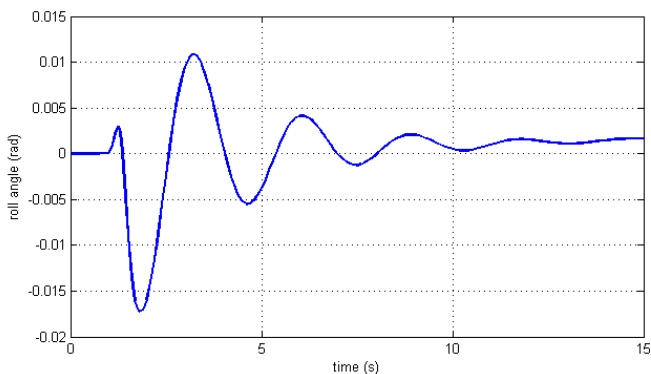

Roll angle response to rudder doublet

\section{Results:}

Undamped frequency and damping ratio were determined based on sideslip response. Because of $\phi$ divergence due to spiral mode, calculations based on its response are more difficult and error prone.

\begin{tabular}{|c|cc|cc|}
\hline Input & $\xi$ & Mean $\xi$ & $\omega_{n}(\mathrm{rad} / \mathrm{s})$ & Mean $\omega_{n}(\mathrm{rad} / \mathrm{s})$ \\
\hline \hline & & & & \\
1 & 0.173 & & 2.24 & \\
2 & 0.174 & 0.173 & 2.26 & 2.25 \\
3 & 0.174 & & 2.24 & \\
\hline
\end{tabular}

\section{Comments:}

Calculations are based on first three peaks.

In all of the studied parameters, results for the three different input are quite similar, even when leading to perturbations above the linear limits presented on Table 4.5. This fact will be used in next section for results comparison. 


\subsubsection{Dynamic Flying Qualities Collected}

Previous results have been presented in a quite sparse manner, due to their detailed analysis, and so this section was created to summarise them in one single place. Two situations are interesting to look closely:

- Comparison between Linearised Model and Nonlinear Model results

- Comparison between Linear Decoupled Model and Linearised Model results

Tables 4.13 and 4.14 put the different results side-by-side and show the error between them, calculated through the following expressions:

\section{Linearised vs Nonlinear}

$$
e=\frac{\mid \text { value }_{\text {Lin }}-\text { value }_{\text {Nonlin }} \mid}{\mid \text { value }_{\text {Nonlin }} \mid} \times 100
$$

\section{Linear vs Linearised}

$$
e=\frac{\mid \text { value }_{\text {Linear }}-\text { value }_{\text {Linearised }} \mid}{\mid \text { value }_{\text {Linearised }} \mid} \times 100
$$

\begin{tabular}{|c|cc|c|}
\hline FQ & Linearised Model & Nonlinear Model & Error (\%) \\
\hline \hline & & & \\
$\omega_{n_{p h}}$ & 0.348 & 0.347 & 0.29 \\
$\xi_{p h}$ & 0.088 & 0.0852 & 3.53 \\
$\omega_{n_{s p}}$ & 6.00 & 6.99 & 14.16 \\
$\xi_{s p}$ & 0.728 & 0.57 & 27.72 \\
$\tau_{\text {roll }}$ & 0.124 & 0.112 & 10.71 \\
$\left.t\right|_{\phi=60^{\circ}}$ & 0.93 & 0.90 & 3.33 \\
$T_{2_{s p i r a l}}$ & 98.3 & 69.2 & 42.01 \\
$\omega_{n_{d r}}$ & 2.27 & 2.25 & 0.89 \\
$\xi_{d r}$ & 0.172 & 0.173 & 0.58 \\
\hline
\end{tabular}

Table 4.13: Comparison between linearised model and nonlinear model results

Table 4.13 shows that most results are very similar, with errors inferior to $4 \%$, even with nonlinear simulations overcoming validity limits for the linearised model.

However, significant discrepancies are found on short period parameters, roll time constant and spiral time to double amplitude. The first two can be justified by the measurement errors identified in the nonlinear section. As to the spiral mode, the linearised model overestimates stability (higher $T_{2}$ ). In fact, its validity limits as set by $\phi$, the spiral variable par excellence, are prohibitevely small and 
might be the source of the error. If the linearised model predicted a worst scenario in terms of spiral stability, this big difference would not require so much attention, but as this is not the case, further analysis must not disregard this result.

Looking at Table 4.14, the same problem with spiral mode is found, though this time the linear model presents a worst scenario, even worse than that of the nonlinear model. There is also a considerable difference in phugoid damping ratio. Both problems have been identified and explained before (see pages 57 and 56).

We can conclude that the Linear Decoupled Model gives quite accurate results and, therefore, can be used for parametric studies purposes.However phugoid damping ratio and spiral stability require special attention: conclusions to draw shall be validated through the linearised and nonlinear models, respectively.

\begin{tabular}{|c|cc|c|}
\hline FQ & Linear Decoupled Model & Linearised Model & Error (\%) \\
\hline \hline & & & \\
$\omega_{n_{p h}}$ & 0.356 & 0.348 & 2.30 \\
$\xi_{p h}$ & 0.158 & 0.088 & 79.55 \\
$\omega_{n_{s p}}$ & 5.99 & 6.00 & 0.17 \\
$\xi_{s p}$ & 0.76 & 0.728 & 4.40 \\
$\tau_{\text {roll }}$ & 0.124 & 0.124 & 0 \\
$t \mid{ }_{-}=60^{\circ}$ & 0.91 & 0.93 & 3.33 \\
$T_{2_{s p i r a l}}$ & 30.5 & 98.3 & 68.97 \\
$\omega_{n_{d r}}$ & 2.24 & 2.27 & 1.32 \\
$\xi_{d r}$ & 0.166 & 0.172 & 3.49 \\
\hline
\end{tabular}

Table 4.14: Comparison between between linear decoupled model and linearised model results

\subsection{Qualitative Analysis of Flying Qualities}

The different fying qualities parameters have been measured. But just quantification is not enough to evaluate flying qualities: it is important to analyze results qualitatively. Because criteria for UAVs is still under development, the available option is to use piloted guidelines. Actually, this has been the followed approach for UAV development up to date.

Evaluation depends on type of aircraft, usually know in flying qualities area as class, and flight phase. In Section 3.3, we mentioned this type of classification and, according to it, ANTEX falls under class I and category B. A summary of the most relevant criteria for class I, category B, can be found on Table 4.15 .

According to piloted aircraft criteria, ANTEX flying qualities are level 1, with its current characteristics well above the minimum limits. This is valid for both linear and nonlinear results. 


\begin{tabular}{|c|c|c|c|}
\hline Dyn. Mode & Level 1 & Level 2 & Level 3 \\
\hline Phugoid & $\xi_{p h} \geq 0.04$ & $\xi_{p h} \geq 0$ & $T_{2_{p h}} \geq 55 \mathrm{~s}$ \\
\hline Short Period & $2 \geq \xi_{s p} \geq 0.3$ & $2 \geq \xi_{s p} \geq 0.2$ & $\xi_{s p} \geq 0.15$ \\
\hline Roll & $\begin{aligned} \tau & \leq 1.4 \\
\left.t\right|_{\phi=60^{\circ}} & \leq 1.7\end{aligned}$ & $\begin{aligned} \tau & \leq 3 \\
\left.t\right|_{\phi=60^{\circ}} & \leq 2.5\end{aligned}$ & $\begin{aligned} \tau & \leq 10 \\
\left.t\right|_{\phi=60^{\circ}} & \leq 3.4\end{aligned}$ \\
\hline Spiral & $T_{2_{\text {spiral }}}>20 \mathrm{~s}$ & $T_{2_{\text {spiral }}}>8 \mathrm{~s}$ & $T_{2_{\text {spiral }}}>4 \mathrm{~s}$ \\
\hline Dutch Roll & $\begin{aligned} \xi_{d r} & \geq 0.08 \\
\xi_{d r} \omega_{n_{d r}} & \geq 0.15 \mathrm{rad} / \mathrm{s} \\
\omega_{n_{d r}} & \geq 0.4 \mathrm{rad} / \mathrm{s}\end{aligned}$ & $\begin{aligned} \xi_{d r} & \geq 0.02 \\
\xi_{d r} \omega_{n_{d r}} & \geq 0.05 \mathrm{rad} / \mathrm{s} \\
\omega_{n_{d r}} & \geq 0.4 \mathrm{rad} / \mathrm{s}\end{aligned}$ & $\begin{array}{c}\xi_{d r} \geq 0 \\
- \\
\omega_{n_{d r}} \geq 0.4 \mathrm{rad} / \mathrm{s}\end{array}$ \\
\hline
\end{tabular}

Table 4.15: Flying qualities requirements for Class I, category B

Having good piloted-based flying qualities is not necessarily good or bad for a UAV. But this is the available information, and analysis of what can be improved (Chapter 6) will be based on the existing requirements.

Static characteristics also comply with requirements, but it is desirable that elevator solicitation at low speed is reduced.

\subsection{Effect of Spinning Rotors}

Previous studies did not account for the existence of spinning rotors, but it is important to investigate the consequences of doing such simplification.

Equation (2.11) shows that this effect is only present when angular velocity is not zero. Therefore, we can conclude that static characteristics determined from flight conditions such as straight level flight and steady sideslip are not affected. All the remaining parameters might vary.

The convergence error for new $\delta_{e}$ vs $n$ and $\alpha$ vs $n$ curves is around $10^{-2}$ which is higher than the convergence criterion from Section 2.3. For that reason, and although the results obtained approach the ones not considering spinning rotors effect, we opted not to presented them.

But dynamic characteristics are the most interesting to analyse. Table 4.16 present the new results, which were obtained through the linearised model, except for spiral - considering previous conclusions, we used the nonlinear model for $T_{2}$ prediction.

New results are pretty similar to the previous ones, except for spiral, where error is significant (over (20\%). Still, the new $T_{2}$ value is quite large, over twice the limit for level $1 \mathrm{FQ}$ (see Table 4.15), and 


\begin{tabular}{|c|cc|c|}
\hline Flying Qualities & $\begin{array}{c}\text { with spinning } \\
\text { rotors effect }\end{array}$ & $\begin{array}{c}\text { without spinning } \\
\text { rotors effect }\end{array}$ & Error (\%) \\
\hline \hline & & & \\
$\omega_{n_{p h}}$ & 0.348 & 0.348 & 0 \\
$\xi_{p h}$ & 0.094 & 0.088 & 6.82 \\
$\omega_{n_{s p}}$ & 6.00 & 6.00 & 0 \\
$\xi_{\text {sp }}$ & 0.723 & 0.728 & 0.69 \\
$\tau_{\text {roll }}$ & 0.123 & 0.124 & 0.81 \\
$t \mid \phi=60^{\circ}$ & 0.93 & 0.93 & 0 \\
$T_{2_{\text {spiral }}}$ & 52.7 & 69.2 & 23.8 \\
$\omega_{n_{d r}}$ & 2.28 & 2.27 & 0.44 \\
$\xi_{d r}$ & 0.184 & 0.172 & 6.98 \\
\hline
\end{tabular}

Table 4.16: Comparison between results with and without spinning rotors effect so we can safely neglect spinning rotors effect. 


\section{Chapter 5}

\section{Parametric Studies}

The main goal of this work is to determine the influence of some aircraft design parameters, related to size and position of the control surfaces and stabilizers, on a set of flying qualities.

Flying qualites are determined from the adynamic model, whose dependence on design is expressed mainly by the aerodynamic model and mass properties. The way these effects come out depends on the aircraft configuration and, therefore, it is difficult to generalise any conclusions that might be drawn. Nevertheless, if an approach exists that allows generalization to some point, it should not be disregarded, since it might bring some advantages for the task of extrapolating results to different aircraft.

Despite the differences in flight mechanics inherent to a particular configuration, there is a general form for an aircraf 6-DOF dynamic model that can be used for simulation of different aircraft types. The approach we follow to perform parametric studies takes advantage of this fact by dividing the analysis in two parts: a more general one regarding only the model structure and therefore not looking to a specific configuration, and another one which studies how the model parameters depend on a specific aircraft design.

\section{$5.1 \quad$ Strategy}

We divide the parametric study in two parts, both based on sensitivity analysis. The first one analyzes the sensitivity of flying qualities to a change in model parameters, particularly aerodynamic characteristics and mass properties, and the second investigates the dependence of these model parameters on a set of design variables. Then, putting the results together makes it is possible to understand the influence of design parameters on flying qualities.

This could be done directly, without the intermidiate step based on model parameters. However, 
in spite of being more straightforward, this method has at least two disadvantages:

- Difficulty in extrapolating results to other configurations

- Loss of physical insight into which factors are really causing flying qualities to change

Splitting the analysis in two avoids that, but is also synonym of increasing work. Additionally, because an aerodynamic model usually requires over 20 derivatives, it might become even more time consuming. That is why sensitivity analysis are so important: they allow the identification of the relevant contributions. In fact,

- Not all model parameters are sensitive to the selected design parameters.

- For the set of flying qualities under study, some model parameters are more important than others.

Based on theses facts, we can reduce the set of model parameters to consider.

According to conclusions from Chapter 4, we can use the Linear Decoupled Model from equations 2.34 and 2.35 to study flying qualities dependence on the several model parameters. This greatly simplifyies the process. Analysis of design parameters effects on aerodynamic derivatives is based on empiric expressions from Appendix B, since we have seen in last chapter that they reasonably predict CFD results; for mass properties determination, we model the aircraft with known regular volumes.

\subsection{Choice of Design Parameters}

The chosen design parameters are represented in Figure 5.1. This choice was based on FOI interest in specifically studying control surfaces and stabilizers.

\subsection{Sensitivity Analysis}

\subsubsection{Overview}

Let $y$ be a function of several variables. A sensitivity analysis determines how a change in any of those variables affects $y$. The objective is to find those which have more influence on $y$, the major drivers. It is important to say that sensitivity analysis work better for well-behaved functions, that is to say, continuous and differenciable in their domain.

Besides the identification of the relevant variables, sensitivity analysis allows them to be sort in terms of relative importance, which helps to decide on which parameters one should invest more resources. On the other hand, it is possible to check how the uncertainties present in both the aerodynamic and mass properties models might affect the results. This adds even more value to the analysis, because, despite the powerful tools available today, simulations or real testing are almost certainly contaminated with noise. 

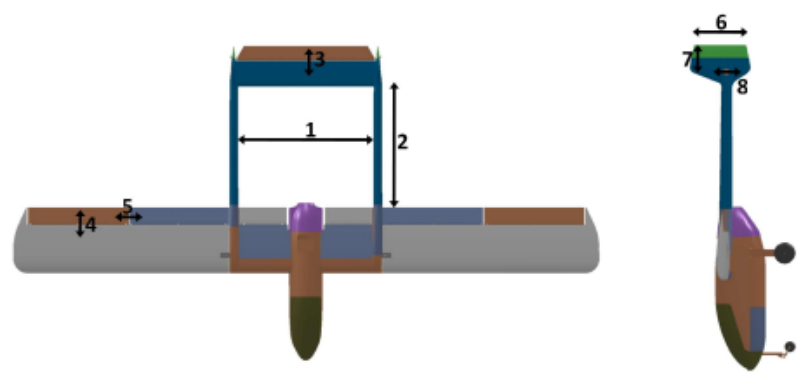

Legend:

1. Distance between tail booms

2. Tail boom length

3. Elevator hinge line position

4. Aileron chord

5. Aileron length

6. Rudder/Vertical Stabilizer length

7. Rudder hinge line position

8. Elevator/Horizontal Stabilizer vertical position

Figure 5.1: Design Parameters

\subsubsection{Flying Qualities Sensitivity to Model Parameters}

The idea of studying the dependence of some flying qualities on model parameters is not new. For example, Roskam [17] presents plots of dynamic stability characteristics such as undamped frequency, damping ratio and time constant of the different modes, as a function of aerodynamic derivatives and mass properties allowed to vary over a certain range, for a business jet in cruise. The way he organises this information is as follows:

- One plot for each derivative/mass property

- One plot for longitudinal modes and other to lateral modes

This might difficult a correct interpretation of results in two ways:

- The same scale is used for the different modes parameters, which, as already seen on Chapter 4, do not have the same order of magnitude. As a result, their variation might be misinterpreted.

- Effects of the different derivatives/mass properties are not plotted against each other, which might difficult their line-up in terms of importance.

But Roskam also provides a writen interpretation of the results.

On the other hand, other authors (see e.g. [19]) present the same effects through longitudinal/lateral root locus plots, again one for each model parameter allowed to vary. 
It is also frequent to find similar information, in a somewhat different, and apparently not so obvious, fashion, which is related to reduced-order models (see e.g. [7]). These are 2-DOF or even 1-DOF models based on the physical interpretation of the different longitudinal and lateral modes. Decoupling of the linearised 6-DOF model already represented great simplification, and this order reduction takes it further. Besides, it also filters the model parameters that are relevant for each mode.

Comparing with the full longitudinal or lateral linear model results, approximations are quite reasonable, except for spiral, dutch roll damping ratio and phugoid, where errors might be significant. We didn't use these reduced-order models in previous analysis to avoid more simplification, which is a synonim of less accuracy, and because 4-DOF models (like the longitudinal and lateral) are easy to deal with using a software like MATLAB.

In spite of the fact that some results already exist, we decided to make a new sensitivity analysis for four main reasons:

- Additional model parameters and flying qualities are to analysed

- It is important to plot the effects of the different model parameters against each other, for the reason already mentioned

- To avoid misinterpretation due to scaling differences, there is the need to study each flying quality parameter independently

- Because a specific aircraft is studied in this project, it would be interesting to check if results are similar to the existing ones

For this study, and because it is intended to provide results as general as possible, we allow wide variations of model parameters, sometimes up to \pm 10 times their baseline value, even if it might lack physical meaning. However, some parameters produce quite large effects and we carefully choose the range of variation so the function is well-behaved.

Flying qualities dependence on model parameters could also be studied analitically, through respective functional relations, but these are quite complex, not only due to the fact that we have a $4 t h$ order system, but also because of the great amoutn of parameters to analyse. The approach followed reflects the same results, but in a simpler and easier to understand fashion (graphically), giving more physical insight. 


\subsubsection{Longitudinal}

\section{Dynamic Characteristics}

Longitudinal dynamic characteristics under study only depend on the dynamic matrix. We test those parameters over which this matrix is built ${ }^{1}$. Figures 5.2 and 5.3 illustrate longitudinal dynamic modes sensitivity to model parameters, whose domain of variation is represented in the $x$ axis by factors that multiply the baseline value, from -10 to 10 .

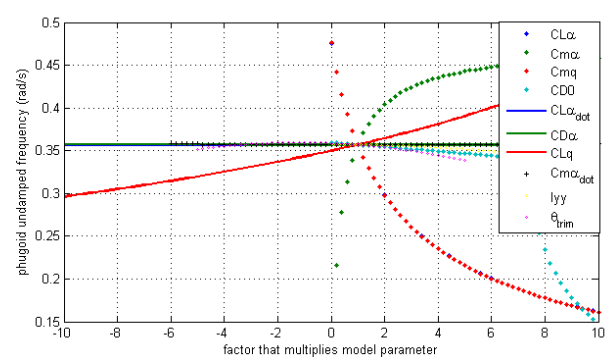

(a) Phugoid undamped frequency

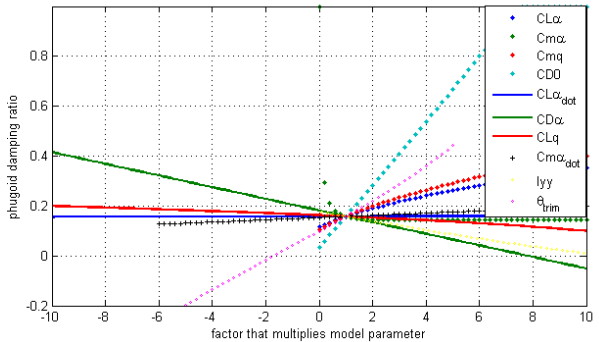

(b) Phugoid damping ratio

Figure 5.2: Phugoid sensitivity to model parameters

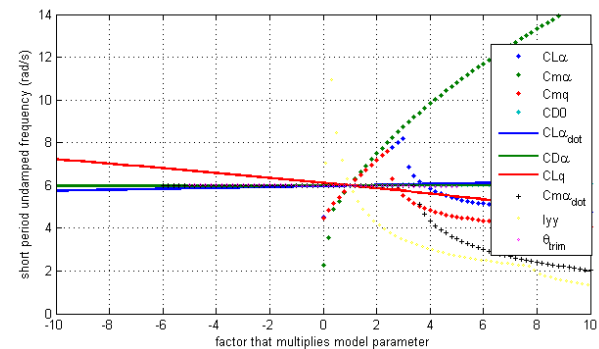

(a) Short period undamped frequency

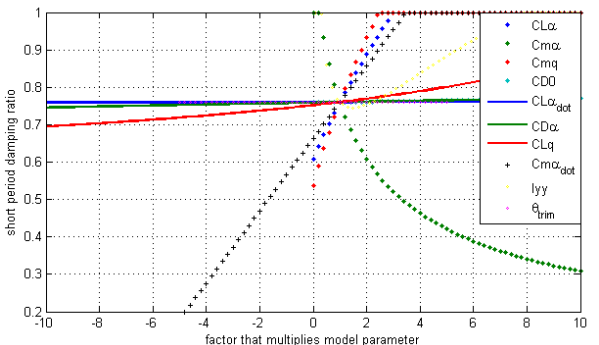

(b) Short period damping ratio

Figure 5.3: Short period sensitivity to model parameters

\section{Static Characteristics}

It is not worth repeating the analysis for static characteristics, as equations 3.1 to 3.7 already show which elements shall be taken into consideration. However, inspection of effects due to $C_{L_{q}}$ leads to an interesting result: it has little influence on elevator and angle of attack trim angles in steady pull-ups/push-overs (see Figures 5.4).

\footnotetext{
${ }^{1}$ For the flight phase we study, straight level flight, $\alpha_{\text {trim }}=\theta_{\text {trim }}$. Therefore, we only present $\theta_{\text {trim }}$ effects, both for longitudinal and lateral cases.
} 


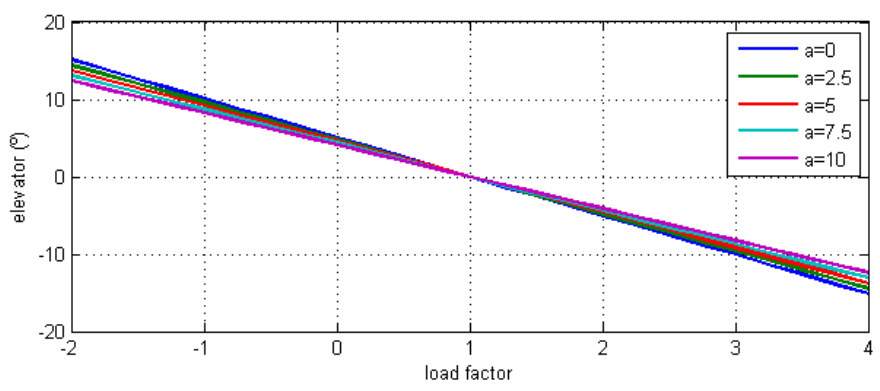

(a) Elevator position per $n$

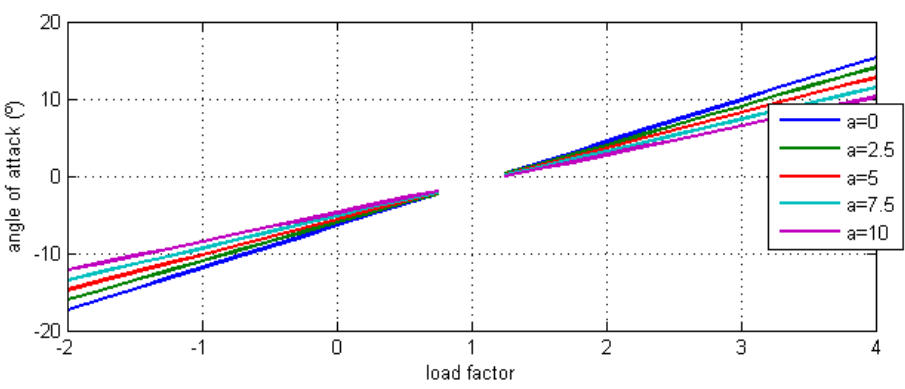

(b) Load factor sensitivity

Figure 5.4: Effect of $C_{L_{q}}$ on pull-up/push-over static characteristics

Table 5.1 summarises the results above, showing which derivatives/mass properties affect longitudinal dynamic and static characteristics significantly. It has been split in two for formatting purposes only.

This sensitivity analysis detected, at least, those parameters which, according to Roskam [17], are more relevant for the different dynamic characteristics.

There are empty columns on Table 5.1, meaning that some derivatives don't have a significant importance on any of the flying qualities parameters and are not worth studying for the purpose of this work. This is the case of $C_{L_{\dot{\alpha}}}$ and $C_{D_{\delta_{e}}}$.

$C_{D_{\alpha}}$ and $C_{L_{q}}$ only have significant effect on one parameter each, $\xi_{p h}$ and $\omega_{n_{p h}}$, respectively, but not as important as those of the other aerodynamic characteristics influencing these parameters. Therefore, possible changes in $C_{D_{\alpha}}$ and $C_{L_{q}}$ are also neglected in further studies.

We will then neglect four derivatives:

- $C_{L_{\dot{\alpha}}}$

- $C_{D_{\delta_{e}}}$

- $C_{D_{\alpha}}$

- $C_{L_{q}}$ 


\begin{tabular}{|c|ccccccc|}
\hline $\mathrm{FQ}$ & $C_{L_{\alpha}}$ & $C_{D_{\alpha}}$ & $C_{m_{\alpha}}$ & $C_{L_{q}}$ & $C_{m_{q}}$ & $C_{L_{\dot{\alpha}}}$ & $C_{m_{\dot{\alpha}}}$ \\
\hline \hline$\omega_{n_{p h}}$ & $\mathrm{x}$ & & $\mathrm{x}$ & $\mathrm{x}$ & $\mathrm{x}$ & & \\
$\xi_{p h}$ & $\mathrm{x}$ & $\mathrm{x}$ & $\mathrm{x}$ & & $\mathrm{x}$ & & \\
$\omega_{n_{s p}}$ & $\mathrm{x}$ & & $\mathrm{x}$ & & $\mathrm{x}$ & & \\
$\xi_{s p}$ & $\mathrm{x}$ & & $\mathrm{x}$ & & $\mathrm{x}$ & & $\mathrm{x}$ \\
$\delta_{e} \operatorname{vs} V$ & $\mathrm{x}$ & & $\mathrm{x}$ & & & & \\
$\alpha \operatorname{vs} V$ & $\mathrm{x}$ & & $\mathrm{x}$ & & & & \\
$\delta_{e} \operatorname{vs} n$ & $\mathrm{x}$ & & $\mathrm{x}$ & & $\mathrm{x}$ & & \\
$\alpha \operatorname{vs} n$ & $\mathrm{x}$ & & $\mathrm{x}$ & & $\mathrm{x}$ & & \\
\hline
\end{tabular}

\begin{tabular}{|c|ccccccc|}
\hline $\mathrm{FQ}$ & $C_{L_{\delta_{e}}}$ & $C_{D_{\delta_{e}}}$ & $C_{m_{\delta_{e}}}$ & $C_{D_{0}}$ & $C_{m_{0}}$ & $\theta_{\text {trim }}$ & $I_{y y}$ \\
\hline \hline$\omega_{n_{p h}}$ & & & & & & & \\
$\xi_{p h}$ & & & & $\mathrm{x}$ & & $\mathrm{x}$ & $\mathrm{x}$ \\
$\omega_{n_{s p}}$ & & & & & & & $\mathrm{x}$ \\
$\xi_{s p}$ & & & & & & & $\mathrm{x}$ \\
$\delta_{e} \operatorname{vs} V$ & & & $\mathrm{x}$ & $\mathrm{x}$ & $\mathrm{x}$ & \\
$\alpha \operatorname{vs} V$ & & $\mathrm{x}$ & & $\mathrm{x}$ & $\mathrm{x}$ & \\
$\delta_{e} \operatorname{vs} n$ & & $\mathrm{x}$ & & $\mathrm{x}$ & & & \\
$\alpha \operatorname{vs} n$ & $\mathrm{x}$ & & $\mathrm{x}$ & & & & \\
\hline
\end{tabular}

Table 5.1: Longitudinal flying qualities sensitivity to model parameters 
From this four derivatives, $C_{L_{q}}$ and $C_{L_{\dot{\alpha}}}$ are in the set of those where uncertainty is bigger (see page48). In particular, we had set $C_{L_{\dot{\alpha}}}$ to zero. The above analysis shows that there is no need to worry about possible errors on these derivatives.

\subsubsection{Lateral}

\section{Dynamic Characteristics}

Lateral dynamic characteristics under study only depend on the dynamic matrix. We test those parameters over which this matrix is built. Figures 5.5 and 5.7 illustrate longitudinal dynamic modes sensitivity to model parameters, whose domain of variation is represented in the $x$ axis by factors that multiply the baseline value, from -10 to 10 .

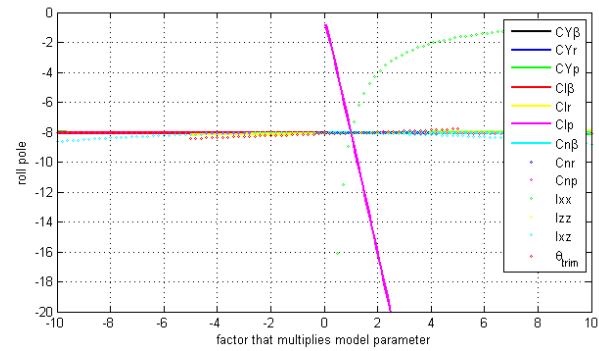

(a) Roll pole location

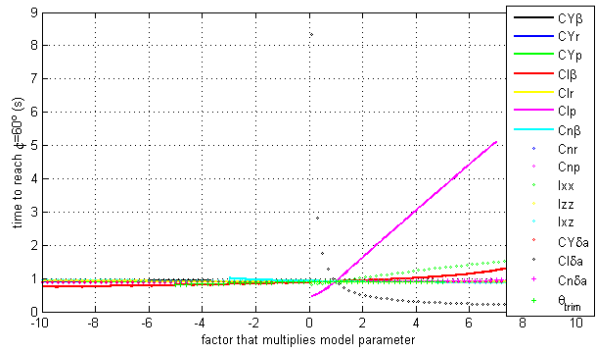

(b) Time to reach $\phi=60^{\circ}$

Figure 5.5: Roll performace sensitivity to model parameters

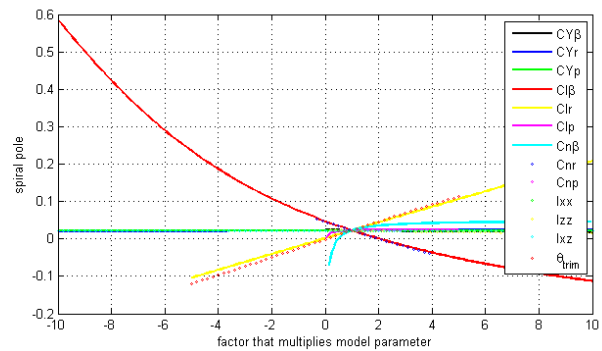

Figure 5.6: Spiral pole

\section{Static Characteristics}

Technically, it wouldn't be worth repeating this analysis for static characteristics, for the reason already mentioned in the longitudinal case. However, as Figures 5.8 to 5.11 show, some derivatives can be neglected in further studies ( $a$ is the factor by which each model parameter is multiplied).

Table 5.2 summarises the results for lateral FQ parameters, showing which derivatives/mass properties affect dynamic and static characteristics significantly. It has been split in two for formatting 


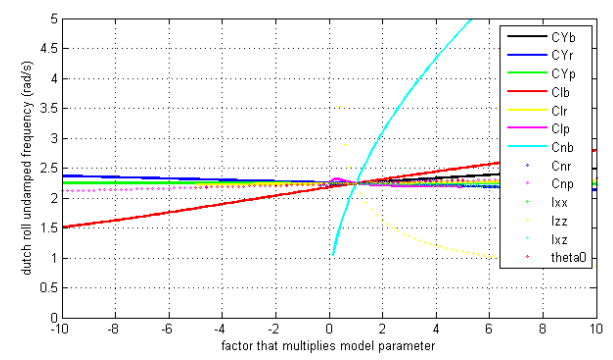

(a) Dutch roll undamped frequency

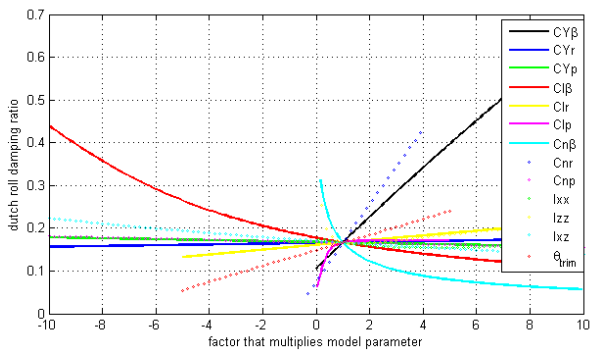

(b) Dutch roll damping ratio

Figure 5.7: Dutch roll sensitivity to model parameters

purposes only.

As in the longitudinal case, this sensitivity analysis detected, at least, those parameters which, according to Roskam [16], are more relevant for the different dynamic characteristics.

Empty columns on Table 5.2 show that some derivatives don't have a significant effect on any of the flying qualities parameters and therefore are not worth studying for the purpose of this work. This is the case of:

- $C_{Y_{p}}$

- $C_{Y_{r}}$

- $C_{n_{p}}$

- $C_{Y_{\delta_{a}}}$

- $C_{n_{\delta_{a}}}$

- $I_{x z}$

From the above model parameters, $C_{Y_{\delta_{a}}}$ and $C_{n_{\delta_{a}}}$ are in the set of those where uncertainty is bigger (see page 48). The above analysis shows that there is no need to worry about possible errors on these derivatives.

\subsubsection{Mass Properties Sensitivity to Design Parameters}

For this analysis, we model the aircraft with known and regular volumes like cylinders and parallelepipeds. This approach is valid because it accounts for the most important effects, and necessary because otherwise complexity would be prohibitive. Figure D.1, in Appendix D, shows the resultant aircraft, and also defines some important distances.

Based on this model, the contribution of the different components for inertia matrix is presented in Appendix D. Only those affected by the design parameters are considered: 


\begin{tabular}{|c|ccccccccc|}
\hline $\mathrm{FQ}$ & $C_{Y_{\beta}}$ & $C_{Y_{p}}$ & $C_{Y_{r}}$ & $C_{l_{\beta}}$ & $C_{l_{p}}$ & $C_{l_{r}}$ & $C_{n_{\beta}}$ & $C_{n_{p}}$ & $C_{n_{r}}$ \\
\hline \hline roll pole & & & & & $\mathrm{x}$ & & & & \\
$\left.t\right|_{\phi=60^{\circ}}$ & & & & & $\mathrm{x}$ & & & & \\
spiral pole & & & & $\mathrm{x}$ & & $\mathrm{x}$ & $\mathrm{x}$ & & $\mathrm{x}$ \\
$\omega_{n_{d r}}$ & & & & $\mathrm{x}$ & & & $\mathrm{x}$ & \\
$\xi_{d r}$ & $\mathrm{x}$ & & $\mathrm{x}$ & $\mathrm{x}$ & & $\mathrm{x}$ & \\
$\delta_{r}$ vs $\beta$ & & & & & & & $\mathrm{x}$ & \\
$\delta_{a}$ vs $\beta$ & & & & & & & $\mathrm{x}$ & \\
$\phi \operatorname{vs~} \beta$ & $\mathrm{x}$ & & & $\mathrm{x}$ & & & $\mathrm{x}$ & \\
\end{tabular}

\begin{tabular}{|c|cccccccccc|}
\hline $\mathrm{FQ}$ & $C_{Y_{\delta_{a}}}$ & $C_{l_{\delta_{a}}}$ & $C_{n_{\delta_{a}}}$ & $C_{Y_{\delta_{r}}}$ & $C_{l_{\delta_{r}}}$ & $C_{n_{\delta_{r}}}$ & $I_{x x}$ & $I_{z z}$ & $I_{x z}$ & $\theta_{\text {trim }}$ \\
\hline \hline roll pole & & & & & & & $\mathrm{x}$ & & \\
$\left.t\right|_{\phi=60^{\circ}}$ & & $\mathrm{x}$ & & & $\mathrm{x}$ & & & & \\
spiral pole & & & & & & & & & \\
$\omega_{n_{d r}}$ & & & & & & & & $\mathrm{x}$ & \\
$\xi_{d r}$ & & & & & & & & & $\mathrm{x}$ \\
$\delta_{r} \operatorname{vs} \beta$ & & & & & & & & & \\
$\delta_{a} \operatorname{vs} \beta$ & & & & $\mathrm{x}$ & & $\mathrm{x}$ & & & \\
$\phi \operatorname{vs} \beta$ & & $\mathrm{x}$ & & $\mathrm{x}$ & $\mathrm{x}$ & $\mathrm{x}$ & & & \\
\hline
\end{tabular}

Table 5.2: Lateral flying qualities sensitivity to model parameters 


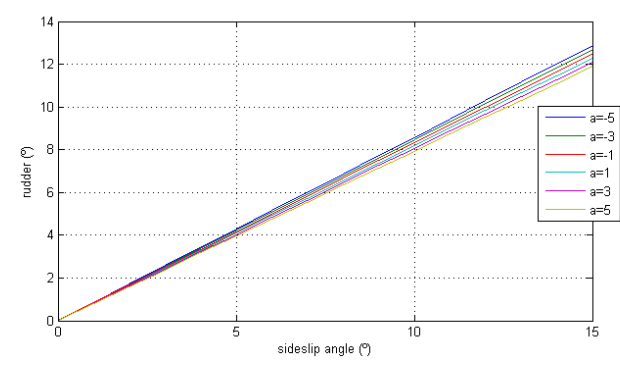

(a) Rudder position directional stability

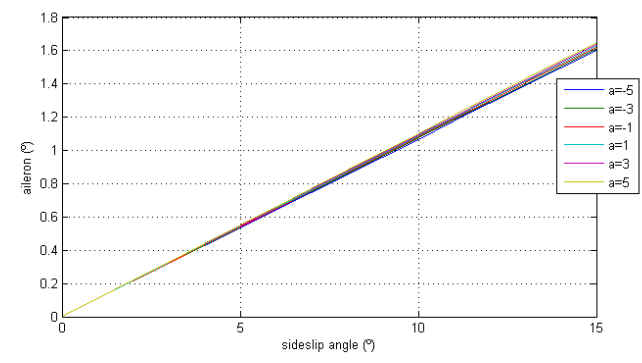

(b) Control-fixed dihedral effect

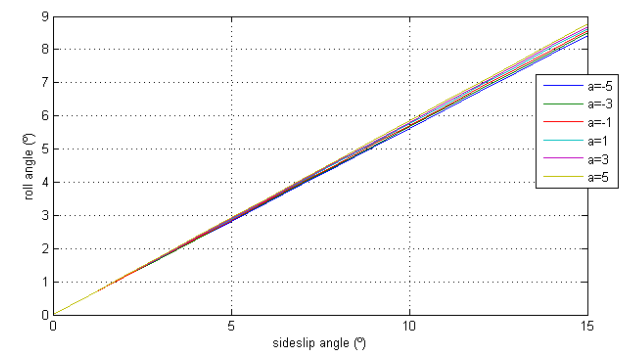

(c) Sideforce stability

Figure 5.8: Lateral static characteristics sensitivity to $C_{n_{\delta_{a}}}$

- Tail booms

- Elevator

- Vertical stabilizers and rudders

- Horizontal stabilizer and elevator

- Ailerons

Table 5.3 shows how to determine the changes in mass properties due to design parameters variation, expressed by variable $a$ in the following way:

$$
d^{\prime}=(1+a) d
$$

where $d$ is the design parameter baseline value and $d^{\prime}$ is the new one.

Some considerations on this Table 5.3 are worth mentioning:

- Booms are shells but here they are modeled as solid cylinders (it is simpler). Because $r \ll L$, this approximation is valid.

- In the above table, $m$ represents the mass of the corresponding component, not the mass of the whole aircraft. 


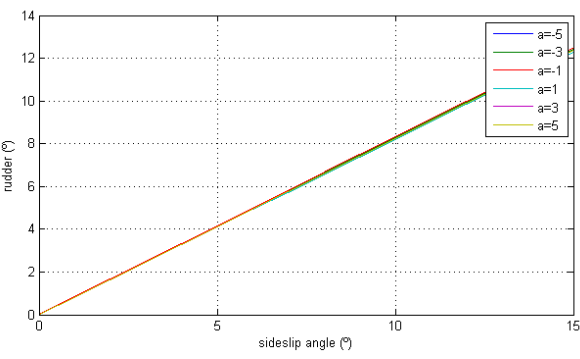

(a) Rudder position directional stability

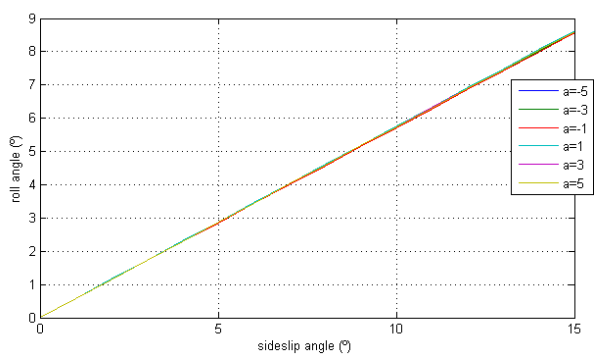

(b) Sideforce stability

Figure 5.9: Lateral static characteristics sensitivity to $C_{l_{\delta_{a}}}$

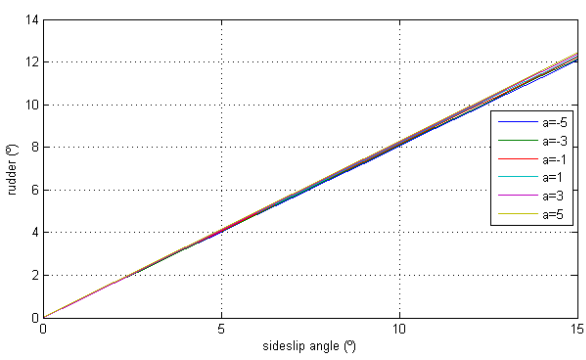

(a) Rudder position directional stability

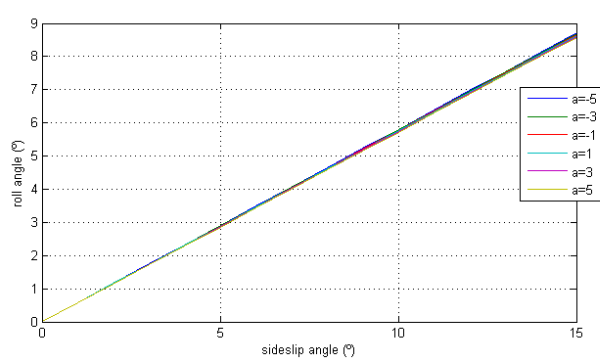

(b) Sideforce stability

Figure 5.10: Lateral static characteristics sensitivity to $C_{l_{\delta_{r}}}$

- It is possible to simplify the presented expressions by neglecting both $r$ and $w$, since they are very small relative to other dimensions.

\subsubsection{Aerodynamic Characteristics Sensitivity to Design Parameters}

Though it might be questionable if it can be considered an aerodynamic characteristic, we also include $\alpha_{\text {trim }}$ in this group.

For this analysis, we use the empiric expressions from Appendix B for three reasons:

- By looking at the expressions, it is easy to see which derivatives are more likely to be affected by the different design parameters and how they are affected

- They are not as time demanding as CFD simulations, allowing rapid computation of results for to different combinations of design parameters

- Tables 4.2 and 4.3 showed that they reasonably predicted CFD results. Even if only tail contribution is available, they can still be used because tail is the component most significantly affected by the chosen set of design parameters. 


\begin{tabular}{|c|c|c|c|}
\hline DP & $\begin{array}{l}\text { Affected } \\
\text { Comp. }\end{array}$ & Affected Param. & Effect on Mass Properties \\
\hline 1 & Booms & $d_{2}^{\prime}=(1+a) d_{2}$ & $\begin{array}{l}\Delta I_{x x}=m\left((2+a)^{2}-d_{2}^{2}\right) \\
\Delta I_{z z}=m\left((2+a)^{2}-d_{2}^{2}\right)\end{array}$ \\
\hline 1 & Rudd+VSt & $d_{2}^{\prime}=(1+a / 2) d_{2}$ & $\begin{aligned} \Delta I_{x x} & =m\left((2+a)^{2}-d_{2}^{2}\right) \\
\Delta I_{z z} & =m\left((2+a)^{2}-d_{2}^{2}\right)\end{aligned}$ \\
\hline 1 & Elev + HSt & $\begin{aligned} b^{\prime} & =(1+a) b \\
m^{\prime} & =(1+a) m\end{aligned}$ & $\begin{array}{l}\Delta I_{x x}=\frac{1}{12} m a\left(3 b^{2}+6 b^{2} a+4 b^{2} a^{2}+h^{2}+12 d_{1}^{2}\right) \\
\Delta I_{y y}=\frac{1}{12} m a\left(w^{2}+h^{2}+12 d_{1}^{2}+12 d_{4}^{2}\right) \\
\Delta I_{z z}=\frac{1}{12} m a\left(3 b^{2}+6 b^{2} a+4 b^{2} a^{2}+w^{2}+12 d_{4}^{2}\right)\end{array}$ \\
\hline 2 & Booms & $\begin{aligned} L^{\prime} & =(1+a) L \\
d_{3}^{\prime} & =\left(1+\frac{a}{2}\right) d_{3} \\
m^{\prime} & =(1+a) m\end{aligned}$ & $\begin{aligned} \Delta I_{x x} & =\frac{1}{2} a m\left(r^{2}+2 d_{1}^{2}+2 d_{2}^{2}\right) \\
\Delta I_{y y} & =\frac{1}{12} a m\left(3 L^{2}+3 L^{2} a+3 r^{2}+L^{2} * a^{2}+24 * d_{3}^{2}+15 d_{3}^{2} a+12 d_{1}^{2}+3 d_{3}^{2} a^{2}\right) \\
\Delta I_{z z} & =\frac{1}{12} a m\left(3 L^{2}+3 L^{2} a+3 r^{2}+L^{2} * a^{2}+24 * d_{3}^{2}+15 d_{3}^{2} a+12 d_{2}^{2}+3 d_{3}^{2} a^{2}\right)\end{aligned}$ \\
\hline 2 & Rudd+VSt & $d_{4}^{\prime}=(1+a) d_{4}$ & $\begin{aligned} \Delta I_{y y} & =m d_{4}^{2} a(2+a) \\
\Delta I_{z z} & =m d_{4}^{2} a(2+a)\end{aligned}$ \\
\hline 2 & Elev+HSt & $d_{4}^{\prime}=(1+a) d_{4}$ & $\begin{aligned} \Delta I_{y y} & =m d_{4}^{2} a(2+a) \\
\Delta I_{z z} & =m d_{4}^{2} a(2+a)\end{aligned}$ \\
\hline 6 & Rudd+VSt & $\begin{aligned} b^{\prime} & =(1+a) b \\
m^{\prime} & =(1+a) m\end{aligned}$ & $\begin{array}{l}\Delta I_{x x}=\frac{1}{12} m a\left(3 b^{2}+3 b^{2} a+b^{2} a^{2}+h^{2}+12 d_{1}^{2}+12 d_{2}^{2}\right) \\
\Delta I_{y y}=\frac{1}{12} m a\left(3 b^{2}+3 b^{2} a+b^{2} a^{2}+w^{2}+12 d_{1}^{2}+12 d_{4}^{2}\right) \\
\Delta I_{z z}=\frac{1}{12} m a\left(w^{2}+h^{2}+12 d_{4}^{2}+12 d_{2}^{2}\right)\end{array}$ \\
\hline 8 & Elev+HSt & $d_{1}^{\prime}=(1+a) d_{1}$ & $\begin{array}{l}\Delta I_{x x}=\operatorname{mad}_{1}^{2}(2+a) \\
\Delta I_{y y}=\operatorname{mad}_{1}^{2}(2+a)\end{array}$ \\
\hline
\end{tabular}

Table 5.3: Effect on mass properties 


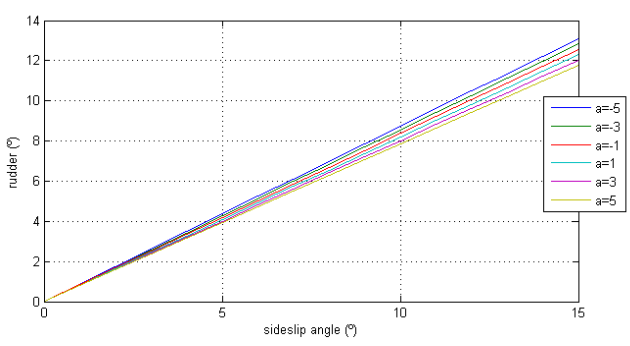

(a) Rudder position directional stability

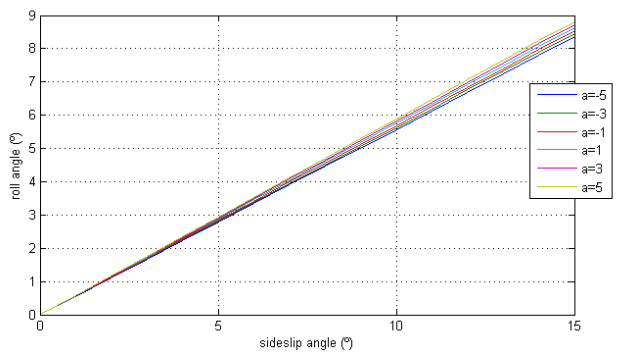

(b) Sideforce stability

Figure 5.11: Lateral static characteristics sensitivity to $C_{l_{\beta}}$

DATCOM [10] is an extensive and very detailed compendium of correlations for aerodynamic characteristics of some aircraft configuration, based on wind tunnel tests. Roskam [17] presents some of DATCOM results for the most important derivatives, in a more easy-to-consult way. By looking at the different expressions, it is possible to verify their sensitiveness to the design parameters of Section 5.2.

Expressions in Appendix B are already particularised for ANTEX, and terms likely to be affected by the design parameters (like the ones reflecting stabilizers and control surfaces contributions) are presented in a more detailed way.

Because of its inherent complexity, effects on drag are handled separately. Preceding section showed that influence of drag was only relevant for phugoid damping ratio, and was mainly due to its trim value. According to equation 4.1, trim drag coefficient is given by:

$$
C_{D_{\text {trim }}}=C_{D_{0}}+f\left(\alpha_{\text {trim }}\right)+g\left(\delta_{e_{\text {trim }}}\right)
$$

For simplicity, we assume that the chosen design parameters affect essencially the first term, $C_{D_{0}}$, and that possible variation on the remaining two compensate for each other. This term is usually known as parasitic drag coefficient

Though some correlations exist for $C_{D_{0}}$ determination (see e.g. [10]), it is not so straightforward to compute it. However, it is known to be directly proportional to surface area exposed to airflow (also known as total wetted area), because it has its source on pressure distribution and shear stress on the wall, which also has pressure dimension. Effects on $C_{D_{0}}$ will, then be analysed from a qualitative point of view: if total area increases (decreases), $C_{D_{0}}$ also increases (decreases). This information is, by itself, very valuable - any decrease in drag is almost always welcome (if other characteristics considered more important for the mission are not seriously degraded as a result).

Design parameters that directly affect total surface area are 1,2 and 6 . The amount of area change due to each of the parameters is left for Section 5.4.

Considering now the other aerodynamic characteristics, and looking at the empiric expressions, it is 


\begin{tabular}{|c|ccccc|}
\hline Design Parameter & $A$ & $S_{h}$ & $\frac{\partial \epsilon}{\partial \alpha}$ & $l_{h}$ & $c_{e l}$ \\
\hline 1 & $\mathrm{x}$ & $\mathrm{x}$ & & & \\
2 & & & $\mathrm{x}$ & $\mathrm{x}$ & \\
3 & & & & & $\mathrm{x}$ \\
8 & & & & & \\
\hline
\end{tabular}

Table 5.4: Longitudinal elements affected by design parameters

\begin{tabular}{|c|c|c|c|c|c|c|c|c|c|}
\hline Design Parameter & $l_{v}$ & $A_{v}$ & $S_{v}$ & $c_{\text {rud }}$ & $c_{a i l}$ & $\eta_{i}$ & $b_{h}$ & $A_{h}$ & $S_{h}$ \\
\hline 1 & & & & & \multirow{6}{*}{$\mathrm{x}$} & & $\mathrm{x}$ & $\mathrm{x}$ & $\mathrm{x}$ \\
\hline 2 & $\mathrm{x}$ & & & & & \multirow{5}{*}{$\mathrm{x}$} & & \multirow{5}{*}{$\mathrm{x}$} & \multirow{5}{*}{$\mathrm{x}$} \\
\hline 4 & & & & & & & $\mathrm{x}$ & & \\
\hline 5 & & & & & & & & & \\
\hline 6 & & $\mathrm{x}$ & $\mathrm{x}$ & & & & & & \\
\hline 7 & & & & $\mathrm{x}$ & & & & & \\
\hline
\end{tabular}

Table 5.5: Lateral elements affected by design parameters

possible to identify a list of some variables, not necessarily independent, directly related to the design parameters. Tables 5.4 and 5.4 show which of those variables depend on.

Using this information, it is easier to see which aerodynamic characteristics are affected and how they are affected (see Tables 5.6 and 5.7).

\subsection{Effect of Design Parameters on Flying Qualities}

Flying qualities dependence on the design variables is finally studied, using the knowledge acquired in the the previous sections. For that, we apply changes of $\pm 20 \%$ to each design parameter, independently, according to equation (5.1). Then, we determine the new model parameters (see Appendix F, introduce them in the dynamic model and, finally, we use the linear decoupled model to and equations (3.1) to (3.7) to check the effect on flying qualities.

If the above changes are to be applied to an existing aircraft, with acceptable, but maybe improvable, characteristics, a limit of $\pm 20 \%$ is over what would be considered reasonable. The purpose of using such high limit is only to guarantee that it boxes all the plausible alterations.

Tables 5.8 and 5.9 show the variation in dynamic longitudinal and lateral flying qualities for $a=$ \pm 0.2 (only changes above $1 \%$ are considered, and only those parameters influencing FQ are presented). It would probably be lighter and clearer for the reader if only qualitative effects were presented, but there are at least two major advantages in quantifying them:

- For the same variation, it is possible to see which design parameters are more influent 


\begin{tabular}{|c|c|c|}
\hline Design Parameter & Affected Parameters & Effect on Derivatives (Absolute Variation) \\
\hline 1 & $\begin{array}{l}S_{h}^{\prime}=(1+a) S_{h} \\
A_{h}^{\prime}=(1+a) A_{h}\end{array}$ & $\begin{aligned} \Delta C_{L_{\alpha}} & =\frac{S_{h}}{S}\left(1-\frac{\partial \epsilon}{\partial \alpha}\right)\left[(1+a) C_{L_{\alpha_{h}}}||_{A_{h}^{\prime}}-\left.C_{L_{\alpha_{h}}}\right|_{A_{h}}\right] \\
\Delta C_{m_{\alpha}} & =-\Delta C_{L_{\alpha}} \frac{l_{h}}{c} \\
\Delta C_{m_{q}} & =-2 \frac{\Delta C_{L_{\alpha}}}{\left(1-\frac{\partial \epsilon}{\partial \alpha}\right)}\left(\frac{l_{h}}{c}\right)^{2} \\
\Delta C_{m_{\dot{\alpha}}} & =\Delta C_{m_{q}} \frac{\partial \epsilon}{\partial \alpha} \\
\Delta C_{L_{\delta_{e}}} & =\frac{1.3187 C_{l_{\delta_{e}}}}{2 \pi\left(1+\frac{t_{h}}{c_{h}}\right)} \frac{\Delta C_{L_{\alpha}}}{\left(1-\frac{\partial \epsilon}{\partial \alpha}\right)} \\
\Delta C_{m_{\delta_{e}}} & =-\Delta C_{L_{\delta_{e}}} \frac{l_{h}}{c} \\
\Delta C_{m_{0}} & =-\frac{\Delta C_{L_{\alpha}}}{\left(1-\frac{\partial \epsilon}{\partial \alpha}\right)} \frac{l_{h}}{c}\left(i_{t}+\epsilon_{0}\right)\end{aligned}$ \\
\hline 2 & $l_{h}^{\prime}=(1+a) l_{h}$ & $\begin{aligned} \Delta C_{L_{\alpha}} & =-C_{L_{\alpha_{h}}} \frac{S_{h}}{S}\left(\left.\frac{\partial \epsilon}{\partial \alpha}\right|_{l_{h}^{\prime}}-\left.\frac{\partial \epsilon}{\partial \alpha}\right|_{l_{h}}\right) \\
\Delta C_{m_{\alpha}} & =-\frac{C_{L_{\alpha_{h}}} l_{h} S_{h}}{c S}\left[(1+a)\left(1-\left.\frac{\partial \epsilon}{\partial \alpha}\right|_{l_{h}^{\prime}}\right)-\left(1-\frac{\partial \epsilon}{\partial \alpha} l_{h}\right)\right] \\
\Delta C_{m_{q}} & =-2 \frac{C_{L_{\alpha_{h}}} S_{h}}{S} \frac{l_{h}^{2}}{c^{2}}\left[(1+a)^{2}-1\right] \\
\Delta C_{m_{\dot{\alpha}}} & =-2 \frac{C_{L_{\alpha_{h}}} S_{h}}{S} \frac{l_{h}^{2}}{c^{2}}\left[\left.(1+a)^{2} \frac{\partial \epsilon}{\partial \alpha}\right|_{l_{h}^{\prime}}-\left.\frac{\partial \epsilon}{\partial \alpha}\right|_{l_{h}}\right] \\
\Delta C_{m_{\delta_{e}}} & =-a C_{L_{\delta_{e}}} \frac{l_{h}}{c} \\
\Delta C_{m_{0}} & =-a C_{L_{\alpha_{h}}} \frac{l_{h} S_{h}}{c S}\left(i_{t}+\epsilon_{0}\right)\end{aligned}$ \\
\hline 3 & $c_{e l}^{\prime}=(1+a) c_{e l}$ & $\begin{aligned} \Delta C_{L_{\delta_{e}}} & \left.\left.=\left.\frac{1.3187 C_{L_{\alpha_{h}}}}{2 \pi\left(1+\frac{t_{h}}{c^{h}}\right)} \frac{S_{h}}{S}\left(C_{\delta_{\delta_{e}}}\right]\right|_{c_{e l}^{\prime}}-C_{l_{\delta_{e}}}\right]\left.\right|_{c_{e l}}\right) \\
\Delta C_{m_{\delta_{e}}} & =-\Delta C_{L_{\delta_{e}}} \frac{l_{h}}{c}\end{aligned}$ \\
\hline 4 & $h_{h}^{\prime}=a$ & $\begin{aligned} \Delta C_{L_{\alpha}} & =C_{L_{\alpha_{h}}} \frac{S_{h}}{S}\left(\left.\frac{\partial \epsilon}{\partial \alpha}\right|_{h_{h}=0}-\left.\frac{\partial \epsilon}{\partial \alpha}\right|_{h_{h}^{\prime}}\right) \\
\Delta C_{m_{\alpha}} & =-\Delta C_{L_{\alpha}} \frac{l_{h}}{c}\end{aligned}$ \\
\hline
\end{tabular}

Table 5.6: Effect of design changes on longitudinal derivatives 


\begin{tabular}{|c|c|c|}
\hline Design Parameter & Affected Parameters & Effect on Derivatives (Absolute Variation) \\
\hline 1 & $\begin{aligned} b_{h}^{\prime} & =(1+a) b_{h} \\
A_{h}^{\prime} & =(1+a) A_{h} \\
S_{h}^{\prime} & =(1+a) S_{h}\end{aligned}$ & $\Delta C_{l_{p}}=0.5 \frac{S_{h}}{S}\left(\frac{b_{h}}{b}\right)^{2}\left[\left.(1+a)^{3}\left(C_{l_{p}}\right)_{h}\right|_{A_{h}^{\prime}}-\left.\left(C_{l_{p}}\right)_{h}\right|_{A_{h}}\right]$ \\
\hline 2 & $l_{v}^{\prime}=(1+a) l_{v}$ & $\begin{aligned} \Delta C_{n_{\beta}} & =-a C_{Y_{\beta}} \frac{l_{v}}{b} \\
\Delta C_{l_{r}} & =-2 a C_{Y_{\beta}} \frac{z_{v} l_{v}}{b^{2}} \\
\Delta C_{n_{r}} & =-2 C_{Y_{\beta}}\left(\frac{l_{v}}{b}\right)^{2}\left[(1+a)^{2}-1\right] \\
\Delta C_{n_{\delta_{r}}} & =-a C_{Y_{\delta_{r}}} \frac{l_{v}}{b}\end{aligned}$ \\
\hline 4 & $c_{a i l}^{\prime}=(1+a) c_{a i l}$ & $\Delta C_{l_{\delta_{a}}}=\frac{0.7927}{\pi}\left(\frac{\beta C_{l_{\delta}}^{\prime}}{k}\right)\left(\left.C_{l_{\delta_{\text {theory }}}}\right|_{c_{a i l}^{\prime}}-\left.C_{l_{\delta_{\text {theory }}}}\right|_{c_{a i l}}\right)$ \\
\hline 5 & $\eta_{i}^{\prime}=(1+a) \eta_{i}$ & $\Delta C_{l_{\delta_{a}}}=\frac{0.7927}{\pi} C_{l_{\delta_{\text {theory }}}}\left[\left.\left(\frac{\beta C_{l_{\delta}}^{\prime}}{k}\right)\right|_{\eta_{i}^{\prime}}-\left.\left.\left(\frac{\beta C_{l_{\delta}}^{\prime}}{k}\right)\right|_{\eta_{i}^{\prime}} k\right|_{\eta_{i}}\right]$ \\
\hline 6 & $\begin{aligned} b_{v}^{\prime} & =(1+2 a) b_{v} \\
A_{v}^{\prime} & =(1+2 a) A_{v} \\
S_{h}^{\prime} & =(1+2 a) S_{h}\end{aligned}$ & $\begin{aligned} \Delta C_{Y_{\beta}} & =-2\left(\frac{C_{Y_{\beta_{w b h}}}}{C_{Y_{\beta_{v_{e} f f}}}}\right) \frac{S_{v}}{S}\left[(1+a) C_{Y_{\beta_{v_{e} f f}}}\left|A_{A_{v}^{\prime}}-C_{Y_{\beta_{v_{e} f f}}}\right| A_{v}\right] \\
\Delta C_{l_{\beta}} & =\Delta C_{Y_{\beta}} \frac{z_{v}}{b} \\
\Delta C_{n_{\beta}} & =-\Delta C_{Y_{\beta}} \frac{l_{v}}{b} \\
\Delta C_{l_{p}} & =\Delta C_{Y_{\beta}}\left(\frac{z_{v}}{b}\right)^{2} \\
\Delta C_{l_{r}} & =-2 \Delta C_{Y_{\beta}} \frac{z_{v} l_{v}}{b^{2}} \\
\Delta C_{n_{r}} & =-2 \Delta C_{Y_{\beta}}\left(\frac{l_{v}}{b}\right)^{2} \\
\Delta C_{Y_{\delta_{r}}} & =2.17 \alpha_{\delta_{\delta_{l}}} \frac{S_{v}}{S}\left[(1+a) C_{L_{\alpha_{v}}}\left|A_{h}^{\prime}-C_{L_{\alpha_{v}}}\right| A_{h}\right] \\
\Delta C_{l_{\delta_{r}}} & =\Delta C_{Y_{\delta_{r}}} \frac{z_{v}}{b} \\
\Delta C_{n_{\delta_{r}}} & =-\Delta C_{Y_{\delta_{r}}} \frac{l_{v}}{b}\end{aligned}$ \\
\hline 7 & $c_{r u d d}^{\prime}=(1+a) c_{r u d d}$ & $\begin{aligned} \Delta C_{Y_{\delta_{r}}} & \left.=\left.2.17 C_{L_{\alpha_{v}}} \alpha_{\delta_{C_{l}}}\right|_{c_{r u d d}^{\prime}}-\left.\alpha_{\delta_{C_{l}}}\right|_{c_{r u d d}}\right) \\
\Delta C_{l_{\delta_{r}}} & =\Delta C_{Y_{\delta_{r}}} \frac{z_{v}}{b} \\
\Delta C_{n_{\delta_{r}}} & =-\Delta C_{Y_{\delta_{r}}} \frac{l_{v}}{b}\end{aligned}$ \\
\hline
\end{tabular}

Table 5.7: Effect of design changes on lateral derivatives 
- Quantification gives cues on the expectable changes in flying qualities

\section{Longitudinal}

\begin{tabular}{|c|c|c|c|c|c|}
\hline \multirow{2}{*}{ Design Parameters } & \multicolumn{4}{|c|}{ Flying Qualities } \\
\cline { 3 - 6 } & $\omega_{n_{p h}}$ & $\xi_{p h}$ & $\omega_{n_{s p}}$ & $\xi_{s p}$ \\
\hline \multirow{2}{*}{1} & $a=0.2$ & -2 & 3.8 & 9.7 & 4.7 \\
& $a=-0.2$ & 2.5 & -5.7 & -10.4 & -5.4 \\
\hline \multirow{2}{*}{2} & $a=0.2$ & -4.5 & 6.3 & 5.2 & 7.5 \\
& $a=-0.2$ & 5 & -7 & -6.5 & -8 \\
\hline
\end{tabular}

Table 5.8: Effects of a $\pm 20 \%$ change in design parameters on longitudinal flying qualites

As mentioned in the beginning of this chapter, we used lateral and longitudinal linear models for parametric studies. However, Chapter 4 showed some deficiencies on phugoid damping ratio and spiral stability prediction. Therefore, it is important to verify the real variation for $\xi_{p h}$ and $T_{2_{\text {spiral }}}$, using the linearised and nonlinear models, respectively. We test only those parameters that most influence phugoid and spiral (1,2 and 6$)$, and for $a=0.2$. Table 5.10 summarises the results obtained: variations are on the same direction as those predicted using the linear decoupled model and, contrary to what Table 5.9 shows, parameter 1 has some influence on spiral stability..

For static characteristics, and since they are represented by curves, effects are better understood if presented graphically. Results for those characteristics varying significantly can be found on Figures $5.12(\mathrm{a})$ and 5.12 .

Although not being a flying quality, it is also important to measure, at least qualitatively, how drag is affected. Table 5.11 shows absolute variation on total area due to a $20 \%$ change in design parameters. If the change is negative, drag decreases.

From the above results it is possible to draw important conclusions. Up to $20 \%$ of absolute change:

- Effects due to parameter 8 are negligible

- Parameters $3,4,5,7$ and 8 don't affect drag component due to surface area

- Parameter 3 only affects longitudinal FQ parameters

- Parameters 4,5,6 and 7 only affect lateral FQ parameters

- None of the parameters have a significant effect on roll time constant

\subsection{Applicability to Other Aircraft}

We claimed that, by performing parametric studies in two steps, conclusions taken from the first one could be applicable to other aircraft types besides our UAV. 


\begin{tabular}{|c|c|c|c|c|c|c|}
\hline \multirow{2}{*}{ Design Parameters } & \multicolumn{5}{|c|}{ Flying Qualities } \\
\cline { 2 - 7 } & $\tau_{\text {roll }}$ & $\left.t\right|_{\phi=60^{\circ}}$ & $T_{2_{\text {spiral }}}$ & $\omega_{n_{d r}}$ & $\xi_{d r}$ \\
\hline \multirow{2}{*}{2} & $a=0.2$ & & & 5.6 & 11.6 & 9.6 \\
& $a=-0.2$ & & & -1.3 & -14.7 & -6 \\
\hline \multirow{2}{*}{4} & $a=0.2$ & & -8.3 & & & \\
& $a=-0.2$ & & 9.9 & & & \\
\hline \multirow{2}{*}{5} & $a=0.2$ & & -16.5 & & & \\
& $a=-0.2$ & & 24.2 & & & \\
\hline \multirow{2}{*}{6} & $a=0.2$ & & & -10.5 & 22.3 & 3.6 \\
& $a=-0.2$ & & & 44.6 & -35.8 & 1.8 \\
\hline
\end{tabular}

Table 5.9: Effects of a $\pm 20 \%$ change in design parameters on lateral flying qualities

\begin{tabular}{|c|c|c|}
\hline Design Parameter & $\xi_{p h}$ & $T_{2_{\text {spiral }}}$ \\
\hline \hline 1 & $5.9 \%$ & $11.1 \%$ \\
2 & $9.4 \%$ & $27.5 \%$ \\
6 & & $-10 \%$ \\
\hline
\end{tabular}

Table 5.10: Effects of a $20 \%$ change in design parameters on phugoid and spiral, using the linearised and nonlinear models

\begin{tabular}{|c|c|c|}
\hline Design Parameter & Total Area Change $\left(m^{2}\right)$ & $C_{D_{0}}$ \\
\hline \hline 1 & 0.189 & \\
2 & 0.0132 & increase \\
6 & 0.132 & \\
\hline
\end{tabular}

Table 5.11: Effects of a $20 \%$ change in design parameters on drag 


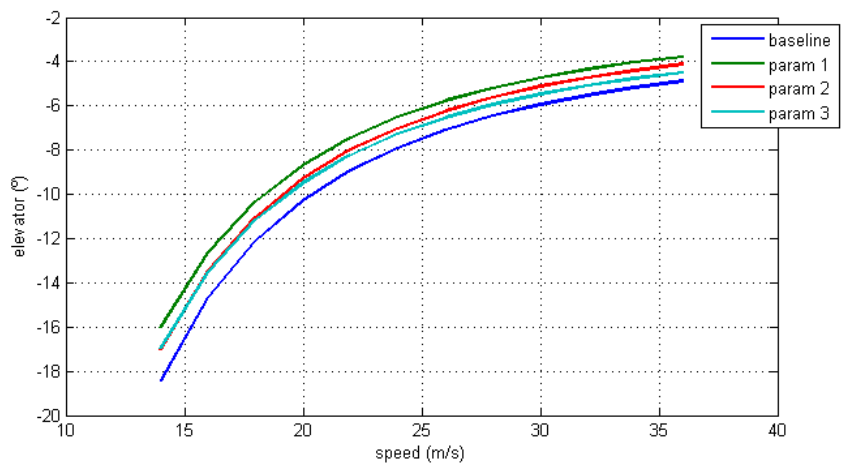

(a) Elevator position stability

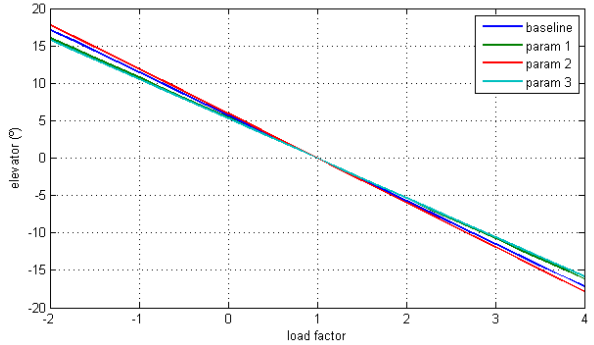

(b) Elevator per $n$

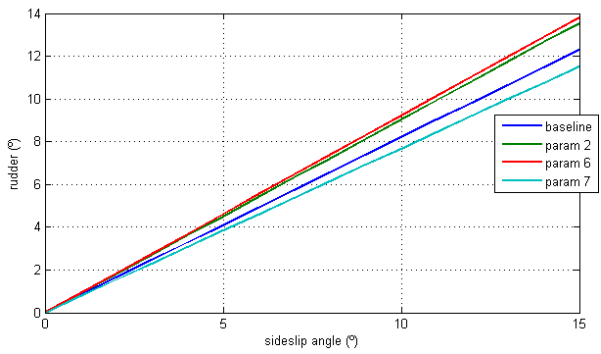

(c) Rudder position directional stability

Figure 5.12: Effect of a $20 \%$ change in design parameters on static characteristics

The tool developed to study flying qualities sensitivity to model parameters is certainly valid for other configurations, since it is based on the general form of the 6-DOF dynamic mode for an aircraft. However, we can not state that the same applies for the obtained results without making some tests.

Therefore, we used the same procedure to check different aircraft results. We could simply check if the ones we got agreed with those from existing sensitivity analysis, as explained on page 71 , but because they are presented in a different manner, difficulting proper interpretation, we decided to move on with new tests.

We analysed two other airplanes, different in size,configuration and mission, whose characteristics can be found in Roskam [17]. We used the same baseline condition as for ANTEX, cruise, and assured that they had a conventional response, that is to say, conventional dynamic modes: phugoid, short period, roll, spiral and dutch roll modes. The aircraft tested were:

Aircraft 1: representative of a small, single pisto-engine general aviation airplane such as the Cessna 182 (see Figure 5.13) - corresponds to Roskam's airplane A.

Aircraft 2: representative of a small, regional, twin-turboprop commuter airplane such as the Beech 99 (see Figure 5.14) - corresponds to Roskam's airplane E. 


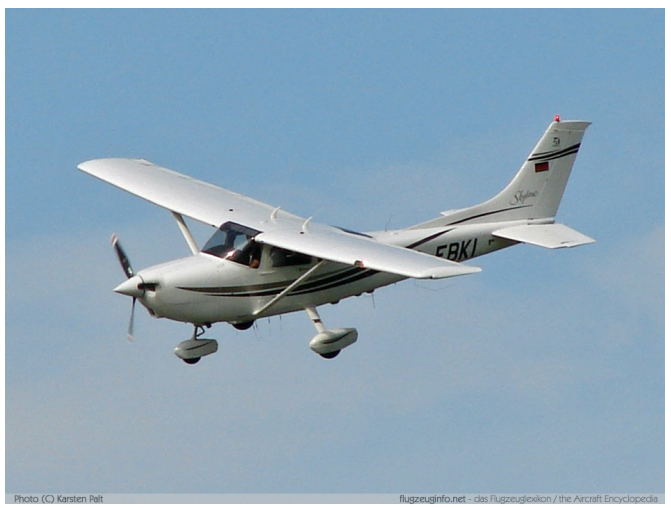

Figure 5.13: Cessna 182. Reprinted from http://www.flugzeuinfo.net

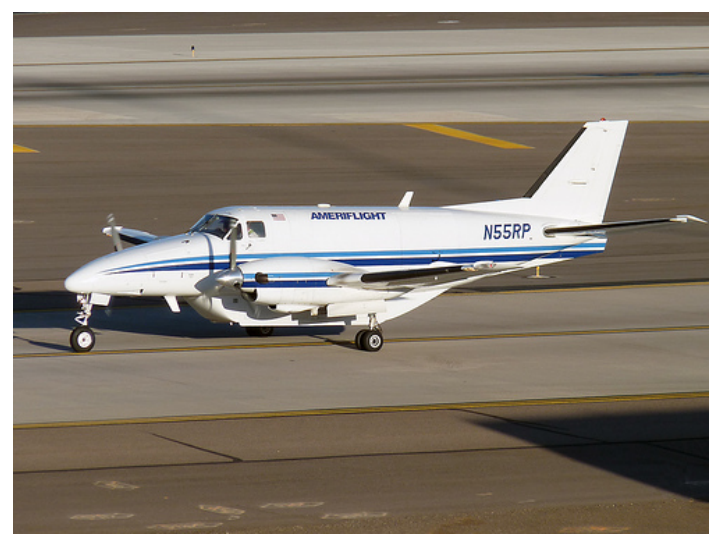

Figure 5.14: Beech 99. Reprinted from http://gallery.photo.net

The results obtained for each aircraft can be found in Appendix E. Comparing to the ones for ANTEX, similarity is evident. However, looking at the result for dutch roll damping ratio of Aircraft 2, $I_{x z}$ effect is similar, but more acentuaded and, consequently, not neglectable. This is due to the high baseline value for this product of inertia.

Nevertheless, we can say that Appendix E results validate the applicability of conclusions from step one (Section 5.3) to other aircraft configurations. 


\section{Chapter 6}

\section{Improving ANTEX-M X03 Design}

On the previous chapter, it was possible to see how the various flying qualities parameters of a specific UAV responded to changes in pre-defined design variables. Not only an important feeling of the resulting effects was acquired: they were actually quantified. In fact, this was the general objective of this project, as presented in Section 1.1. It is now possible to make specific studies regarding the improvement of some of the flying qualities parameters, most probably at the expense of others.

The purpose of this chapter is then to exemplify how the information gained before can be used to modify the aircraft response in order to make it more suitable for its design objectives. Based on current flying qualities and regarding a specific mission, some design changes are introduced aiming the improvement of some characteristics, but, above all, the improvement of global operational performance.

It is important to emphasise, however, that the solution presented is not optimal. In fact, optimization is the ultimate end for the tool herein developed, parametric studies from Chapter 5 , but it falls outside the scope of this work.

\subsection{Studying a New Design}

The starting point for this study is an existing UAV which has already been tested in flight and whose current flying qualities, determined on Chapter 4, are, according to piloted criteria, level 1, that is to say, clearly adequate for the mission flight phase. But that does not mean that they can't be improved, and this is certainly something worth analysing, if there is a well defined and objective strategy to follow.

Any design study should be performed according to a checklist similar to the one below.

1. Define the objective 
2. Write it as a set of requirements

3. Lay on possible restrictions

4. Make a list of parameters to improve

5. Introduce design changes accordingly

6. Check the consequences: was improvement achieved? At the expense of what?

In order to find a good solution, this should be an iterative process. But that is not the goal of this chapter. Instead, the intention is to show that slight modifications to current design can, indeed, make the aircraf more suitable to some mission.

\subsection{Problem Definition}

\subsubsection{Mission}

One of the missions of most interest to the PoAF, at least at the moment, is surveillance. This is, in fact, the type of task that has captured the attention of different entities to UAV development in PoAF.

This kind of mission asks for certain aircraft characteristics like low speed flight and stability. Low speed is already common in UAVs and will not be given much attention to.

Stability is related to the ability of the aircraft to mantain a certain flight condition and to perform a smooth, not necessarily slow, transition from one flight condition to another. The first is related to possible disturbances, like gusts, and the second to deliberate inputs. In both cases, it is desirable that any existing oscillations subside quickly. This is directely related to an adequate damping ratio of the different modes, whether they are short or long term modes. Additionally, slopes of static characteristics curves, especially elevator-related ones, shall not be too smooth, so that undeliberate situations don't easily lead to a different flight condition.

\subsubsection{Restrictions}

While studying possible modifications to ANTEX design, there are some restrictions to take into account:

1. The aircraft must be statically stable

2. Natural modes which are stable ought to remain stable

3. Spiral unstability shall not get worse

4. Drag penalties are to be avoided 
Items 1 to 3 are more safety-oriented while the last one is more related to costs/efficiency, and that is why it is given so much importance. Never desregarding safety, which is tighter for piloted aircraft, drag minimization is a very important issue, as it dictates energy/fuel consumption and, consequentely, efficiency. Less fuel consumption means, for the same amount of fuel, longer endurance and range. On the other hand, if endurance and range are held constant, extra fuel can be traded by extra payload. These are definately desirable characteristics for every aircraft, no matter the mission they were designed for, nor if they are piloted or not.

\subsection{Analysis of a Design Option}

Now that both ojective and restrictions are clearly defined, it is time to choose a set of design changes and study the effects on flying qualities.

\section{Goal}

- increase phugoid and dutch roll damping ratios

- increase the low speed margin for elevator deflection

Short period damping ratio and spiral time to double amplitude have quite acceptable values, clearly above the inferior limits for a level 1 rating, and improvement of these characteristics is not a specific goal. As to the second item, it is not specifically related to surveillance, but it is definitely something worth looking at,irrespective of the mission, as mentioned on Chapter $4^{1}$.

\subsubsection{Design Modifications}

Though not optimal, the design option presented is based on a careful analysis of results from Section 5.4.

- $+5 \%$ parameter 1: increase of horizontal tail span

- $+10 \%$ parameter 2: increase of tail booms lenght

- $+10 \%$ parameter 3: increase of elevator chord

- $-10 \%$ parameter 6: decrease of vertical tail span

\subsubsection{Results}

In order to determine the different results (dynamic ones) we used the linearised model. For parametric studies it was important to use a simpler approach, but in this case it is not so relevant.

\footnotetext{
${ }^{1}$ The low speed area is very important for take-off and landing, which are not addressed to on this project.
} 
Additionally, for phugoid damping ratio prediction, we saw that the linear decoupled model was not accurate. For spiral stability, though, we ran a nonlinear simulation.

\section{Goal Parameters:}

\begin{tabular}{|c|c|}
\hline$\xi_{p h}$ & $\xi_{d r}$ \\
\hline \hline$+7 \%$ & $+2.9 \%$ \\
\hline
\end{tabular}

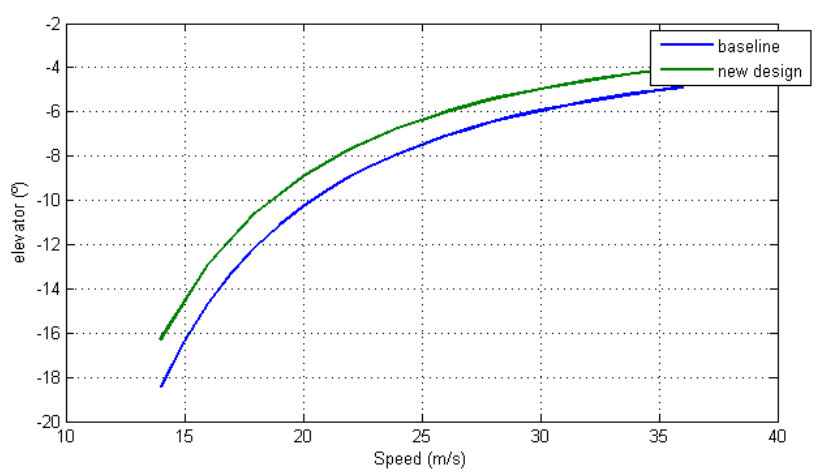

Figure 6.1: Effect of design changes on elevator position stability

Results show that the chosen design changes improve our goal parameters.

\section{Restriction Parameters:}

\begin{tabular}{|c|c|}
\hline$T_{2_{\text {spiral }}}(s)$ & Total Area Change $\left(\mathrm{m}^{2}\right)$ \\
\hline \hline$+49.3 \%$ & -0.0122 \\
\hline
\end{tabular}

The above table shows that spiral stability did not worsen; in fact, we managed to increase it. As to drag penalties, we can only say that, by reducing the total wetted area, we also reduced parasitic drag. Area reduction contributes to drag reduction, but other factors not studied here might have an adverse effect. In order to understand the real effect on drag, CFD should be used. However, introducing the design changes on the CFD model is not so straightforward, and simulations require considerable time.

Nevertheless, results here presented are still very valuable.

\section{Remaining:}

Though they are not the focus of this design study, remaining FQ will certainly vary. Table below presents this variation for dynamic parameters, and Figure 6.2 for static characteristics (we only consider affected FQ parameters).

\begin{tabular}{|c|c|c|c|}
\hline$\omega_{n_{p h}}$ & $\omega_{n_{s p}}$ & $\xi_{s p}$ & $\xi_{d r}$ \\
\hline \hline$-2.6 \%$ & $6 \%$ & $5.2 \%$ & $-6.2 \%$ \\
\hline
\end{tabular}




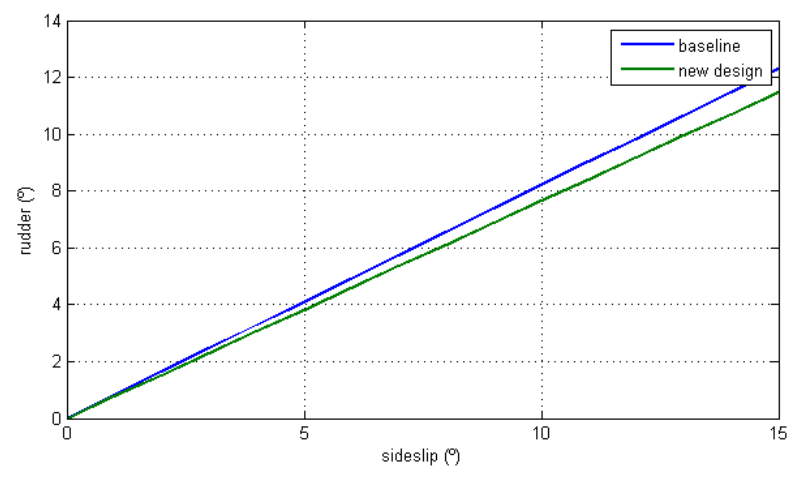

Figure 6.2: Effect of design changes on rudder position directional stability

Is it worth looking at Figure 6.2, which shows that required rudder deflection for maintaining steady sideslip has decreased.

It is important to restate that the solution presented is not unique, nor optimal. On the other hand, we do not consider the mechanical, or even economical, viability of introducing such changes. We just intend to exemplify how the information provided by parametric studies can be used. Although we fulfilled our design goal, it does not necessarily imply better flying qualities, because, contrary to what happens to piloted aircraft, rating criteria for UAV FQ is still not clearly defined yet. Nevertheless, some advantages/disadvantages of making the above changes have been identifyied. 


\section{Chapter 7}

\section{Conclusions and Future Work}

\subsection{Project Overview}

The objective of this project was to analyse how changes in aircraft design affected its flying qualities, which describe how well it performs the mission it was designed for. In order to do that, we used a Portuguese Air Force UAV as a model and predefined a set of eight design parameters to study, related to control surfaces and stabilizers size and position. As to the set of flying qualities, choice was not so straightforward.

Flying qualities depend on several parameters describing not only the aircraft dynamic behavior but also a pilot's effort to make it perform a certain task. Therefore, not all these parameters make sense to unmanned aircraft. We had to select those that were most representative of UAV performance.

The structure followed in this report reflects the way we conducted the project. It is mainly divided in two parts: one for background and other for the parametric studies:

\section{First Part}

Because we would need a dynamic model for the analysis, since flight tests weren't viable, we started by gathering information on how to derive a not too complex, but reliable, dynamic model for an aircraft. Research indicated a 6DOF nonlinear model, based on Newton secong Law, as adequate, but also that a linear decoupled model could give good results if the simulation envelope was centered in an equilibrium/trim point and not too wide. Therefore, Chapter 1 not only presents the 6DOF nonlinear model, but also a trim algorithm for four different flight conditions and another one for linearization. Neither of the algorithms are original.

Background study proceed with flying qualities theory. The goal was to understand which parameters best described aircraft effectiveness in fulfilling a certain task and that could be applicable to 
UAVS, at the same time. Based on that, we decided to study a set of static and dynamic stability characteristics. The next step was to find out how those parameters could be determined. Chapter 2 is entirely dedicated to this subject.

\section{Second Part}

Defined what to study and how to do it, we moved on to UAV analysis, starting by determining its 6DOF dynamic model. When computing model parameters, different methods were used in order to validate results. Those already available were recalculated. Then, we made a study on ANTEX current flying qualities, using not only the nonlinear and linearised model, but also a general linear decoupled model for an aircraft. This is the subject of chapter 4 .

Chapter 5 finally presents the parametric studies, the most important part of the project. We divided parametric studies in two parts: the first one consisted on understanding the dependence of flying qualities on model parameters and the second one on analyzing how changes in design affected model parameters. Both were based on sensitivity analysis. Then, using the concept of function composition we concluded on flying qualities dependence on specific design changes.

To illustrate how the above information could be used, we ended our project presenting a design example (see chapter 6). The objective was to improve certain flying qualities regarding a specific mission. We showed that improvement was possible by introducing slight design changes to the original configuration. The solution we present is not optimal, though. Optimization is outside the scope of this work.

\subsection{Conclusions}

Throughout the project, we have been able to draw important conclusions, which, together with the specific results obtained, represent our contribution to the vast area of flight dynamics and flying qualities. They are related to the following subjects:

1. Results validation

\section{Parametric Studies}

3. Applicability to other aircraft

When performing the different studies, we always sought to validate results, not only to reduce uncertainy, but also to check if we could use simpler methods for the different analysis, that, at the same time, provided more physical insight into the process. Through that we were able to conclude that, for the simulation envelope used and type of results required :

- DATCOM correlations for aerodynamic derivatives predict CFD results quite well. 
- The general linear decoupled model for an aircraft gives quite accurate results, except for two parameters: phugoid damping ratio and spiral stability.

Therefore, we used the above tools to perform the parametric studies. The two sensitivity analysis conducted in chapter 5 for that purpose revealed that:

- For the set of flying qualities under study, some model parameters are more important than others.

- Some model parameters don't have a significant effect on any of the flying qualites studied.

- It is possible to measure how uncertainty in model parameters affect flying qualities.

- Not all model parameters are sensitive to the selected design variables.

- Not all flying qualities are sensitive to the selected design variables

- If changes in design variables are boxed to \pm 20

Some of the above conclusions, which greatly simplify the study, couldn't be drawn if we didn't split the analysis in two.

The design example from Chapter 6 showed that it was possible to use information from parametric studies to make ANTEX more suitable to a specific mission, by introducing some design changes. However, the solution we present is not optimal nor accounts for mechanical viability of making such design changes. Both subjects are beyond the scope of this work.

Although we used a specific UAV to make our studies, they were conducted in a way such that some of the results could be potentially extrapolated to other aircraft. This was the original reason to perform parametric studies in two steps instead of just one, because the first one, which looks into the relation between flying qualties and model parameters, is not so dependent on the aircraft. We say "not so dependent" instead of independent because the baseline for parametric studies is the set of flying qualities of a specific aircraft.

However, tests made on two other aircraft, different in size,configuration and mission, validated previous results and so we can deduce that conclusions taken from the first part of parametric studies are indeed applicable to other aircraf configurations, at least to those having a conventional response.

\subsection{Future Work}

We managed to fulfill the initial objective of this project, which consisted on parameterizing the influence of some design parameters on UAV flying qualities. Moreover, we concluded that a part of the results was applicable to other aircraft (UAV or manned) configurations. Nevertheless, although the project has finished here, it raises some questions that require deeper studies, which need to be addressed to in the future. 
As it was mentioned in Section 1.1, it represents a contribution to a quite vast area, where there is still much to do. We took one of the possible direction that can be continued in several ways:

- Optimization

- Extension to other design parameters and flying qualities

- Inclusion in design stage

- UAV flying qualities rating

We presented a design example in last chapter whose goal was to improve an existing UAV, regarding a specific mission. But the solution presented is not optimal, and better characteristics can be achieved, considering both requirements and restrictions, if the results obtained in Chapter 5 are used together with and optimization tool.

Because of time limits, we had to restrict our study to a set of design parameters and flying qualities. As to the flying qualities, we tried to choose those most representative of an aircraft response aiming at a certain mission. Future works can extend this set - the same strategy followed here can be used.

The starting point of the project was an existing UAV, whose dynamics improvement by making some design modifications was to be analyzed. Therefore, we limited the analysis to design changes up to $20 \%$, which is still a quite large value. As a result, effects on FQ parameters were also limited to a certain magnitude. However, the knowlegde here acquired is also very useful for aircraft still being projected, where larger changes are allowed and, consequently, larger effects on flying qualities.

This project focuses on measuring certain FQ parameters, but once FQ requirements for UAVs are clearly defined, it will be possible to rate them based on the values of the different parameters here assessed.

Each of the four cases can use this project as a basis. Additionally, the various Matlab programs developed can be easily addapted to other aircraft and contribute to the creation of a more general tool for the type of studies here presented. 


\section{Appendix A}

\section{ANTEX-X03 Existing Data}

\section{A.1 Flight Envelope}

- Air Properties

\begin{tabular}{|c|c|c|c|c|c|}
\hline $\begin{array}{c}\mathrm{T} \\
(K)\end{array}$ & $\begin{array}{c}\text { Dens } \\
\left(\mathrm{kg} / \mathrm{m}^{3}\right)\end{array}$ & $\begin{array}{l}\text { Din Visc } \\
\left(N . s / m^{2}\right)\end{array}$ & $\begin{array}{l}\text { Kin Visc } \\
\left(m^{2} / s\right)\end{array}$ & $\begin{array}{c}\mathrm{Cp} \\
(k J / k g \cdot K)\end{array}$ & $\begin{array}{c}\mathrm{R} \\
(J / k g . K)\end{array}$ \\
\hline \multicolumn{6}{|c|}{ Ground: $0 f t$} \\
\hline 288.15 & 1.225 & $1.789 \times 10^{-5}$ & $1.460 \times 10^{-5}$ & 1.005 & 287 \\
\hline \multicolumn{6}{|c|}{ Cruise: ? } \\
\hline 288.15 & 1.225 & $1.789 \times 10^{-5}$ & $1.460 \times 10^{-5}$ & 1.005 & 287 \\
\hline \multicolumn{6}{|c|}{ Ceiling: $10000 \mathrm{ft}$} \\
\hline 268.34 & 0.90464 & $1.692 \times 10^{-5}$ & $1.870 \times 10^{-5}$ & 1.005 & 287 \\
\hline
\end{tabular}

- Flight Phases 


\begin{tabular}{cccc} 
Speed $(k m / h)$ & Alt & Mach & Reynolds \\
\hline \hline \multicolumn{4}{c}{ Take-off \& Landing } \\
50 & ground 0.0408 & $6.37 \times 10^{5}$ \\
& Cruise & \\
100 & cruise & $?$ \\
& & \\
& Max Speed & \\
130 & cruise & $?$ & $?$ \\
& ceiling 0.1101 & $? 1.29 \times 10^{6}$
\end{tabular}

The indicated values for take-off and landing speed correspond to the stall speed. The real ones haven't been accurately determined yet.

- Limits for Mach and Reynolds Numbers

\begin{tabular}{cc} 
Reynolds Number & Mach Number \\
\hline $\left.6.37 \times 10^{5} ; 1.29 \times 10^{6}\right]$ & {$[0.04 ; 0.1]$}
\end{tabular}

\section{A.2 Aerodynamic Characteristics}

\section{A.2.1 Assumptions}

- No coupling between longitudinal and lateral modes

- Aerodynamic coefficients expanded in Taylor Series - only first order terms considered

Both CFD and wind tunnel tests were performed, in cruise conditions.

For a valid comparison of results, the same model and flow properties should be used, or, if such isnt́ possible, dimensional and dynamic similarity should be guaranteed. Dynamic similarity implies matching of Reynolds, Froude and Mach number, as well as angle of attack. Only Reynolds number and angle of attack were accounted for, because:

- For rigid and fixed models Froude number isnt́ important

- For an incompressible regime $(M<0,3)$, as it is the case, the influence of Mach number can be neglected

Because of insufficient wind tunnel section dimensions, a 1:6 model had to be built. 


\section{A.2.2 Summary of Relevant Flight Conditions}

\begin{tabular}{r|ll} 
Speed & $100 \mathrm{~km} / \mathrm{h}$ & \\
Kinematic Viscosity & $1.46 \times 10^{-5} \mathrm{~m}^{2} / \mathrm{s}$ & $\begin{array}{l}\text { MSL properties used because cruise al- } \\
\text { titude isn't known }\end{array}$ \\
Reynolds & $1.3 \times 10^{6}$ & Considering the tip chord $(0.67 \mathrm{~m})$
\end{tabular}

For Reynolds equivalence, a speed of $166.8 \mathrm{~m} / \mathrm{s}$ was required. Due to tunnel limitations, whose maximum speed is $70 \mathrm{~m} / \mathrm{s}$, a speed of $50 \mathrm{~m} / \mathrm{s}(180 \mathrm{~km} / \mathrm{h})$ was used, corresponding to a Reynolds of $4 \times 10^{5}$.

\section{A.2.3 Tests Performed}

- Through both CFD software and Wind Tunnel

\begin{tabular}{ccccc} 
Parameter & Min & Max & Step & Observations \\
\hline \hline$\alpha\left(^{\circ}\right)$ & -12 & 18 & 2 & $\alpha=0$ : flow aligned with wing root chord \\
$\beta\left(^{\circ}\right)$ & -10 & 10 & 2 & \\
$\delta e\left(^{\circ}\right)$ & -20 & 20 & & Tested at 5 different $\alpha(\mathrm{WT})$ and at $\alpha=0$ \\
$\delta r\left(^{\circ}\right)$ & 0 & 20 & 5 & $\begin{array}{l}\text { Tested at } 5 \text { different } \alpha(\mathrm{WT}) \text { and at } \alpha=0 \\
\text { Symmetry check at }-10^{\circ}\end{array}$
\end{tabular}

- Trough Wind Tunnel only

\begin{tabular}{ccccl} 
Parameter & Min & Max & Step & Observations \\
\hline \hline$\delta a\left(^{\circ}\right)$ & 0 & 20 & 5 & $\begin{array}{l}\text { Tested at } 5 \text { different } \alpha(\mathrm{WT}) \text { and at } \alpha=0 \\
\text { Symmetry check at }-10^{\circ}\end{array}$ \\
$\delta f\left(^{\circ}\right)$ & 0 & 20 & 10 & Tested at 5 different $\alpha$
\end{tabular}

- Through CFD software only 


\begin{tabular}{ccccl} 
Parameter & Min & Max & Step & Observations \\
\hline \hline $\mathrm{p}(\% / s)$ & -30 & - & 10 & $\begin{array}{l}\text { Mid Values: }-5 \text { and }-15 \\
\text { Tested at } \alpha=0\end{array}$ \\
$\mathrm{q}(\% / s)$ & -5 & 10 & 5 & Tested at $\alpha=0$ \\
$\mathrm{r}(\% / s)$ & -20 & 20 & 5 & Tested at $\alpha=0$
\end{tabular}

\section{A.2.4 Additional Remarks}

\section{Wind Tunnel}

- Open working section

- The block holding the model, and which size was comparable to the fuselage, may have caused significant interference on the flow and consequently on the measured parameters

- Balance needed calibration

- Results dont́ seem accurate and should be considered as merely representative

\section{CFD}

- Software: CFX, which solves the Navier-Stokes equations

- Turbulence model: Shear Stress Transport

- Lift coefficient was validated through Lifting Line Theory

- Convergence criteria based on residual value of pressure, continuity and turbulence equations; a value of $10^{-4}$ was used

- Unable to detect stall

- Problem didnt́ converge for angles of attack beyond $18^{\circ}$

\section{A.2.4.1 Wind Tunnel and CFD Results Comparison}

Though not shown here, there are discrepancies between the two methods and therefore it might be important to validate some results. Qualitatively, the results go as expected, but attention should be paid to the non positive value of $C_{m 0}$ in the Cm vs. $\alpha$ curve, for a zero deflection of the elevator. 


\section{A.2.4.2 Stability Derivatives}

Because of the wind tunnel inaccuracies already mentioned, CFD results are presented whenever possible (values in bold are used for those obtained through the wind tunnel tests). ${ }^{1}$

- Longitudinal (control surface derivatives for zero angle of attack)

\begin{tabular}{c|cccccc|}
\cline { 2 - 6 } \multicolumn{1}{c}{} & $u$ & $\alpha$ & $q$ & $\dot{\alpha}$ & $\delta e$ & $\delta f$ \\
\cline { 2 - 7 } & $C_{L}$ & 5.197 & 1.086 & -0.3839 & $\mathbf{0 . 7 0 5 9}$ \\
$C_{D}$ & & nonconstant & 0 & nonconstant & $\mathbf{0 . 0 2 4 6 4}$ \\
$C_{m}$ & -0.5844 & -0.267 & 0.911 & $\mathbf{0 . 0 8 5 3 7}$ \\
\hline
\end{tabular}

- Lateral (results for zero angle of attack)

\begin{tabular}{|c|cccccc|}
\cline { 2 - 6 } \multicolumn{1}{c|}{} & $\beta$ & $p$ & $r$ & $\dot{\beta}$ & $\delta r$ & $\delta a$ \\
\hline$C_{L}$ & -0.4034 & -0.008 & 0.1576 & -0.1261 & $\mathbf{0 . 0 2 4 6 4}$ \\
$C_{D}$ & 0.04354 & 0.00057 & -0.0235 & 0.04011 & $\mathbf{0 . 1 3 1 2}$ \\
$C_{m}$ & -0.043 & -0.0579 & 0.01776 & -0.0046 & $\mathbf{0}$ \\
\hline
\end{tabular}

\section{A.3 Center of Gravity (CG) Position an Inertia Tensor}

It was found experimentally that the CG lies in the plane of symmetry of the UAV (longitudinal plane), $0.555 \mathrm{~m}$ above the ground and $0.9 \mathrm{~m}$ aft of the nose landing gear. Because of mirror symmetry of ANTEX, its inertia tensor is of the form:

$$
I=\left[\begin{array}{rrr}
I_{x x} & -I_{x y} & -I_{x z} \\
-I_{x y} & I_{y y} & -I_{y z} \\
-I_{x z} & -I_{y z} & I_{z z}
\end{array}\right]
$$

Experiments were made in order to calculate it, but validation failed. So, this information is not yet available. In the control system thesis, as this data was needed, approximations were made through comparison with similar aircraft, namely Aerosonde. The following values $\left(\mathrm{kg} \cdot \mathrm{m}^{2}\right.$ units $)$ were used:

$$
I=\left[\begin{array}{rrr}
182.2 & 0 & -23 \\
0 & 250.9 & 0 \\
-23 & 0 & 389.06
\end{array}\right]
$$

\section{A.4 Open Loop Stability}

Both linear and nonlinear models were used to describe the UAV flight dynamics, based on the data presented previously and actuators, landing gear and engine characteristics (the three last have

\footnotetext{
${ }^{1}$ Angle unit is radian
} 
been estimated). Although there is a good agreement between the linear and non-linear models, openloop analysis revealed some unexpected results, in the sense that they contradict experimental data: unstable phugoid and dutch roll modes. The aerodynamic, engine, inertia and landing gear data might be a source of the problem.

\section{A.5 Existing Files}

- Computational geometric model (IGES and PDF files)

- CFD results (EXCEL file)

- Mesh files (according to the authors of the CFD analysis, the mesh should be improved)

- Master Thesis:

- Félix and Saraiva [9]

- Coutinho [6] 


\section{Appendix B}

\section{Methods for Estimating Aerodynamic Derivatives}

This appendix presents DATCOM methods to estimate aerodynamic derivatives, but only those whose determination is not too complex. Expressions below have already been particularised for UAV ANTEX-M X03. Some parameters are also defined.

\section{B.1 Sign Convention}

The sign convention used for aerodynamic forces and angles is as presented on Figure B.1. Rolling moment $L$ (do not confuse with lift force), which is not represented there, is positive if directed along the positive $x$ axis (left wing up). 


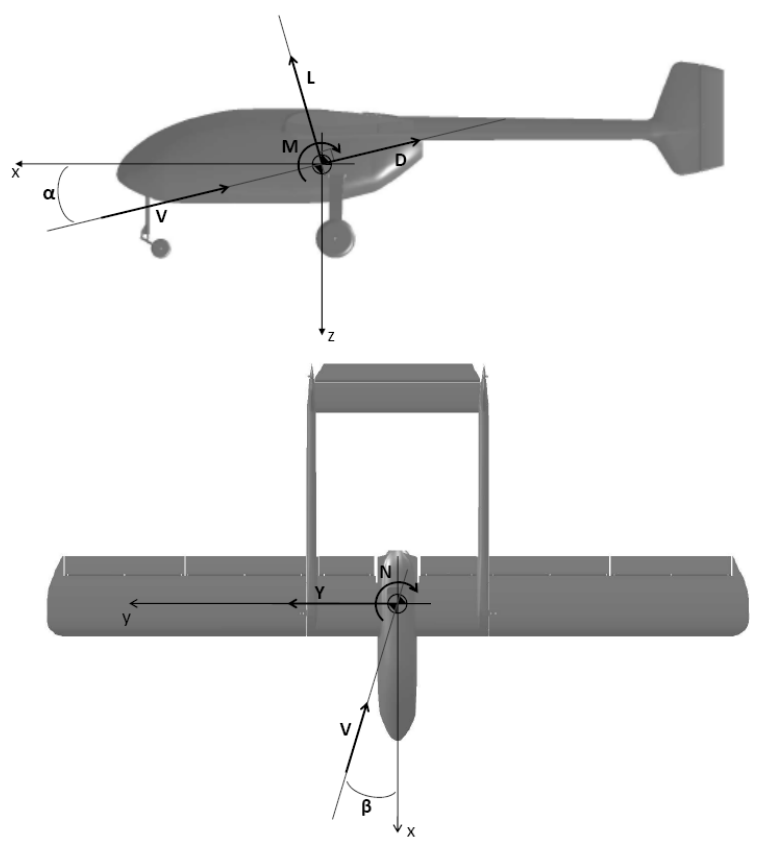

Figure B.1: Sign convention for aerodynamic forces and angles

\section{B.2 Aircraft Controls}

Typical aircraft control surfaces are: elevator, rudder and ailerons. Figure B.2 shows where these are located on ANTEX-M X03.

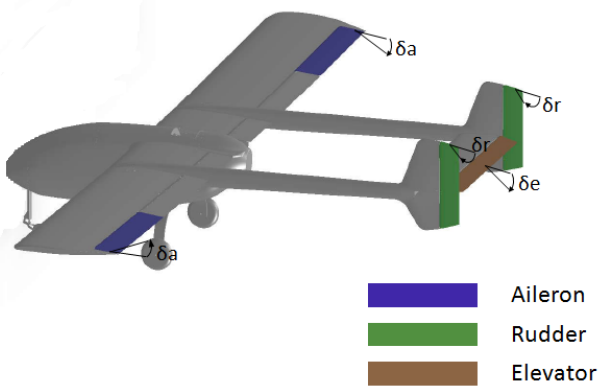

Figure B.2: Control Surfaces

\section{B.3 Geometric Parameters Definition}

Figure B.3 defines geometric parameters appearing on empiric expressions. Baseline values for these parameters are: 


\begin{tabular}{|c|c|}
\hline Wing & Aileron \\
\hline $\begin{aligned} c & =0.67 m \\
b & =5.7 m \\
S & =b c \\
A & =\frac{b}{c}\end{aligned}$ & $\begin{aligned} c_{a i l} & =0.18 m \\
b_{a i l} & =0.95 m \\
\eta_{i} & =0.03\end{aligned}$ \\
\hline Vertical Stabilizer + Rudder & Horizontal Stabilizer + Elevator \\
\hline $\begin{aligned} c_{v} & =0.3 \mathrm{~m} \\
b_{v} & =0.55 \mathrm{~m} \\
S_{v} & =b_{v} c_{v} \\
A_{v} & =\frac{b_{v}}{c_{v}} \\
t_{v} & =0.03 \mathrm{~m} \\
c_{r u d} & =0.11 \mathrm{~m}\end{aligned}$ & $\begin{aligned} c_{h} & =0.35 \mathrm{~m} \\
b_{h} & =1.35 \mathrm{~m} \\
S_{h} & =b_{h} c_{h} \\
A_{h} & =\frac{b_{h}}{c_{h}} \\
t_{h} & =0.03 \mathrm{~m} \\
c_{e l} & =0.13 \mathrm{~m}\end{aligned}$ \\
\hline \multicolumn{2}{|c|}{ Other Parameters } \\
\hline $\begin{array}{l}l_{h} \\
l_{v} \\
z_{v}\end{array}$ & $\begin{array}{l}1.74 m \\
1.75 m \\
0.32 m\end{array}$ \\
\hline
\end{tabular}

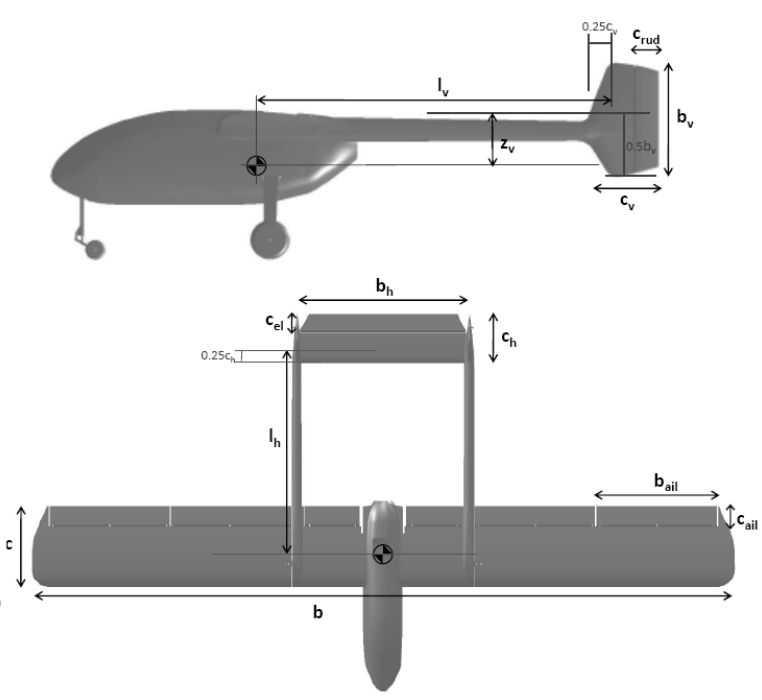

Figure B.3: Geometric Parameters 


\section{B.4 Longitudinal Derivatives}

\section{Stability Derivatives}

- Angle of Attack Derivatives: $C_{L_{\alpha}}, C_{m_{\alpha}}$

$$
C_{L_{\alpha}} \simeq C_{L_{\alpha_{w}}}+C_{L_{\alpha_{h t a i l}}}
$$

Wing Contribution:

$$
C_{L_{\alpha_{w}}}=\frac{2 \pi A}{2+\sqrt{A^{2}+4}}
$$

Tail Contribution:

$$
C_{L_{\alpha_{h t a i l}}}=C_{L_{\alpha_{h}}} \frac{S_{h}}{S}\left(1-\frac{\partial \epsilon}{\partial \alpha}\right)
$$

where

$$
\begin{gathered}
C_{L_{\alpha_{h}}}=\frac{2 \pi A_{h}}{2+\sqrt{A_{h}^{2}+4}} \\
\frac{\partial \epsilon}{\partial \alpha}=4.44\left(K_{A} K_{\lambda}\right)^{1.19}\left(\frac{1-\frac{h_{h}}{b}}{\sqrt[3]{\frac{2 l_{h}}{b}}}\right)^{1.19} \\
C_{m_{\alpha}}=C_{L_{\alpha_{w b}}}\left(h-h_{n_{w b}}\right)-C_{L_{\alpha_{h}}} \frac{l_{h} S_{h}}{\bar{c} S}\left(1-\frac{\partial \epsilon}{\partial \alpha}\right)
\end{gathered}
$$

- $q$ Derivatives: $C_{L_{q}}, C_{m_{q}}$

$$
C_{L_{q}}=C_{L_{q_{w b}}}+C_{L_{q_{h t a i l}}}
$$

Tail Contribution:

$$
\begin{gathered}
C_{L_{q_{\text {tail }}}}=2 C_{L_{\alpha_{h}}} \frac{S_{h} l_{h}}{S c} \\
C_{m_{q}} \simeq C_{m_{q_{h t a i l}}}=-2 C_{L_{\alpha_{h}}} \frac{S_{h} l_{h}^{2}}{S c^{2}}
\end{gathered}
$$


- Angle of Attack Rate Derivatives $C_{L_{\dot{\alpha}}}, C_{m_{\dot{\alpha}}}$

$$
C_{L_{\dot{\alpha}}}=C_{L_{\dot{\alpha}_{w b}}}+C_{L \dot{\alpha}_{q_{h t a i l}}}
$$

Tail Contribution:

$$
\begin{gathered}
C_{L \dot{\alpha}_{q_{h t a i l}}}=2 C_{L_{\alpha_{h}}} \frac{S_{h} l_{h}}{S c} \frac{\partial \epsilon}{\partial \alpha} \\
C_{m_{\dot{\alpha}}} \simeq C_{m_{\dot{\alpha}_{h t a i l}}}=-2 C_{L_{\alpha_{h}}} \frac{S_{h} l_{h}^{2}}{S c^{2}} \frac{\partial \epsilon}{\partial \alpha}
\end{gathered}
$$

\section{Control Derivatives}

- Elevator Derivatives: $C_{L_{\delta_{e}}}, C_{m_{\delta_{e}}}$

$$
C_{L_{\delta_{e}}}=C_{l_{\delta_{e}}} \frac{C_{L_{\alpha_{h}}}}{2 \pi\left(1+\left(\frac{t_{h}}{c_{h}}\right)\right)} \frac{\left(a_{\delta}\right)_{C_{L}}}{\left(a_{\delta}\right)_{C_{l}}} \frac{S_{h}}{S}
$$

where

$$
\begin{aligned}
& C_{l_{\delta e}}=\frac{C_{l_{\delta_{e}}}}{C_{l_{\delta_{e_{\text {theory }}}}}}\left(17.6\left(\frac{c_{e l}}{c_{h}}\right)^{3}-25.2\left(\frac{c_{e l}}{c_{h}}\right)^{2}+17.3 \frac{c_{e l}}{c_{h}}+1\right) \frac{S_{h}}{S}
\end{aligned}
$$

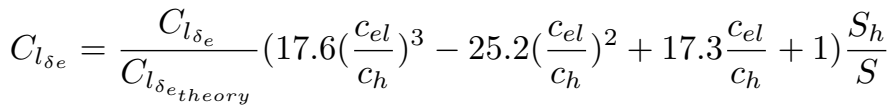

$$
\begin{aligned}
& \frac{\left(a_{\delta}\right)_{C_{L}}}{\left(a_{\delta}\right)_{C_{l}}} \simeq 1.055 \\
& C_{m_{\delta_{e}}}=-C_{L_{\delta_{e}}} \frac{l_{h}}{\bar{c}}
\end{aligned}
$$

Though not a derivative, we also need another aerodynamic parameter, $C_{m_{0}}$, particularly tail contribution.

$$
C_{m_{0_{h t a i l}}}=-C_{L_{\alpha_{h}}} \frac{S_{h} l_{h}}{S c}\left(i_{t}+\epsilon_{0}\right)
$$

where $i_{t}$ is the horizontal tail incidence relative to zero angle of attack line and $\epsilon_{0}$ is the downwash angle for zero angle of attack (we assume that $\left(i_{t}+\epsilon_{0}\right)=-1^{\circ}$ ). 


\section{B.5 Lateral Derivatives}

\section{Stability Derivatives}

- Sideslip Derivatives: $C_{Y_{\beta}}, C_{l_{\beta}}, C_{n_{\beta}}$

$$
C_{Y_{\beta}}=C_{Y_{\beta_{w}}}+C_{Y_{\beta_{b}}}+C_{Y_{\beta_{v t a i l}}}
$$

Tail Contribution:

$$
C_{Y_{\beta_{v t a i l}}}=-1.9 C_{Y_{\beta_{v_{e f f}}}} \frac{S_{v}}{S}
$$

where

$$
\begin{gathered}
C_{Y_{\beta_{v_{e f f}}}=-0.0071 A_{v}^{4}+0.1001 A_{v}^{3}-0.6303 A_{v}^{2}+2.3477 A_{v}-0.0444} \\
C_{l_{\beta}} \simeq C_{l_{\beta_{w b}}}+C_{l_{\beta_{v t a i l}}}
\end{gathered}
$$

Tail Contribution:

$$
\begin{gathered}
C_{l_{\beta_{v t a i l}}}=C_{Y_{\beta_{v t a i l}}} \frac{z_{v}}{b} \\
C_{n_{\beta}} \simeq C_{n_{\beta_{b}}}+C_{n_{\beta_{v t a i l}}}
\end{gathered}
$$

Tail Contribution:

$$
C_{n_{\beta_{v t a i l}}}=-C_{Y_{\beta_{v t a i l}}} \frac{l_{v}}{b}
$$

- $p$ Derivatives: $C_{Y_{p}}, C_{l_{p}}, C_{n_{p}}$

$$
\begin{gathered}
C_{Y_{p}} \simeq C_{Y_{p_{v t a i l}}}=\frac{2 z_{v}}{b} C_{Y_{\beta_{v t a i l}}} \\
C_{l_{p}}=C_{l_{p_{w b}}}+C_{l_{p_{h t a i l}}}+C_{l_{p_{v t a i l}}}
\end{gathered}
$$

Tail Contribution: 


$$
\begin{gathered}
C_{l_{p_{h t a i l}}}=\left[0.0076 \frac{A_{h}^{2}}{1+\frac{t_{h}}{c_{h}}}-0.167 A_{h}-0.479\left(1+\frac{t_{h}}{c_{h}}\right)\right] \frac{S_{h}}{S}\left(\frac{b_{h}}{b}\right)^{2} \\
C_{l_{p_{v t a i l}}}=2\left(\frac{z_{v}}{b}\right)^{2} C_{Y_{\beta_{v t a i l}}} \\
C_{n_{p}}=C_{n_{p_{w}}}+C_{n_{p_{v t a i l}}}
\end{gathered}
$$

Tail Contribution:

$$
C_{n_{p_{v t a i l}}}=-\frac{2}{b^{2}} l_{v} z_{v} C_{Y_{\beta_{v t a i l}}}
$$

- $r$ Derivatives: $C_{Y_{r}}, C_{l_{r}}, C_{n_{r}}$

$$
\begin{gathered}
C_{Y_{r}} \simeq C_{Y_{r_{v t a i l}}}=-\frac{2}{b} l_{v} C_{Y_{\beta_{v t a i l}}} \\
C_{l_{r}}=C_{l_{r_{w}}}+C_{l_{r_{v t a i l}}}
\end{gathered}
$$

Tail Contribution:

$$
\begin{gathered}
C_{l_{r_{v t a i l}}}=-\frac{2}{b^{2}} l_{v} z_{v} C_{Y_{\beta_{v t a i l}}} \\
C_{n_{r}}=C_{n_{r_{w}}}+C_{n_{r_{v t a i l}}}
\end{gathered}
$$

Tail Contribution:

$$
C_{n_{r_{v t a i l}}}=\frac{2}{b^{2}} l_{v}^{2} C_{Y_{\beta_{v t a i l}}}
$$

\section{B.5.1 Control Derivatives}

- Aileron Derivatives: $C_{Y_{\delta_{a}}}, C_{l_{\delta_{a}}}, C_{n_{\delta_{a}}}$

$$
\begin{gathered}
C_{Y_{\delta_{a}}} \simeq 0 \\
C_{l_{\delta_{a}}}=0.2523 C_{l_{\delta_{\text {theory }}}}\left(\frac{\beta C_{l_{\delta}}^{\prime}}{k}\right)
\end{gathered}
$$

where

$$
\begin{aligned}
\left(\frac{\beta C_{l_{\delta}}^{\prime}}{k}\right) & =0.843+0.8887 \eta_{i}^{3}-1.9018 \eta_{i}^{2}+0.1589 \eta_{i} \\
C_{l_{\delta_{\text {theory }}}} & =25.253\left(\frac{c_{\text {ail }}}{c}\right)^{3}-31.212\left(\frac{c_{\text {ail }}}{c}\right)^{3}+18.846\left(\frac{c_{\text {ail }}}{c}\right)+0.9317
\end{aligned}
$$




$$
C_{n_{\delta_{a}}}=0.09 K C_{l_{\delta_{a}}}
$$

where

$$
K=-0.1324 \eta_{i}^{3}+0.0686 \eta_{i}^{2}-0.0425 \eta_{i}-0.1571
$$

- Rudder Derivatives: $C_{Y_{\delta_{r}}}, C_{n_{\delta_{r}}}, C_{n_{\delta_{r}}}$

$$
\left.C_{Y_{\delta_{r}}}=-2.17 C_{L_{\alpha_{v}}} \alpha_{\delta_{C_{l}}}\right) \frac{S_{v}}{S}
$$

where

$$
\begin{gathered}
C_{L_{\alpha_{v}}}=\frac{2 \pi\left(1.03 A_{v}\right)}{2+\sqrt{\left(1.03 A_{v}\right)^{2}+4}} \\
\alpha_{\delta_{C_{l}}}=3.125\left(\frac{c_{r u d}}{c_{v}}\right)^{4}-8.1944\left(\frac{c_{r u d}}{c_{v}}\right)^{3}+8.1042\left(\frac{c_{r u d}}{c_{v}}\right)^{2}-4.0323\left(\frac{c_{r u d}}{c_{v}}\right)-0.0012 \\
C_{l_{\delta_{r}}}=C_{Y_{\delta_{r}}} \frac{z_{v}}{b} \\
C_{n_{\delta_{r}}}=-C_{Y_{\delta_{r}}} \frac{l_{v}}{b}
\end{gathered}
$$

\section{B.6 Dimensional Derivatives}

Equations (2.34) and (2.35) make use of dimensional derivatives. They can be determined from the following expressions: 


\section{Longitudinal}

$$
\begin{aligned}
X_{u} & =-\frac{\bar{q} S}{V}\left(C_{D_{u}}+2 C_{D_{t r i m}}-C_{T_{u}}-2 C_{T_{t r i m}}\right) \\
Z_{u} & =-\frac{\bar{q} S}{V}\left(C_{L_{u}}+2 C_{L_{t r i m}}\right) \\
M_{u} & =\frac{\bar{q} S c C_{m_{u}}}{V} \\
X_{w} & =-\frac{\bar{q} S}{V}\left(C_{D_{\alpha}}-C_{L_{t r i m}}\right) \\
Z_{w} & =-\frac{\bar{q} S}{V}\left(C_{L_{\alpha}}+C_{L_{t r i m}}\right) \\
M_{w} & =\frac{\bar{q} S c C_{m_{\alpha}}}{V} \\
Z_{q} & =\frac{\bar{q} S c C_{L_{q}}}{2 V} \\
M_{q} & =\frac{\bar{q} S c^{2} C_{m_{q}}}{2 V} \\
Z_{\dot{w}} & =\frac{\bar{q} S c C_{L_{\dot{\alpha}}}}{2 V^{2}} \\
M_{\dot{w}} & =\frac{\bar{q} S c C_{m_{\dot{\alpha}}}}{2 V^{2}}
\end{aligned}
$$

where

$$
\begin{array}{r}
C_{T_{\text {trim }}}=C_{D_{\text {trim }}}+\frac{m g}{\bar{q} S} \sin \theta_{\text {trim }} \\
C_{T_{u}}=-3 C_{T_{\text {trim }}}
\end{array}
$$

\section{Lateral}

$$
\begin{aligned}
Y_{v} & =\frac{\bar{q} S C_{Y_{\beta}}}{V} \\
L_{v} & =\frac{\bar{q} S b C_{l_{\beta}}}{V} \\
N_{v} & =\frac{\bar{q} S b C_{n_{\beta}}}{V} \\
Y_{p} & =\frac{\bar{q} S b C_{Y_{p}}}{2 V} \\
L_{p} & =\frac{\bar{q} S b^{2} C_{l_{p}}}{2 V} \\
N_{p} & =\frac{\bar{q} S b^{2} C_{n_{p}}}{2 V} \\
Y_{r} & =\frac{\bar{q} S b C_{Y_{r}}}{2 V} \\
L_{r} & =\frac{\bar{q} S b^{2} C_{l_{r}}}{2 V} \\
N_{r} & =\frac{\bar{q} S b^{2} C_{n_{r}}}{2 V}
\end{aligned}
$$

Besides derivatives, there are also some inertia terms on equation (2.35) to define: 


$$
\begin{aligned}
I_{x x}^{\prime} & =\frac{I_{x x} I_{z z}-I_{x z}^{2}}{I_{z z}} \\
I_{z z}^{\prime} & =\frac{I_{x x} I_{z z}-I_{x z}^{2}}{I_{z z}} \\
I_{x x}^{\prime} & =\frac{I_{x z}}{I_{x x} I_{z z}-I_{x z}^{2}}
\end{aligned}
$$




\section{Appendix C}

\section{First and Second Order Systems}

This appendix presents methods to determine some characteristics of first and second order systems responses.

\section{C.1 First Order Systems}

The general form of a first order system is:

$$
\frac{k}{s+a}
$$

where $k$ is a constant and $a$ is the pole/eigenvalue of the system.

The most important parameter describing its dynamics is time constant $\tau$, which is an indicator of how quick the system responds to an input. If considering a step input, $\tau$ is time the system takesto reach 63.2 of its final steady-state value, after a step input. It is given by:

$$
\tau=\frac{1}{a}
$$

It can also be measured experimentally, as suggested on Figure C.1. The term ss in the $y$ axis means steady-state value.

\section{C.2 Second Order System}

The general form of a second order system is:

$$
\frac{k}{s^{2}+2 \xi \omega_{n} s+\omega_{n}^{2}}
$$

where $k$ is a constant, $\xi$ the damping ration and $\omega_{n}$ the undamped/natural frequency of the system. If $0<\xi<1$, the system has a pair of complex conjugate poles $z_{1}$ and $z_{2}$ given by: 


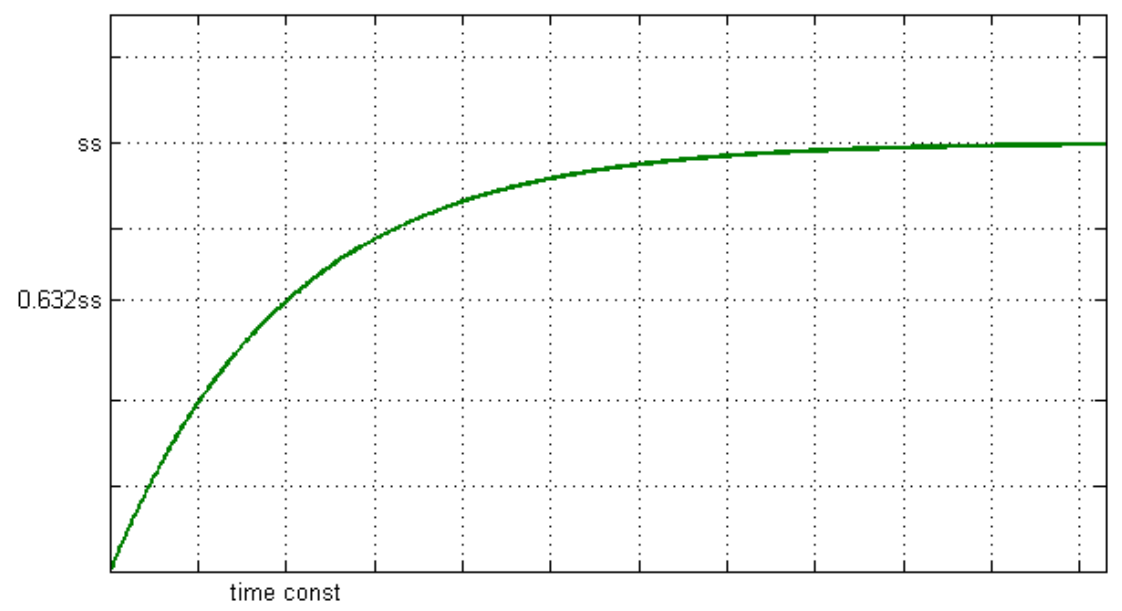

Figure C.1: Geometric Parameters

$$
z_{1,2}=-\xi \omega_{n} \pm \omega_{n} \sqrt{1-\xi^{2}}
$$

It is also possible to measure $\xi$ and $\omega_{n}$ experimentally through a method called logarithmic decrement:

Consider Figure XX, which shows the typical response of a second order system, in this case to a step input. Because oscillations eventually subside, the system is said to be dynamically stable.

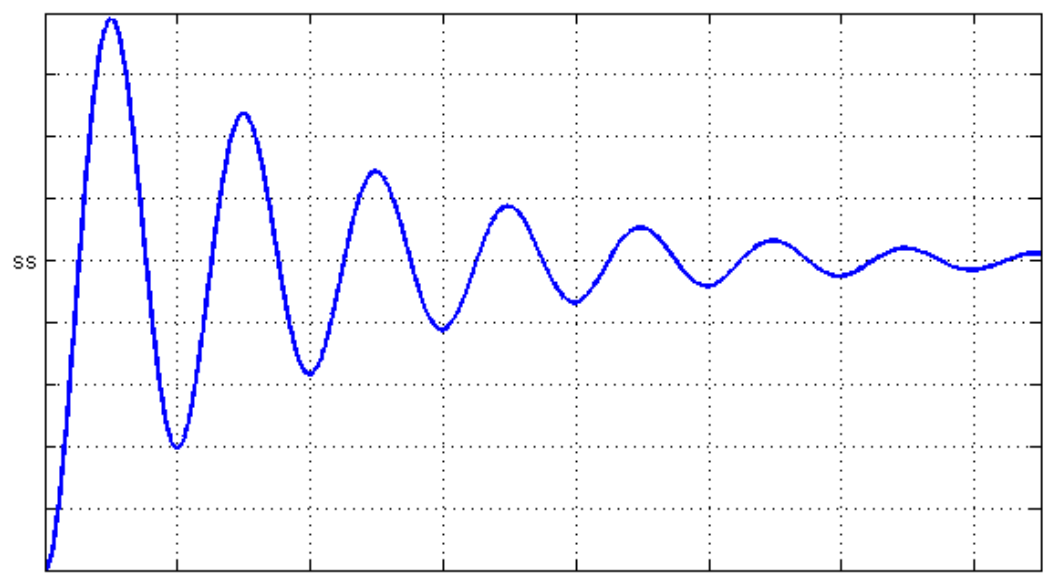

Figure C.2: Geometric Parameters

The logarithmic decrement of oscillations is given by the following ratio:

$$
\delta=\frac{1}{m} \ln \frac{x_{n}}{x_{n+m}}
$$


where $x_{n}$ is the amplitude of peak $n$ and $x_{n+m}$ the amplitude of a peak $m$ cycles away from peak $n$. These amplitude values are relative to the steady-state value, not to the initial condition.

The damping ration of the system can be found by:

$$
\xi=\frac{\delta}{\sqrt{4 \pi^{2}+\delta^{2}}}
$$

and the undamped frequency by

$$
\omega_{n}=\frac{2 \pi m}{\left(T_{n+m}-T_{n}\right) \sqrt{1-\xi^{2}}}
$$

where $T_{n}$ and $T_{n+m}$ are the times at which peaks $n$ and $n+m$ occur, respectively.

Another parameter that is worth analisyng, both for first and second order responses is time to double/half amplitude, $T_{2}$. Time to half is related to stable/convergent systems and time to double to unstable/divergent systems.

Time to double/half is can be determined by the following expression:

$$
T_{2}=\frac{0.6931}{|n|}
$$

where $n$ is the real part of the system poles. 


\section{Appendix D}

\section{Moments and Products of Inertia of}

\section{Regular Volumes}

This appendix presents expressions for moments and products of inertia of some ANTEX-M X03 components.

In order to determine the the contribution of some components to ANTEX inertia matrix, we model it with regular volumes such as parallelipepeds and cylinders, as shown in Figure D.1. According to it, contributions from horizontal tail plus elevator, vertical tails plus rudders and tail booms are as listed on Table D.2.
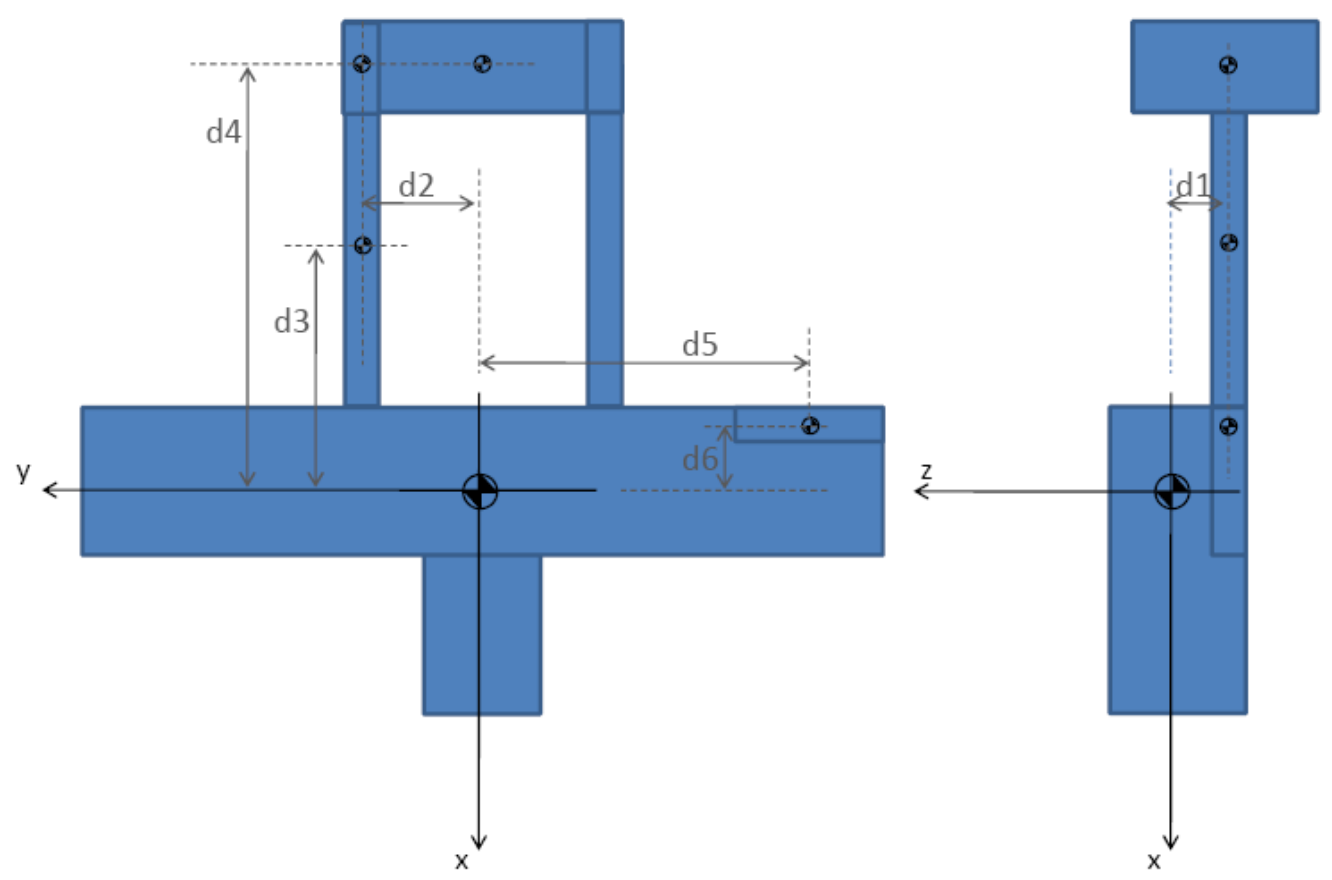

Figure D.1: ANTEX model 
Baseline values for parameters on Figure D.2 and Table D.2 are:

\begin{tabular}{|c|c|}
\hline Boom & Aileron \\
\hline $\begin{aligned} L & =1.23 \mathrm{~m} \\
r & =0.09 \mathrm{~m} \\
m & =1.59 \mathrm{~kg}\end{aligned}$ & $\begin{aligned} b & =0.95 m \\
w & =0.18 m \\
h & =0.03 \\
m & =0.38 \mathrm{~kg}\end{aligned}$ \\
\hline Vertical Stabilizer + Rudder & Horizontal Stabilizer + Elevator \\
\hline $\begin{aligned} b & =0.55 m \\
w & =0.3 m \\
h & =0.03 \\
m & =0.58 \mathrm{~kg}\end{aligned}$ & $\begin{array}{l}b=1.35 m \\
w=0.35 m \\
h=0.03 \\
m=1.42 \mathrm{~kg}\end{array}$ \\
\hline \multicolumn{2}{|c|}{ Other Parameters } \\
\hline $\begin{array}{l}a_{1} \\
d_{2} \\
d_{3} \\
d_{4} \\
d_{5} \\
d_{6}\end{array}$ & $\begin{array}{l}0.26 m \\
0.70 m \\
0.84 m \\
1.83 m \\
2.24 m \\
0.3 m\end{array}$ \\
\hline
\end{tabular}




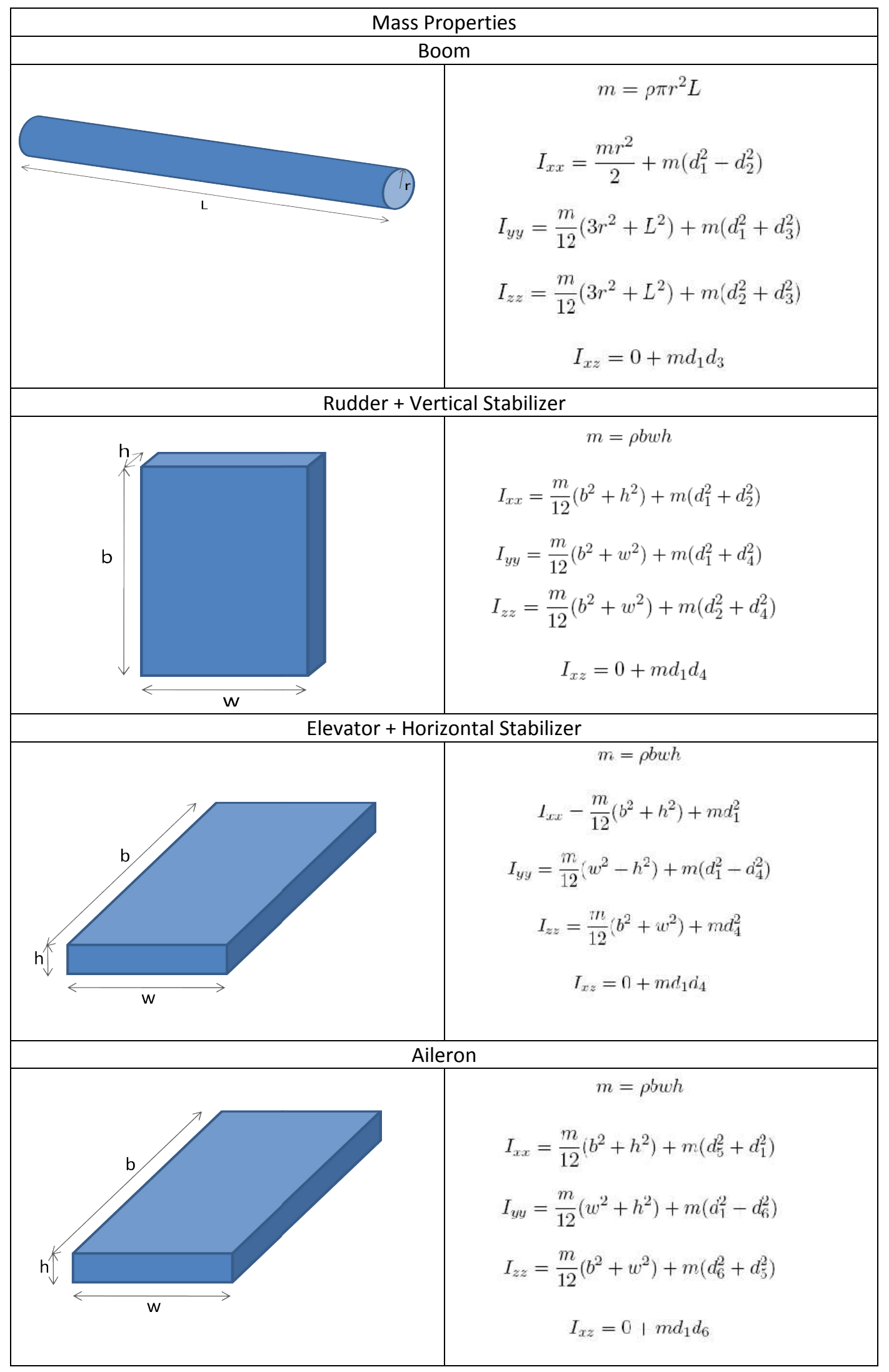

Figure D.2: Approximation of aircraft parts as standard geometric figures 


\section{Appendix E}

\section{Sensitivity Analysis for Different}

\section{Aircraft}

We study flying qualities sensitivity to model parameters using two different baseline configurations: Aircraft 1 and Aircraft 2 (see Section 5.5. Figures E.1 to E.8 show results for dynamic characteristics.

\section{E.1 Longitudinal Flying Qualities}

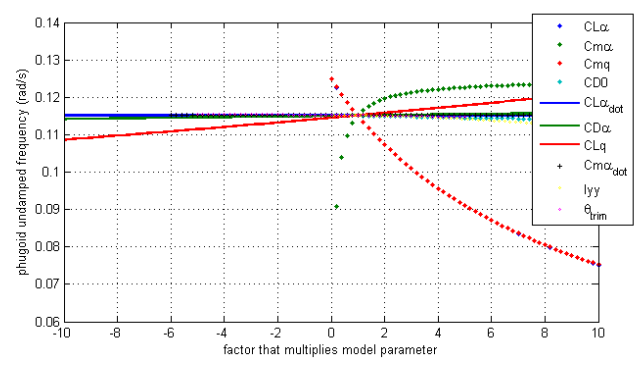

(a) Aircraft 1

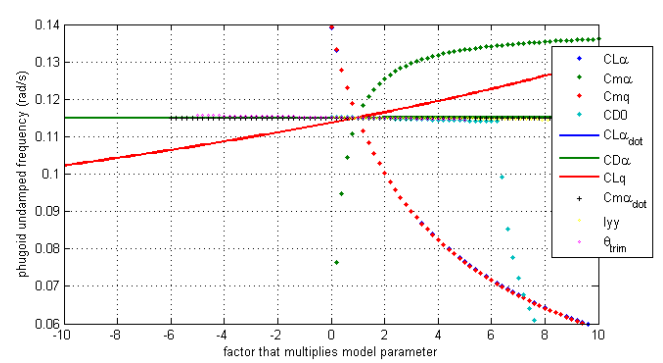

(b) Aircraft 2

Figure E.1: Phugoid undamped frequency

\section{E.2 Lateral Flying Qualities}




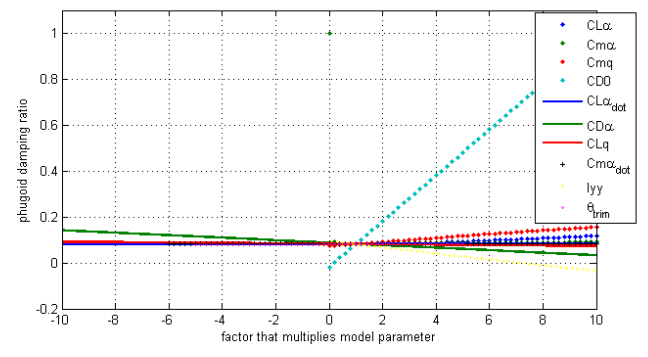

(a) Aircraft 1

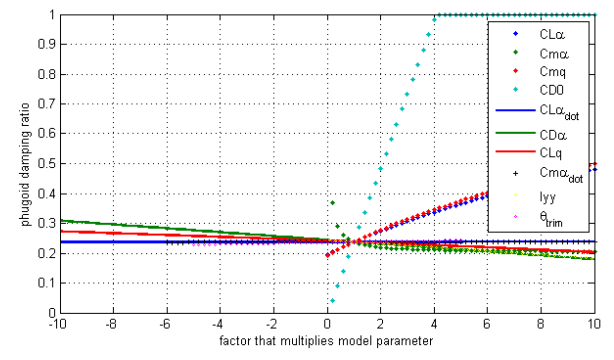

(b) Aircraft 2

Figure E.2: Phugoid damping ratio

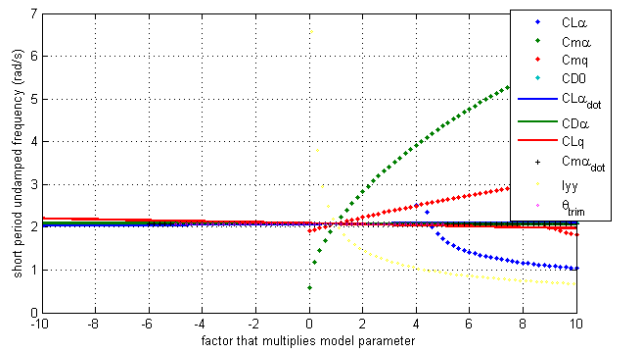

(a) Aircraft 1

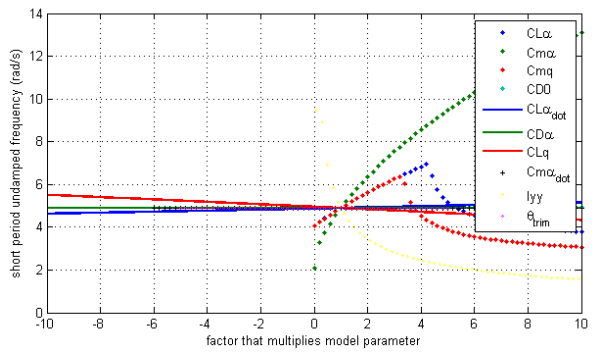

(b) Aircraft 2

Figure E.3: Short period undamped frequency

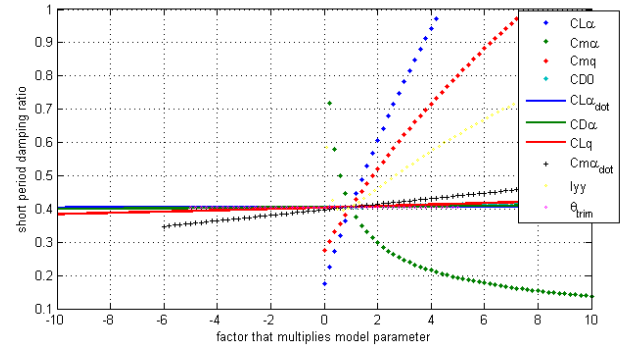

(a) Aircraft 1

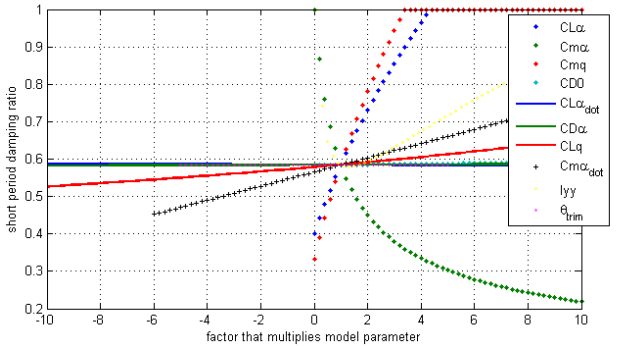

(b) Aircraft 2

Figure E.4: Short period damping ratio 


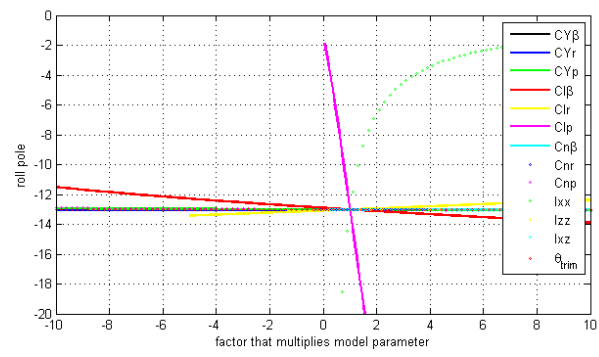

(a) Aircraft 1

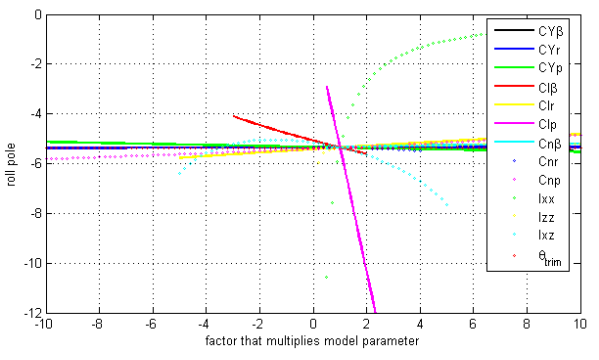

(b) Aircraft 2

Figure E.5: Roll pole

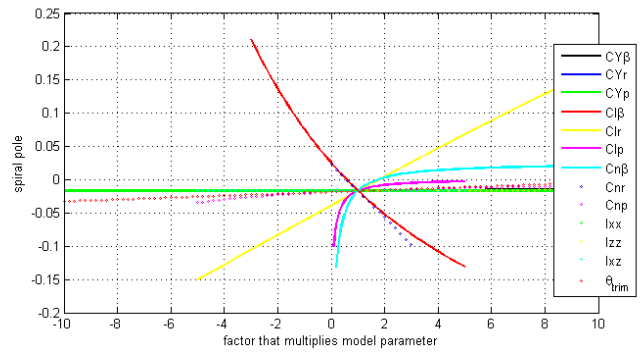

(a) Aircraft 1

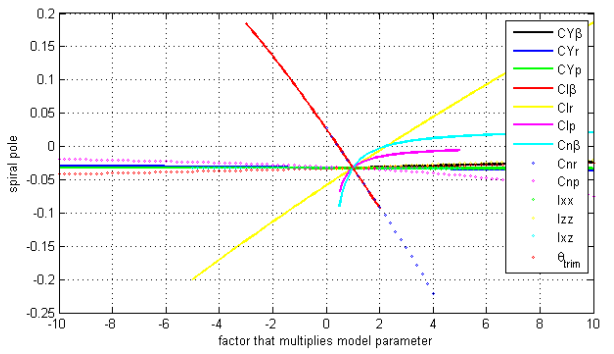

(b) Aircraft 2

Figure E.6: Spiral pole

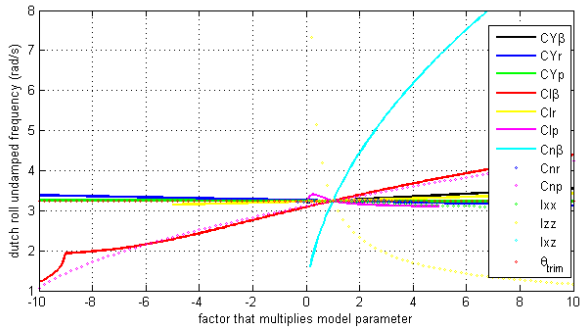

(a) Aircraft 1

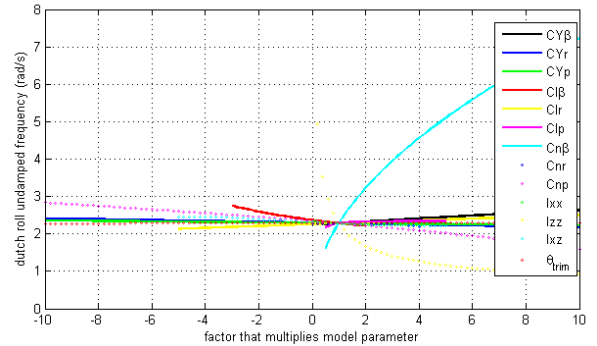

(b) Aircraft 2

Figure E.7: Dutch roll undamped frequency 


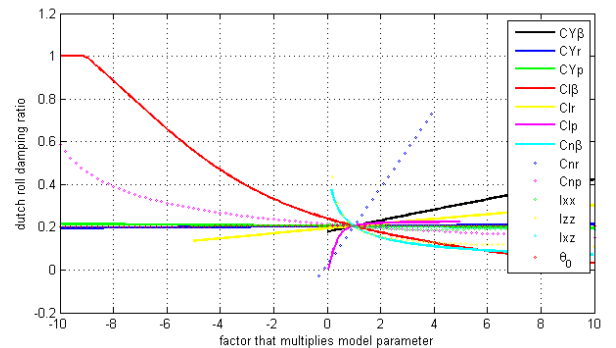

(a) Aircraft 1

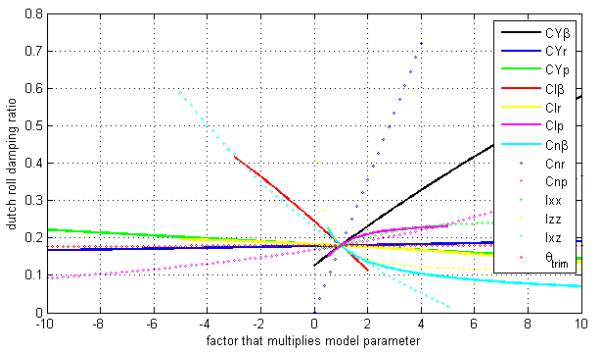

(b) Aircraft 2

Figure E.8: Dutch roll damping ratio 



\section{Appendix F}

\section{Effect of Design on Model}

\section{Parameters}

\section{Longitudinal Model Parameters}

\begin{tabular}{|c|c|c|c|c|}
\hline \multirow{2}{*}{ D. Param. } & \multicolumn{2}{|c|}{$a=0.2$} & \multicolumn{2}{|c|}{$a=-0.2$} \\
\hline & Long. Derivatives & Mass Properties & Long. Derivatives & Mass Properties \\
\hline 1 & $\begin{aligned} \delta C_{L_{\alpha}} & =1.77 \\
\delta C_{m_{\alpha}} & =18.58 \\
\delta C_{m_{q}} & =26.89 \\
\delta C_{m_{\dot{\alpha}}} & =29.58 \\
\delta C_{L_{\delta_{e}}} & =29.44 \\
\delta C_{m_{\delta_{e}}} & =29.83 \\
\delta C_{m_{0}} & =-17.10\end{aligned}$ & $\delta I_{y y}=2.58$ & $\begin{aligned} \delta C_{L_{\alpha}} & =-1.69 \\
\delta C_{m_{\alpha}} & =-17.79 \\
\delta C_{m_{q}} & =-25.75 \\
\delta C_{L_{\delta_{e}}} & =-28.18 \\
\delta C_{m_{\delta_{e}}} & =-28.56 \\
\delta C_{m_{0}} & =16.38\end{aligned}$ & $\delta I_{y y}=-2.58$ \\
\hline 2 & $\begin{aligned} \delta C_{L_{\alpha}} & =0.19 \\
\delta C_{m_{\alpha}} & =14.89 \\
\delta C_{m_{q}} & =39.79 \\
\delta C_{m_{\dot{\alpha}}} & =33.78 \\
\delta C_{m_{\delta_{e}}} & =20.06 \\
\delta C_{m_{0}} & =-11.50\end{aligned}$ & $\delta I_{y y}=13.74$ & $\begin{aligned} \delta C_{L_{\alpha}} & =-0.25 \\
\delta C_{m_{\alpha}} & =-14.61 \\
\delta C_{m_{q}} & =-32.55 \\
\delta C_{m_{\dot{\alpha}}} & =-29.93 \\
\delta C_{m_{\delta_{e}}} & =-20.06 \\
\delta C_{m_{0}} & =11.50\end{aligned}$ & $\delta I_{y y}=-11.03$ \\
\hline 3 & $\begin{aligned} \delta C_{L_{\delta_{e}}} & =8.41 \\
\delta C_{m_{\delta_{e}}} & =8.52\end{aligned}$ & & $\begin{aligned} \delta C_{L_{\delta_{e}}} & =-9.66 \\
\delta C_{m_{\delta_{e}}} & =-9.79\end{aligned}$ & \\
\hline 8 & $\begin{aligned} \delta C_{L_{\alpha}} & =0.11 \\
\delta C_{m_{\alpha}} & =1.19 \\
\delta C_{m_{\dot{\alpha}}} & =-4.14\end{aligned}$ & $\delta I_{y y}=0.11$ & $\begin{aligned} \delta C_{L_{\alpha}} & =-0.11 \\
\delta C_{m_{\alpha}} & =-1.20 \\
\delta C_{m_{\dot{\alpha}}} & =4.17\end{aligned}$ & $\delta I_{y y}=11$ \\
\hline
\end{tabular}


Lateral Model Parameters

\begin{tabular}{|c|c|c|c|c|}
\hline \multirow{2}{*}{ D. Param. } & \multicolumn{2}{|c|}{$a=0.2$} & \multicolumn{2}{|c|}{$a=-0.2$} \\
\hline & Lat. Derivatives & Mass Properties & Lat. Derivatives & Mass Properties \\
\hline 1 & $\delta C_{l_{p}}=0.16$ & $\begin{array}{l}\delta I_{x x}=0.77 \\
\delta I_{z z}=1.39\end{array}$ & $\delta C_{l_{p}}=-0.10$ & $\begin{aligned} \delta I_{x x} & =-0.65 \\
\delta I_{z z} & =-1.30\end{aligned}$ \\
\hline 2 & $\begin{aligned} \delta C_{n_{\beta}} & =31.89 \\
\delta C_{l_{r}} & =3.80 \\
\delta C_{n_{r}} & =44.51 \\
\delta C_{n_{\delta_{r}}} & =20.02\end{aligned}$ & $\begin{array}{l}\delta I_{x x}=0.44 \\
\delta I_{z z}=4.86\end{array}$ & $\begin{array}{c}\delta C_{n_{\beta}}=-31.89 \\
\delta C_{l_{r}}=-3.80 \\
\delta C_{n_{r}}=-36.41 \\
\delta C_{n_{\delta_{r}}}=-20.02\end{array}$ & $\begin{array}{l}\delta I_{x x}=-0.44 \\
\delta I_{z z}=-3.95\end{array}$ \\
\hline 4 & $\delta C_{l_{\delta_{a}}}=8.93$ & & $\delta C_{l_{\delta_{a}}}=-10.41$ & \\
\hline 5 & $\delta C_{l_{\delta_{a}}}=22.76$ & & $\delta C_{l_{\delta_{a}}}=-20.87$ & \\
\hline 6 & $\begin{aligned} \delta C_{Y_{\beta}} & =18.02 \\
\delta C_{l_{\beta}} & =9.35 \\
\delta C_{n_{\beta}} & =52.73 \\
\delta C_{l_{p}} & =0.0771 \\
\delta C_{l_{r}} & =6.28 \\
\delta C_{n_{r}} & =33.45 \\
\delta C_{Y_{\delta_{r}}} & =36.21 \\
\delta C_{l_{\delta_{r}}} & =36.38 \\
\delta C_{n_{\delta_{r}}} & =36.24\end{aligned}$ & $\begin{array}{l}\delta I_{x x}=0.19 \\
\delta I_{z z}=0.80\end{array}$ & $\begin{array}{c}\delta C_{Y_{\beta}}=-16.63 \\
\delta C_{l_{\beta}}=-8.63 \\
\delta C_{n_{\beta}}=-48.65 \\
\delta C_{l_{p}}=-0.07 \\
\delta C_{l_{r}}=-5.79 \\
\delta C_{n_{r}}=-30.86 \\
\delta C_{Y_{\delta_{r}}}=-32.54 \\
\delta C_{l_{\delta_{r}}}=-32.69 \\
\delta C_{n_{\delta_{r}}}=-32.56\end{array}$ & $\begin{aligned} \delta I_{x x} & =-0.18 \\
\delta I_{z z} & =-0.80\end{aligned}$ \\
\hline 7 & $\begin{aligned} \delta C_{Y_{\delta_{r}}} & =6.74 \\
\delta C_{l_{\delta_{r}}} & =6.77 \\
\delta C_{n_{\delta_{r}}} & =6.75\end{aligned}$ & & $\begin{aligned} \delta C_{Y_{\delta_{r}}} & =-9.12 \\
\delta C_{l_{\delta_{r}}} & =-9.16 \\
\delta C_{n_{\delta_{r}}} & =-9.12\end{aligned}$ & \\
\hline 8 & & $\delta I_{x x}=0.05$ & & $\delta I_{x x}=-0.04$ \\
\hline
\end{tabular}




\section{Bibliography}

[1] Ferdinad P. Beer, E. Russell Johnston Jr., and Elliot R. Eisenberg. Vector Mechanics for Engineers: Statics. McGraw Hill, 8th edition, 2007.

[2] Helena V. de Castro. Flying and Handling Qualities of a Fly-by-Wire Blended-Wing-Body Civil Transport Aircraft. PhD thesis, Cranfield University, 2003.

[3] Richard Colgren and Lance Holly. Flight dynamic requirements for uavs - do they really exist? In Proceedings of 27th Atmospheric Flight Mechanics Conference and Exhibit, Chicago, August 2009. AIAA.

[4] MV Cook. Flight Dynamics Principles. Butterworth-Heinemann, 2007.

[5] Christopher M. Cotting. Applicability of Human Flying Qualities Requirements for UAVs, Finding a Way Forward. In Proceedings of 27th Atmospheric Flight Mechanics Conference and Exhibit, Chicago, August 2009. AIAA.

[6] Daniel Coutinho. Control System Integration in a UAV. Master's thesis, Academia da Força Aérea/Instituto Superior Técnico, 2008.

[7] B. Etkin and L.D. Reid. Dynamics of flight- Stability and control. 1996.

[8] European Aviation Safety Agency,. Certification Specifications for Normal, Utility, Aerobatic, and Commuter Category Aeroplanes CS-23, November 2003.

[9] L. Félix and D. Saraiva. Determinação das Derivadas de Estabilidade e Momentos de Inércia do UAV Antex-M X03. Master's thesis, Academia da Força Aérea, 2007.

[10] R. D. Fink. USAF Stability and Control DATCOM. McDonnell Douglas Corporation, 1978.

[11] Tyler M. Foster. Dynamic Stability and Handling Qualities of Small Unmanned Air Vehicles. Master's thesis, Brigham Young University, 2005.

[12] J. Hodgkinson. Aircraft Handling Qualities. Blackwell Science, 1999. 
[13] Jessica A. Holmberg, Daniel J. King, John R. Leonard, and Christopher M. Cotting. Flying qualities specifications and design standards for unmanned air vehicles. In Proceedings of 26th Atmospheric Flight Mechanics Conference and Exhibit, Hawaii, August 2008. AIAA.

[14] Military Standard, Flying Qualities of Piloted Aircraft MIL-STD 1797A. US Department of Defense, 1990.

[15] Military Specification, Flying Qualities of Piloted Airplanes, MIL-F-8785C , August 1996.

[16] Jan Roskam. Airplane Flight Dynamics and Automatic Flight Controls, volume 1. Design, Analysis and Reserach Corporation (DARCorporation), 2001.

[17] Jan Roskam and Chuan-Tau E. Lan. Airplane aerodynamics and performance. Design, Analysis and Reserach Corporation (DARCorporation), 1997.

[18] B. L. Stevens and F. L. Lewis. Aircraft Control and Simulation. John Wiley \& Sons, Inc., New York, 2nd edition, 2003.

[19] U.S. Naval Test Pilot School. Flight Test Manual: Fixed Wing Stability and Control - Theory and Flight Test Techniques, 1997.

[20] Warren Williams. UAV Handling Qualities.....You Must be Joking. 2003.

[21] Peter H. Zipfel. Modeling and Simulation of Aerospace Vehicle Dynamics. AIAA Education Series, 2007. 